NASA Contractor Report 3744

\title{
Rotor-Vortex Interaction Noise
}

\author{
Robert H. Schlinker and Roy K. Amiet
}

CONTRACT NAS1-16392

OCTOBER 1983

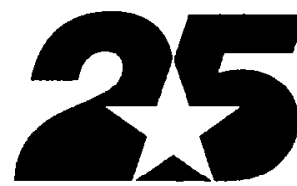

25th Anniversary

1958-1983

\section{N/Sก}

LOAN COPY: RETURN TO AFWL TECHNICAL LIBRARY KIRTLAND AFB, N.M. 87117 
NASA Contractor Report 3744

\section{Rotor-Vortex Interaction Noise}

Robert H. Schlinker and Roy K. Amiet

United Technologies Research Center

East Hartford, Connecticut

Prepared for

Langley Research Center

under Contract NAS1-16392 
. 


\section{Rotor-Vortex Interaction Noise}

TABLE OF CONTENTS

$\underline{\text { Page }}$

SUMMARY. . . . . . . . . . . . . . . . . . . . . . . . 1

INTRODUCTION . . . . . . . . . . . . . . . . . . . 2

Background. . . . . . . . . . . . . . . . . . . . 2

Previous Investigations . . . . . . . . . . . . . . 3

Present Investigation .. . . . . . . . . . . . . . . 4

LIST OF SYMBLS. . . . . . . . . . . . . . . . . . . 6

ANALYTICAL FORMULATION OF THE BLADE VORTEX INTERACTION MECHANISM . .10

Definition of the Problem and Description of Approach . . . . . 10

General Expression for Far-Field Sound. . . . . . . . . . . 11

The Airfoil Lift Response . . . . . . . . . . . . . . 13

The Velocity Upwash . . . . . . . . . . . . . . . . . 16

The Velocity Upwash for an Arbitrary Vortex Orientation . . . . 18

Application of the Theory . . . . . . . . . . . . . . 19

DESCRIPTION OF THE EXPERIMENT. . . . . . . . . . . . . . . 21

Acoustic Research Tunnel. . . . . . . . . . . . . 21

Model Rotor. . . . . . . . . . . . . . . . 22

Instrumentation . . . . . . . . . . . . . . . . . 23

Test Program. . . . . . . . . . . . . . . . . . 26

Qualification of Acoustic Test Procedure. . . . . . . . . 29

VELOCITY FIELD INCIDENT ON THE ROTOR . . . . . . . . . . . . . . 31

Objective . . . . . . . . . . . . . . . . . 31

Approach. . . . . . . . . . . . . . . . . . . . . 32

Vortex Azimuthal and Axial liean Velocity Fleld. . . . . . . 32

Axial Velocity Defect in Two Dimensional Wake . . . . . . . 34

Turbulence in Viscous Core. . . . . . . . . . . . . . 35

Changes in Velocity Field Due to Contraction of Flow. . . . . 35

Unsteady Upwash Due to Blade Vortex Interaction . . . . . . . 37 
TABLE OF CONTENTS (Cont'd)

$\underline{\text { Page }}$

EXPERIMENTAL ASSESSMENT OF BLADE VORTEX INTERACTION NOISE $\cdot \ldots . .39$

Objective. . . . . . . . . . . . . . . . . . . 39

Effect of Blade-Vortex Interaction . . . . . . . . . . . . 39

Blade Number Dependence. . . . . . . . . . . . . . 43

Vortex Strength Dependence . . . . . . . . . . . . . . . . 44

Vortex Intersection Angle Dependence . . . . . . . . . . . . 45

Blade Pitch Angle Dependence . . . . . . . . . . . . . . 47

Mach Number Dependence . . . . . . . . . . . . . . 48

Directivity Pattern. . . . . . . . . . . . . . . . . . 48

Assessment of Acoustic Spectrum and Pressure Signature . . . . . 50

CONCLUSIONS ............................ 53

APPENDIX A - Vortex Velocity Field Measurement Technique. . . . . . 54

APPENDIX B - Experimental Technique for Isolating Blade Vortex Interaction Impulsive Signatures ........ 56

REFERENCES. ........................ 57

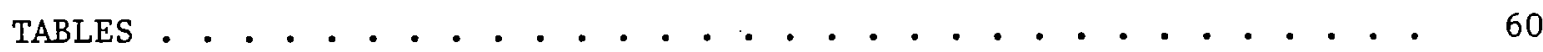

FIGURES. ......................... 61 


\section{SUMPARY}

A theoretical and experimental study was conducted to develop a validated first principles analysis for predicting noise generated by helicopter main-rotor shed vortices interacting with the tail rotor. A generalized noise prediction procedure was formulated in which the incident vortex velocity field, rotor geometry, and rotor operating conditions are input variables. The analysis includes compressibility effects, chordwise and spanwise noncompactness, and treats oblique intersections with the blade planform. Output from the analysis provides acoustic spectra and pressure signatures as a function of observer position.

Assessment of the theory involved conducting a model rotor experiment which isolated the blade-vortex interaction noise from other rotor noise mechanisms. The experiment was conducted with a $0.76 \mathrm{~m}$ articulated rotor operating in the United Technologies Research Center Acoustic Research Tunnel. An isolated tip vortex, generated by an upstream semispan airfoil, was convected into the model rotor to generate blade-vortex interaction noise. Acoustic spectra, pressure signatures, and directivity were measured for different combinations of rotor tip Mach number, blade pitch, and blade number. Since input to the acoustic prediction required a knowledge of the vortex properties, blade vortex intersection angle and intersection station were determined using smoke visualization. Vortex strength and vortex core radius were documented with hot film probe measurements.

Ingestion of the vortex by the rotor was experimentally observed to generate harmonic noise and impulsive waveforms. Assessment of the theory using the measured data verified the linear dependence on blade number and vortex strength. The absence of noise for vortex trajectories normal to the blade was also predicted. For oblique intersections with a blade, changes in acoustic radiation, due to intersection angle modifications, were calculated to within 2 decibels of the measured data. A simplified scaling law was also developed to predict these changes. Low sensitivity to blade pitch angle modifications for typical rotor operating conditions was confirmed experimentally. The strong dependence on local Mach number was predicted although the sensitivity to Mach number changes was overpredicted at low frequencies.

Measured blade vortex interaction directivity patterns confirmed the theoretical dipole acoustic source model including the location of the minimum in the plane of the blade. Absolute levels for acoustic spectra and directivity patterns were calculated, without the use of empirical or adjustable constants, to within 5 decibels at low frequencies. At high frequencies, sound pressure levels were underpredicted. General features of the measured vortex interaction acoustic pressure signature were calculated but the exact waveform shape and amplitude were not predicted. 


\section{INTRODUCTION}

\section{Background}

The noise level at a given observer position relative to a helicopter and within a specified frequency band represents the combined effect of a number of separate acoustic source mechanisms. Some mechanisms occur only on the main rotor, others apply to both the main rotor and tail rotor, and in more complex situations, interactions occur linking the wakes of one rotor with noise generated by the other rotor. The simultaneous occurrence of the acoustic source mechanisms has complicated the design of quiet helicopters. and contributed to empiricism and extrapolation of measured data when predicting full scale helicopter noise signatures.

Due to the limitations of empirically based helicopter noise prediction procedures, an increasingly important objective of helicopter noise research has been the development of procedures based on first principles analyses. This effort has been reasonably successful for a number of noise mechanisms such as blade thickness, loading, turbulence ingestion, and boundary layertrailing edge interaction. However, in the area of main-rotor shed vortices interacting with the tail rotor, first principle analyses did not exist. The lack of a firm understanding of the tail-rotor vortex interaction mechanism created a need for an experimentally validated prediction procedure. In an effort to achieve this objective, the present joint experimental and theoretical research program was conducted to assess this noise mechanism.

The blade-vortex interaction mechanism addressed here is considered to be potentially important. As illustrated in Figure la, under forward flight or vertical descent operating conditions vortices shed by the main rotor pass through the tail rotor resulting in the generation of noise. In principle, the strength of this noise mechanism can be reduced by changes in the helicopter design. For example, altering the relative position of the main rotor and tail rotor is a possible solution. In addition, changing the main-rotor blade tip shape may reduce the strength of the tip vortex or diffuse the vortex velocity field in a manner which reduces interaction noise.

The decision to pursue such aerodynamic design changes is based on the ability to predict quantitatively the expected benefits. The major objective of the present study was, therefore, to develop and validate a first principles prediction procedure which starts with the incident vortex velocity field as an input. 


\section{Previous Investigations}

Experimental Studies - Few experimental studies of helicopter tail-rotor vortex ingestion noise are currently available. Leverton, et al. (ref. 1) observed that the Lynx helicopter produced such noise during approach conditions when the tail rotor intersected tip vortices shed by the main rotor. The experimental results indicated that this noise mechanism can be subjectively annoying and can dominate over the main rotor and engine noise.

In addition to full scale measurements, Pegg and Shidler (ref. 2) studied the parameters controlling main-rotor tail-rotor interaction noise using a small scale helicopter model. The model permitted varying tail rotor position relative to the main rotor in addition to the tail rotor speed and rotation direction. Experimental results showed that certain features of the tail rotor acoustic spectrum were attributable to interactions with tip vortices shed from the main rotor.

A limited study of model scale vortex interaction noise was also conducted by Paterson and Amiet (ref. 3). Interaction of an isolated tip vortex with the model rotor was observed to produce significant harmonic noise which extended to high frequency. The impulsive acoustic waveform generated by the interaction was found to be sensitive to vortex strength and position.

Theoretical Studies - Analytical models predicting the blade vortex interaction noise mechanism presently treat only the main rotor noise source. To help differentiate these analyses from the present formulation, a brief discussion of the main rotor interaction mechanism will be given. In this case the blade and vortex lie in different (or identical) parallel planes. Furthermore, the blade leading edge is parallel to the vortex centerline in the most intense interactions (Fig. Ib). This condition is modeled by a two dimensional analysis assuming end effects can be neglected. On the other hand, if the vortex centerline is skewed relative to the airfoil leading edge (but still parallel to rotor plane), the vortex velocity field sweeps across the airfoil. Under this condition, a three dimensional analysis is required.

The above described two dimensional blade vortex interaction was treated by Amiet (ref. 4) assuming linearized flow. The three dimensional interaction was modeled by Widnall (ref. 5), Widnall and Wolf (ref. 6) and Adamczyk (ref. 7). These analyses include the two dimensional problem as a special case but they do not treat the present tail rotor interaction geometry which involves a nonplanar intersection. In this case, the rotor disk is approximately at $90^{\circ}$ to the vortex plane at the intersection station. One objective of the present study was, therefore, to develop the specific analyses needed to treat the tail rotor blade vortex interaction geometry. 
Objective - The overall objective of the present study was to develop an experimentally validated blade-vortex interaction analysis. The analytical phase of the program required the formulation of a generalized noise prediction procedure in which the incident vortex velocity field, rotor geometry, rotor operating conditions, and far field observer position were input variables. Empirical scaling factors were avoided in this first principles analysis.

Assessment of the theory required conducting a tractable acoustic experiment which isolated this noise mechanism from other operative rotor noise mechanisms. Also, detailed measurements of the incident vortex velocity field were obtained to provide the experimental input to the analysis. Confirmation of the theory for the experimental test conditions involved assessment of blade vortex interaction noise sensitivity to changes in the vortex field and the rotor operating conditions.

Theoretical Approach - The present analysis transforms the incident vortex velocity field into a spectrum of sinusoidal gusts incident on an isolated rotor blade. Linearized theory is then used to determine the unsteady blade lift response function. The response is given by a generalized function which includes compressibility effects, chordwise and spanwise noncompactness, and treats skewed or normal gusts in the decomposition of the incident vortex field. Input to the analysis requires a knowledge of the vortex strength, vortex viscous core radius, blade-vortex intersection angle, blade vortex intersection station, blade tip Mach number, blade pitch angle, blade chord, blade number, and far field observer location. Output from the analysis provides acoustic spectra and pressure signatures as a function of observer position.

Experimental Approach - Figures 2 and 3 show the experimental arrangement employed to assess the present analysis. Here a tip vortex, generated by an upstream semispan airfoil model, is convected into a model helicopter rotor operating in vertical ascent with a $9 \mathrm{~m} / \mathrm{sec}$ inflow velocity. Although this test configuration lacks the appearance of a tail rotor, it represents a tractable geometry which permits direct assessment of the blade-vortex interaction mechanism. Selection of this geometry was based on an evaluation of the parameters controlling the noise mechanism and a detailed discussion is included in the description of the experiment.

The experiment was conducted with a $0.76 \mathrm{~m}$ diameter articulated rotor. Acoustic spectra, pressure signatures and directivity were measured for different combinations of rotor tip Mach number, blade pitch, and blade number. Blade vortex intersection angle and blade intersection station were documented 
with smoke visualization. Vortex strength and core radius in addition to viscous core turbulence intensity and length scale were documented with hot film probe measurements.

It was considered important to devise an experiment in which only one parameter varied at a time. This was necessary since the vortex and rotor aerodynamic parameters were coupled. For example, changing the rotor tip Mach number also changed the mean flow contraction ratio for the rotor orientation shown in Figure 2. Hence, the incident vortex strength, intersection angle, and intersection station also changed. Selection of the test matrix, therefore, required documentation of these coupling effects. 
c

$c_{0}$

$c_{2}$

D

E*

$\mathrm{F}$

f

$f(M)$

g

$G, G_{\infty}$

$\mathrm{H}$

h

I

$i$

$\mathrm{J}_{\mathrm{n}} \mathrm{Y}$

$\mathrm{J}_{\mathrm{ux}}$

K

$\mathrm{K}_{1}$ angle between hot film sensor and total velocity vector semichord

rotor blade number

blade passing frequency

chord

sound speed

upstream semispan airfoil chord length, $11.4 \mathrm{~cm}$

vortex core diameter

combination of Fresnel integrals

point force

frequency, cycles/sec

function of Mach number; see Eq. (13)

normalized pressure jump; see Eq. (2)

single sided spectrum for far field sound generated by single and multiple vortex intersections

vertical separation distance between tip of semispan airfoil and tip of rotor blade

semispan airfoil open jet penetration height

distance from rotor blade tip to vortex intersection station $\sqrt{-1}$

Bessel functions of the first and second kind

Eulerian time scale in autocorrelation

coefficient relating velocity and voltage for hot film probe measurements

vortex strength 


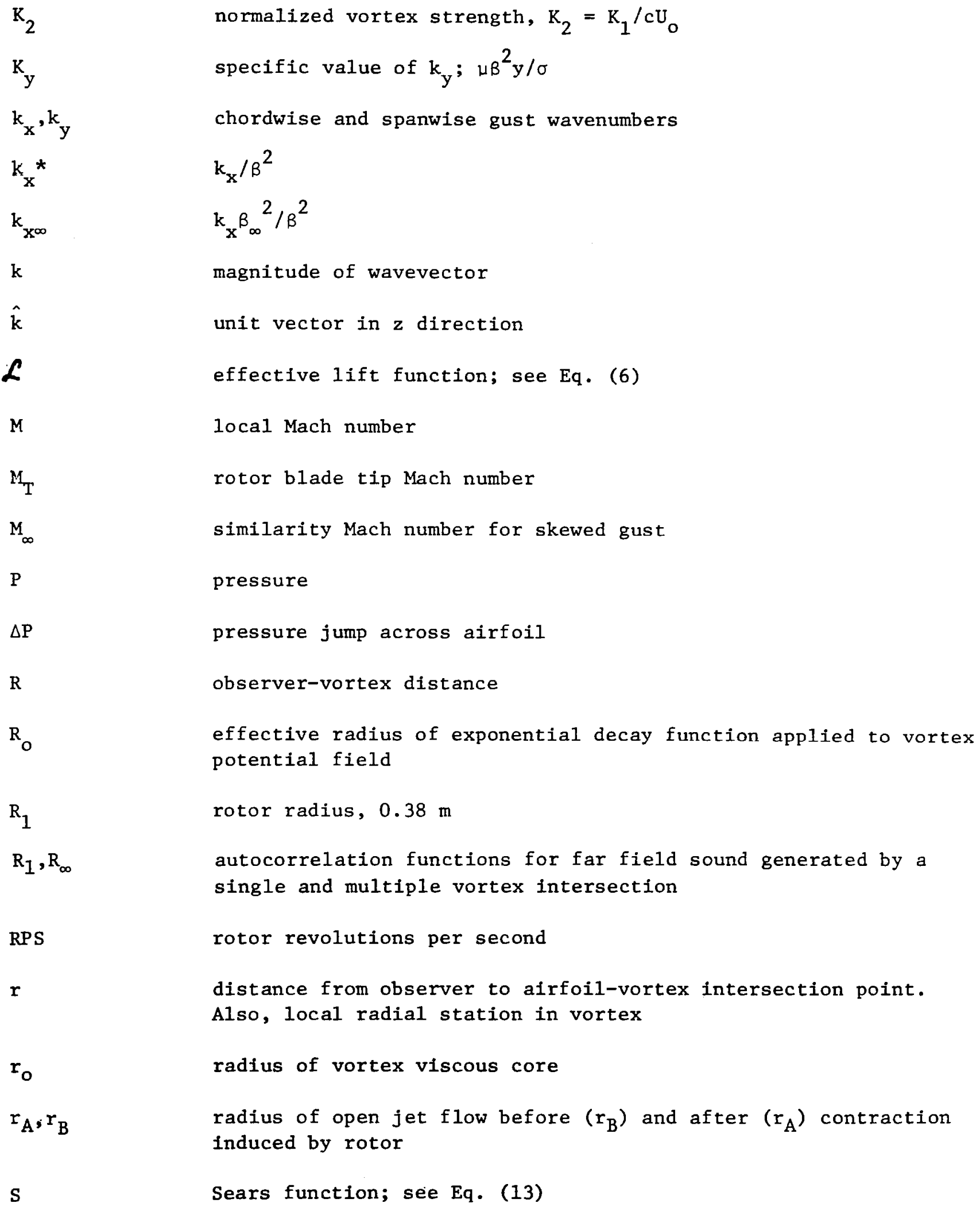




\begin{tabular}{|c|c|}
\hline $\mathrm{s}_{1}, \mathrm{~s}_{\propto}$ & $\begin{array}{l}\text { two sided spectrum for far field sound generated by a single } \\
\text { and multiple vortex intersection }\end{array}$ \\
\hline SPL & sound pressure level in $\mathrm{dB}$ relative to 0.0002 dyne $/ \mathrm{cm}^{2}$ \\
\hline $\mathrm{T}$ & time increment in Fourier summation \\
\hline $\mathrm{t}$ & time \\
\hline $\mathrm{U}$ & $\begin{array}{l}\text { fluid velocity relative to airfoil in present theory; } c_{0}{ }^{M} \text {. } \\
\text { Also, axial velocity in vortex core or in semispan airfoil two } \\
\text { dimensional wake }\end{array}$ \\
\hline $\mathrm{U}_{\mathrm{N} 1}, \mathrm{U}_{\mathrm{N} 2}$ & $\begin{array}{l}\text { velocity components normal to hot film probe sensing element in } \\
\text { two different orientations }\end{array}$ \\
\hline $\mathrm{U}_{\mathrm{T}}$ & total velocity vector in the plane of the hot film sensor \\
\hline V & sweep speed of gust along airfoil relative to airfoil \\
\hline$v_{1}, v_{2}, v_{3}, v_{4}$ & vortex azimuthal velocity vectors \\
\hline $\mathrm{V}_{\mathrm{N}}$ & vortex velocity component normal to blade planform \\
\hline $\mathrm{V}_{\mathrm{r}}$ & sweep speed of gust along airfoil relative to fluid \\
\hline $\mathrm{V}_{\theta}$ & vortex azimuthal velocity \\
\hline$v_{0}$ & maximum vortex velocity \\
\hline $\mathrm{w}_{\mathrm{g}}$ & sinusoidal gust velocity normal to plane of airfoil \\
\hline$\tilde{\tilde{\mathbf{w}}}$ & Fourier amplitude of $\mathrm{w}_{\mathrm{g}}$ \\
\hline $\mathrm{x}, \mathrm{y}, \mathrm{z}$ & Cartesian coordinates; see Fig. 4 \\
\hline $\mathrm{x}_{1}, \mathrm{y}_{1}$ & airfoil coordinates \\
\hline$\alpha$ & angle between gust and airfoil. Also, constant used in Eq. (22) \\
\hline$\alpha_{1}$ & rotor blade pitch angle \\
\hline$\alpha_{2}$ & semispan airfoil angle of attack \\
\hline$B$ & $\sqrt{1-M^{2}}$ \\
\hline
\end{tabular}




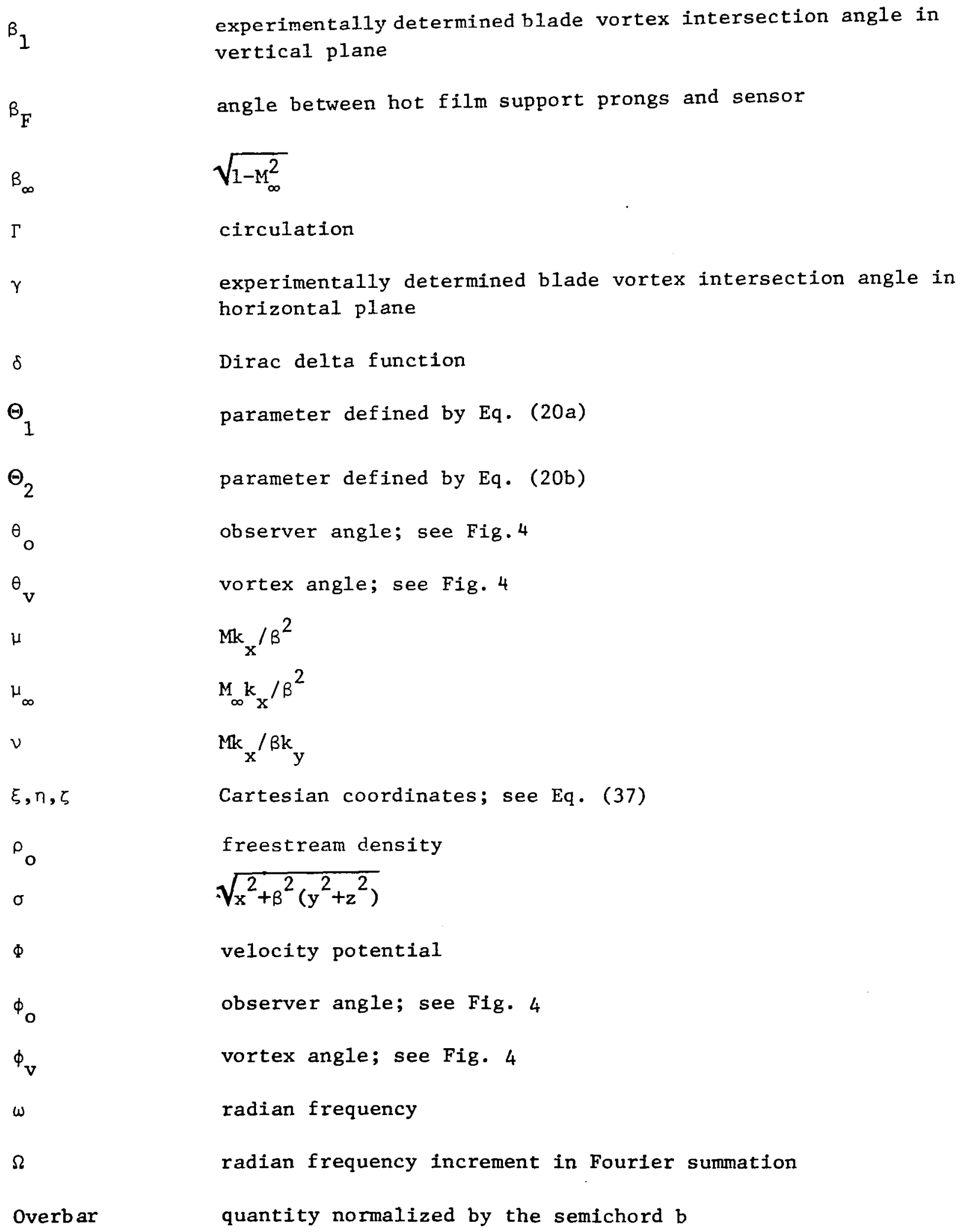


ANALYTICAL FORMULATION OF THE BLADE VORTEX INTERACTION MECHANISM

Definition of the Problem and Description of Approach

The present analysis was developed for a blade vortex interaction geometry in which the rotor plane of rotation and the vortex plane are skewed. Consequently, the blade cuts through the vortex centerline during Its motion as illustrated by the combined airfoil and vortex geometry shown in Figure 4. The orientation of the vortex relative to the airfoil is arbitrary. Small interaction angles between the airfoil plane and the vortex centerline are, however, not permitted in the analysis. This restriction occurs because the intersection point of the airfoil and the vortex is assumed to be localized on the airfoil allowing end effects to be neglected. Thus, the blade vortex orientation treated in references $1-4$ is excluded from the present analysis.

The present formulation is based on linearized theory with the rotor blade modeled as a flat plate of infinite span. Because of the linearized flow assumption, steady loading is assumed to be small and is not coupled with the unsteady blade vortex interaction noise mechanism. Standard airfoil theory dictates that only the fluid velocity normal to the airfoil plane affects the airfoil loading. Also, because of the linearized flow assumption, the vortex velocity field and its trajectory are not altered by velocity perturbations induced by the airfoil.

Analytical expressions used for the airfoil response functions are for the case of a flat plate airfoil of infinite span. A sinusoidal gust upwash is assumed incident on the airfoil. Thus, the airfoil response functions are similar to the classical sears function (ref. 8). The only difference between the airfoil response functions used here and the Sears function is that the present solutions extend the Sears solution to the case of compressible flow. Also, using the similarity rule of Graham (ref. 9), the gust can be skewed relative to the airfoil. If the Mach number is set equal to zero and the gust is assumed parallel to the airfoil, the present solution reduces exactly to that of Sears.

A detailed derivation of the present gust response functions is given in references 10-14. Separate solutions are given for the low and high frequency regime. The low frequency solution is a series type of solution and the high frequency solution is based on a convergent iteration procedure; the solution produced is asymptotically correct as frequency goes to infinity. Comparison with exact numerical solutions of Graham (refs. 9, 15) shows that the present solutions give an error in the lift of at most a few percent. The maximum error occurs near the changeover point from the low to the high frequency solutions. 
The model for the vortex velocity field is the same one used by several other authors (refs. 16, 17). It reduces to a potential vortex at large distance and approaches a solid body rotation in the core. This avoids the infinite velocity existing at $r=0$ in a potential vortex.

\section{General Expression For Far-Field Sound}

The response of the airfoil to the vortex velocity field will be treated by Fourier decomposing the velocity field and calculating the airfoil response to each resulting gust component. The inverse Fourier transform then gives the time behavior of the gust-vortex interaction.

The airfoil is assumed to be a flat plate of infinite span lying in the $x, y$ plane, with the leading edge at $x=-b$ (see Fig. 1). If the airfoil lift were being calculated, the assumption of infinite span would create problems; since the vortex field has only an $\mathrm{r}^{-1}$ decay rate, an infinite lift would be obtained in general. This singularity would become apparent on attempting to perform the Fourier inversion of the expression for lift produced by a gust component; this has $a^{-1}$ wavenumber behavior for small $k$. However, since the present analysis calculates the sound and the sound is dependent on the time derivative of the loading, an additional factor of $k$ is introduced which eliminates the singularity at $k=0$. There remains, however, a difficulty in the calculated value of the average pressure level. This is considered in the section titled Experimental Assessment of Blade Vortex Interaction Noise.

Linearized flow is assumed. This means that the vortex remains fixed relative to the mean flow at infinity. Thus, on Fourier decomposition the $z$ component of the vortex velocity field in the $x, y$ plane can be written in terms of the gusts

$$
w_{g}=\widetilde{w}\left(k_{x}, k_{y}\right) e^{-i\left[k_{x}(x-U i)+k_{y} y\right]}
$$

This gust will lead to a pressure jump across the airfoil which can be written

$$
\Delta P\left(x_{1}, y_{1}, k_{x}, k_{y}\right)=2 \pi \rho_{0} u \widetilde{\widetilde{w}}\left(k_{x}, k_{y}\right) g\left(x_{1}, k_{x}, k_{y}\right) e^{-i\left(k_{y} y_{1}-k_{x}(t)\right.}
$$

The far-field pressure of a tranverse point dipole or force $F \hat{k} e^{i \omega t}$ at $\mathrm{x}_{1}, \mathrm{y}_{1}$ is

$$
P_{F}=\frac{i F \omega z}{4 \pi C_{0} \sigma^{2}} e^{i[\omega t+\mu(M x-\sigma)]} e^{-i \mu\left[M x_{1}-\left(x x_{1}+\beta^{2} y y_{1}\right) / \sigma\right]}
$$


This is obtained from the usual expression for a dipole (ref. 11) by neglecting terms $O\left(r_{1} / r\right.$ ) where $r_{1}$ is a source (airfoil) dimension and $r$ is the source-observer distance.

The far-field pressure due to the gust component $w_{g}$ is found by replacing $F$ in Equation 3 with $\Delta \mathrm{P}$ from Equation 2 noting that

$$
\boldsymbol{\omega}=k_{\mathbf{x}} U
$$

and integrating over the airfoil surface. This gives

$$
\widetilde{P}\left(k_{x}, k_{y}\right)=\frac{i \pi \rho_{0} U^{2} b k_{x} z}{c_{0} \sigma^{2}} \delta\left(k_{y}-\mu \beta^{2} y / \sigma\right) \widetilde{w}\left(k_{x}, k_{y}\right) \mathcal{L}\left(k_{x}, k_{y}, M\right) e^{i\left[k_{x} U t+\mu(M x-\sigma)\right](5)}
$$

where

$$
\mathcal{L}\left(k_{x}, k_{y}, M\right) \equiv \frac{1}{b} \int_{-b}^{b} g\left(x_{1}, k_{x}, k_{y} M\right) e^{-i \mu(M-x / \sigma) x_{1}} d x_{1}
$$

represents an effective lift per unit span. The exponential phase factor in $\mathcal{L}$ accounts for retarded time effects for sound propagating from source to observer; if this factor were set equal to $1, \mathcal{L}$ would represent the actual lift per unit span.

To find the far-field pressure-time response, the integral over all $\mathrm{k}_{\mathrm{x}}$ and $k_{y}$ gust components is taken giving

$$
P(t)=-\pi \frac{b z \rho_{0} U^{2}}{c_{0} \sigma^{2}} \int_{-\infty}^{\infty}\left[-i k_{x} \approx\left(k_{x}, k_{y}\right)\right] \mathcal{L}\left(k_{x}, k_{y}, M\right) e^{i\left[k_{x} U \dagger+\mu(M x-\sigma)\right]} d k_{x}(7)
$$

where

$$
\begin{aligned}
k_{y} & \equiv \mu \beta^{2} y / \sigma \\
\mu & \equiv k_{x} M / \beta^{2} \\
\beta^{2} & \equiv 1-M^{2} \\
\sigma^{2} & \equiv x^{2}+\beta^{2}\left(y^{2}+z^{2}\right)
\end{aligned}
$$

The factor $\exp [i \mu(M x-\sigma)]$ gives only a time shift $\Delta t=(\sigma-M x) / \beta^{2} c_{0}$ to account for the time for the sound to propagate from the airfoil leading edge to the far field and can be ignored. Thus,

$$
P(t)=-\pi \frac{b z \rho_{0} u^{2}}{c_{0} \sigma^{2}} \int_{-\infty}^{\infty}\left[-i k_{x} \approx \tilde{w}\left(k_{x}, k_{y}\right)\right] \mathcal{L}\left(k_{x}, k_{y}, M\right) e^{i k_{x} U t} d k_{x}
$$


Graham (ref. 9) has shown that for a flat plate infinite span airfoil in subsonic flow the response function to a skewed gust can be obtained by similarity considerations from either (1) the gust response of the airfoil to a parallel gust in compressible flow or (2) the gust response of the airfoil to a skewed gust in incompressible flow. The first or second solution should be used depending on whether the intersection point of the gust wavefront with the airfoil leading edge moves supersonically or subsonically relative to the fluid, respectively.

For the present case it can readily be shown that the intersection point always moves supersonically for the gusts of interest. Basically this is because a subsonic gust leads to an exponentially decaying pressure field away from the airfoil. The angle $\alpha$ of the gust wavefront relative to the airfoil leading edge is

$$
\tan \alpha=k_{y} / k_{x}=M_{y} / \sigma
$$

The intersection point sweeps along the airfoil with velocity

$$
v=U \cot \alpha=c_{0} \sigma / y
$$

Relative to the fluid the velocity is

$$
v_{r}=\sqrt{u^{2}+v^{2}}=c_{0} \sqrt{x^{2}+y^{2}+\beta^{2} z^{2} / y} \geq c_{0}
$$

Thus, the solution requires only the airfoil response to a parallel gust in subsonic flow, along with the similarity rules of Graham.

The similarity rule of Graham (ref. 9) relates the surface pressure gust response functions $g\left(\xi, k_{x}, k_{y}, M\right)$ for a skewed gust in compressible flow to the function $g\left(\xi, k_{x}, 0, M\right)$ for a parallel gust in a flow of Mach number M. The relation is

$$
g\left(\xi, k_{x}, k_{y}, M\right)=\frac{\beta_{\infty}}{\beta} g\left(\xi, k_{x}, 0, M_{\infty}\right) e^{i \xi k_{y}^{2} / k_{x}}
$$

where

$$
\begin{aligned}
& k_{x \infty} \equiv k_{x} \beta_{\infty}{ }^{2} / \beta^{2} \\
& \beta_{\infty}{ }^{2} \equiv 1-M_{\infty}^{2} \\
& M_{\infty} \equiv M \sqrt{1-\nu-2}
\end{aligned}
$$




$$
\begin{gathered}
\nu \equiv M k_{x} / \beta k_{y} \\
\mu_{\infty} \equiv M_{\infty} k_{x \infty} / \beta_{\infty}{ }^{2}=M_{\infty} k_{x} / \beta^{2}
\end{gathered}
$$

The response function for a parallel gust is divided into two ranges in which different approximate solutions are used. (ref. 10)

Case 1: $\mu_{\infty} \leq 0.4$ For low frequency an approximate gust response function is

$$
g\left(x, k_{x}, O, M\right)=\frac{1}{\pi \beta} \sqrt{\frac{1-\bar{x}}{1+\bar{x}}} S\left(\bar{k}_{x}^{*}\right) e^{i \bar{x}_{x}^{*}\left[M^{2} \bar{x}+f(M)\right]}
$$

where

$$
\begin{gathered}
k_{x}^{*} \equiv k_{x} / \beta^{2} \\
f(M) \equiv(1-\beta) \ln M+\beta \ln (1+\beta)-\ln 2
\end{gathered}
$$

and the bar over $\mathrm{x}$ and $\mathrm{k}_{\mathrm{x}}^{*}$ indicates normalization by the semichord $\mathrm{b}$. The function $S$ is the Sears function (ref. 8) used in incompressible flow airfoil theory. The above solution ignores terms $0\left(\mathrm{M}^{2} \mathrm{k}_{\mathrm{x}}^{* 2}\right)$ but retains lower order terms such as $0\left(\mathrm{k}_{\mathrm{x}}^{* 2}\right)$, $0\left(M^{2}\right), 0\left(k_{x}^{*} M^{2}\right)$. Substituting Equation 13 for $g\left(\xi, k_{x^{\infty}}, 0, M_{\infty}\right)$ in Equation 12 gives

$$
g\left(x, k_{x}, K_{y}, M\right)=\frac{1}{\pi \beta} \sqrt{\frac{1-\bar{x}}{1+\bar{x}}} s\left(\bar{k}_{x}^{*}\right) e^{i \mu M x+i \bar{k}_{x}^{*} f\left(M_{\infty}\right)}
$$

Together with Equation 6 this gives for $\mathcal{L}$

$$
\mathcal{L}\left(k_{x}, K_{y}, M\right)=\frac{1}{\beta} S\left(\bar{k}_{x}^{*}\right) e^{i \bar{k}_{x}^{*} f\left(M_{\infty}\right)}\left[J_{0}(\bar{\mu} x / \sigma)-i J_{1}(\bar{\mu} x / \sigma)\right]
$$

Case 2: $\mu_{\infty} \geq 0.4$ For high frequency the airfoil response can be calculated using a method of Landahl (ref. 19) which treats the airfoil as a semiinfinite flat plate with a leading edge but no trailing edge. A correction can be added which treats the airfoil as a semiinfinite flat plate with a trailing edge but no leading edge. An iteration can be carried out between the leading and trailing edges and was shown by Landahl to converge for all frequency. The first two terms of this solution are (refs. $7,11,12$ )

$$
g_{1}\left(x, k_{x}, O, M\right)=\frac{1}{\pi \sqrt{\pi(1+M) \vec{k}_{x}(1+\bar{x})}} e^{-i\left[\bar{\mu}(1-M)(1+\bar{x})+\pi / 4-\bar{k}_{x}\right]}
$$




$$
g_{2}\left(x, k_{x}, O, M\right) \approx \frac{1}{\pi \sqrt{2 \pi(1+M) \bar{k}_{x}}}\left[(1+i) E^{*}(2 \bar{\mu}(1-\bar{x}))-1\right] \mathrm{e}^{-i\left[\bar{\mu}(1-M)(1+\bar{x})+\pi / 4-\bar{k}_{x}\right]}
$$

where

$$
E^{*} \equiv \frac{1}{\sqrt{2 \pi}} \int_{0}^{x} e^{-i \xi} \frac{d \xi}{\sqrt{\xi}}
$$

$E^{*}$ is a combination of Fresnel integrals. Substitution in Equation 12 gives for a skewed gust

$$
\begin{aligned}
g_{1}\left(x, k_{x}, k_{y}, M\right)= & \frac{1}{\pi \sqrt{\pi \bar{k}_{x}\left(1+M_{\infty}\right)(1+\bar{x})}} e^{-i \bar{k}_{x}^{*}\left[M_{\infty}(1+\bar{x})-M^{2} \bar{x}-1\right]-i \pi / 4} \\
g_{2}\left(x, k_{x}, k_{y}, M\right) \approx & \frac{1}{\pi \sqrt{2 \pi \bar{k}_{x}\left(1+M_{\infty}\right)}} e^{-i \bar{k}_{x}^{*}\left[M_{\infty}(1+\bar{x})-M^{2} \bar{x}-1\right]-i \pi / 4} \\
& {\left[(1+i) E^{*}\left(2 \bar{k}_{x}^{*} M_{\infty}(1-\bar{x})\right)-1\right] }
\end{aligned}
$$

From Equation $6 \mathcal{L}$ is found to be

$$
\begin{gathered}
\mathcal{L}_{1}\left(k_{x}, k_{y}, M\right)=\frac{\sqrt{2}}{\pi \sqrt{\bar{k}_{x}\left(1+M_{\infty}\right) \Theta_{1}}} E^{*}\left(2 \Theta_{1}\right) \mathrm{e}^{i \Theta_{2}} \\
\mathcal{L}_{2}\left(k_{x}, k_{y}, M\right) \approx \frac{e^{i \Theta_{2}}}{\pi \Theta_{1} \sqrt{2 \pi \bar{k}_{x}\left(1+M_{\infty}\right)}}\left\{i \left(1-\mathrm{e}^{\left.-i 2 \Theta_{1}\right)}\right.\right. \\
\left.+(1-i)\left[E^{*}\left(4 \bar{k}_{x}^{*} M_{\infty}\right)-\frac{\sqrt{2} \mathrm{e}^{-i 2 \Theta_{1}}}{\sqrt{1+\left(M / M_{\infty}\right)(x / \sigma)}} E^{*}\left(2 \bar{\mu}\left(\frac{M_{\infty}}{M}+\frac{x}{\sigma}\right)\right)\right]\right\}
\end{gathered}
$$

where$$
\Theta_{1} \equiv \bar{\mu}\left(\frac{M_{\infty}}{M}-\frac{X}{\sigma}\right)
$$$$
\Theta_{2} \equiv \bar{k}_{x}+\bar{\mu}(M-x / \sigma)-\pi / 4
$$ 
The above equations for $g$ and $\mathcal{L}$ are for an airfoil situated between $-b<x<b$ whereas the computer program calculates values for $\mathcal{L}$. for an airfoil situated between $0<x<c$.

\section{The Velocity Upwash}

The velocity field produced by an ideal vortex in an incompressible fluid is

$$
v_{\theta}=\frac{\Gamma}{2 \pi r}
$$

where $\Gamma$ is the circulation and $\Gamma / 2 \pi$ is the vortex strength. A more realistic model which includes the effects of vorticity and avoids the singularity at $r=0$ (by introducing a decaying radial function to simulate the viscous core) is

$$
v_{\theta}=\left(1+\frac{1}{2 \alpha}\right) \frac{r_{0}}{r} v_{0}\left[1-e^{-\alpha\left(r / r_{0}\right)^{2}}\right]
$$

where $\alpha=1.25643$ is determined by requiring $d v_{\theta} / d r=0$ at $r=r_{0}$. Also, $v_{\theta}=v_{0}$ for $r=r_{0}$ and the circulation $\Gamma_{\infty}$ for large $r$ is $\Gamma_{\infty}=2 \pi(1+0.5 / \alpha) r_{0} v_{0}$. This vortex model has also been used by other authors (refs. 16, 17).

For a flat plate airfoil, only the gust velocity normal to the airfoil surface produces a lift response. If the airfoil were to intersect the vortex normal to the vortex line, no lift would be produced. Thus, it is necessary for the vortex to be tilted out of the normal. The vortex will be assumed to lie in the $x, z$ plane. This will later be extended to permit the vortex to be rotated by the polar angle $\phi_{\mathrm{v}}$ about the $\mathrm{z}$ axis as well, with the airfoil span along the $\mathrm{y}$ axis.

To find the $z$ velocity component for a vortex tilted by $\theta_{v}$ from the $z$ axis and $\phi_{\mathrm{v}}=0$, the radius $\mathrm{r}$ in Equation 22 must be interpreted as the normal distance $R$ to the vortex; i.e.,

$$
R^{2}=\left(x \cos \theta_{v}-z \sin \theta_{v}\right)^{2}+y^{2}
$$

The velocity is then

$$
w=\frac{y}{R} \sin \theta_{v} v_{\theta}(R)
$$


For a potential vortex Equation 21 together with Equation 24 give

$$
w_{p o t}=\frac{y \Gamma}{2 \pi R^{2}} \sin \theta_{v}
$$

This can be readily checked by noting that the velocity potential $\Phi$ for a potential vortex is

$$
\Phi=\frac{\Gamma}{2 \pi} \phi_{0}
$$

where $\phi$ represents the azimuthal angle around the $z^{\prime}$ axis in a coordinate system with $z$ along the vortex. The relation between the primed and unprimed coordinates is

Then, since $\underline{v}=\nabla \Phi$,

$$
\begin{aligned}
& x^{\prime}=x \cos \theta_{v}-z \sin \theta_{v} \\
& y^{\prime}=y \\
& z^{\prime}=x \sin \theta_{v}+z \cos \theta_{v} \\
& \tan \phi_{0}=y^{\prime} / x^{\prime}
\end{aligned}
$$

$$
w=\frac{\partial \Phi}{\partial z}=\frac{\Gamma}{2 \pi}\left[\frac{\partial \phi_{0}}{\partial z^{\prime}} \frac{\partial z^{\prime}}{\partial z}+\frac{\partial \phi_{0}}{\partial x^{\prime}} \frac{\partial x^{\prime}}{\partial z}\right]=\frac{\Gamma}{2 \pi} \sin \theta_{v} \frac{y^{\prime}}{x^{\prime 2}+y^{\prime 2}}
$$

agreeing with Equation 25

In order to represent the upwash field as sinusoidal gusts, the $x, y$ Fourier transform of Equation 24 will be performed. Thus,

$$
\widetilde{w}\left(k_{x}, k_{y}\right)=\frac{\sin \theta_{v}}{(2 \pi)^{2}} \int_{-\infty}^{\infty} \frac{y}{R} v_{\theta}(R) e^{-i\left(k_{x} x+k_{y} y\right)} d x d y
$$

Transforming from an $x, y$ to an $x^{\prime}, y^{\prime}$ integration (noting that the integration is performed in the $z=0$ plane) then to an $R$, $\phi$ integration gives

$$
\tilde{w}\left(k_{x}, k_{y}\right)=\frac{\tan \theta_{v}}{(2 \pi)^{2}} \int_{0}^{\infty} R v_{\theta}(R) d R \int_{0}^{2 \pi} \sin \phi e^{-i\left(k_{x} \frac{\cos \phi}{\cos \theta_{v}}+k_{y} \sin \phi\right) R} d \phi
$$

The $\phi$ integral can be performed for arbitrary $v_{\theta}(R)$. Denoting this integral by $I$,

$$
\begin{aligned}
I & =2 i \int_{0}^{\pi} \sin \phi \sin \left[R\left(k_{x} \cos \phi / \cos \theta_{v}+k_{y} \sin \phi\right)\right] d \phi \\
& =4 i \int_{0}^{1} \cos \left(\xi k_{x} R / \cos \theta_{v}\right) \sin \left(R k_{y} \sqrt{1-\xi^{2}}\right) d \xi
\end{aligned}
$$


This integral is shown on page 399 of reference 20 to be

$$
I=\frac{2 \pi i k_{y}}{\sqrt{k_{y}^{2}+k_{x}^{2} / \cos ^{2} \theta_{v}}} J_{1}\left(R \sqrt{k_{y}^{2}+k_{x}^{2} / \cos ^{2} \theta_{v}}\right)
$$

Substitution of this result into Equation 30 and using Equation 22 for $v_{\theta}(R)$ gives

$$
\begin{aligned}
& \tilde{w}\left(k_{x}, k_{y}\right)=\frac{i k_{y} \sin \theta_{v}}{2 \pi \sqrt{k_{x}^{2}+k_{y}^{2} \cos ^{2} \theta_{v}}}\left(1+\frac{1}{2 a}\right) r_{0} v_{0} \\
& \int_{0}^{\infty}\left[1-e^{-\alpha\left(R / r_{0}\right)^{2}}\right] J_{1}\left(R \sqrt{k_{y}^{2}+k_{x}^{2} / \cos ^{2} \theta_{v}}\right) d R
\end{aligned}
$$

Reference 20 on pages 717 and 940 gives the results

$$
\begin{gathered}
\int_{0}^{\infty} J_{1}(\beta R) e^{-a R^{2}} d R=\frac{1}{\beta} \gamma\left(1, \frac{\beta^{2}}{4 \alpha}\right) \\
\gamma\left(1, \frac{\beta^{2}}{4 a}\right)=1-e^{-\beta^{2} / 4 a} \\
\gamma(1, \infty)=1
\end{gathered}
$$

Equation 33 then becomes

$$
\widetilde{w}\left(k_{x}, k_{y}\right)=\frac{i k_{y}\left(1+\frac{1}{2 a}\right) \tan \theta_{v}}{2 \pi\left(k_{y}{ }^{2}+k_{x}{ }^{2} / \cos ^{2} \theta_{v}\right)} r_{0} v_{0} e^{-\left(k_{y}{ }^{2}+k_{x}{ }^{2} / \cos ^{2} \theta_{v}\right) r_{0}{ }^{2} / 4 a}
$$

This can now be introduced into Equation 8 which, together with the expressions for $\mathcal{L}$ given previously, determines $P(t)$. Note that $\underset{w}{W}$ varies linearly with changes in vortex strength $\left(\Gamma / 2 \pi \propto \mathrm{r}_{\mathrm{o}} \mathrm{v}_{\mathrm{o}}\right)$.

The Velocity Upwash for an Arbitrary Vortex Orientation

The velocity upwash given by Equation 36 is for a line vortex in the $x, z$ plane while the airfoil is along the $y$ axis. It is straightforward to generalize this to the case of an arbitrary orientation of the vortex relative to the airfoil.

Let $\xi, \eta, \zeta$ represent a coordinate system rotated about the $z$ axis by $\phi_{V}$. Then coordinates $x, y, z$ are related to $\xi, \eta, \zeta$ by

$$
\begin{aligned}
& x=\xi \cos \phi_{v}-\eta \sin \phi_{v} \\
& y=\xi \sin \phi_{v}+\eta \cos \phi_{v} \\
& z=\zeta
\end{aligned}
$$


The relation between the wavevectors $k_{x}, k_{y}$ and $k_{\xi}, k_{n}$ for the two coordinate systems are easily related using the basic definition

$$
\begin{gathered}
\overline{\bar{w}}\left(k_{\xi}, k_{\eta}\right)=\left(\frac{1}{2 \pi}\right)^{2} \int_{-\infty}^{\infty} w(\xi, \eta) e^{-i\left(k \xi \xi+k_{\eta} \eta\right)} d \xi d \eta \\
\widetilde{\widetilde{w}}\left(k_{x}, k_{y}\right)=\left(\frac{1}{2 \pi}\right)^{2} \int_{-\infty}^{\infty} w(\xi, \eta) e^{-i\left(k_{x} x+k_{y} y\right)} d x d y \\
=\left(\frac{1}{2 \pi}\right)^{2} \int_{-\infty}^{\infty} w(\xi, \eta) e^{-i \xi\left(k_{x} \cos \phi_{v}+k_{y} \sin \phi_{v}\right)+i \eta\left(k_{x} \sin \phi_{v}-k_{y} \cos \theta_{v}\right)} d \xi d \eta
\end{gathered}
$$

where the double bar over $w$ denotes a Fourier transform in $\zeta$ and $n$ and the double wavy bar indicates a Fourier transform in $\mathrm{x}$ and $\mathrm{y}$.

Comparing these two equations shows that

$$
\overline{\bar{w}}\left(k_{\xi}, k_{\eta}\right)=\widetilde{w}\left(k_{x}, k_{y}\right)
$$

if

$$
\begin{aligned}
& k_{\xi}=k_{x} \cos \phi_{v}+k_{y} \sin \phi_{v} \\
& k_{\eta}=-k_{x} \sin \phi_{v}+k_{y} \cos \phi_{v}
\end{aligned}
$$

If $k_{x}$ and $k_{y}$ in Equation 36 are replaced by $k_{\xi}$ and $k_{n}$, then Equation 40 will give $\tilde{w}\left(k_{x}, k_{y}\right)$ for an arbitrary vortex orientation.

\section{Application of the Theory}

Acoustic Signature Calculation - Prediction of the time dependent far field acoustic pressure signature generated by the blade vortex interaction mechanism was based on Equation 8. The integral in this equation was evaluated using a Fast Fourier Transform. The effective lift function, $\mathcal{L}$, in this expression was determined from either Equations 15 or 19 depending on the wavenumber, $k_{x}$. Wavenumber spectrum, $\tilde{w}$, was based on Equation 36 with $k_{x}$ and $k_{y}$ replaced by $k_{\xi}$ and $k_{\eta}$ (eq. 40) in the case of an arbitrary orientation between the vortex and the airfoil.

Acoustic Spectrum Calculation - The harmonic level of the tones generated by multiple chopping of the vortex was predicted using Equation 8 . This was accomplished by recognizing that by dropping the integral over $k_{x}\left(k_{x}=w / u\right)$ there remains a function $\widetilde{\mathrm{P}}_{1}(\omega)$ which is related to the sound spectrum of a single vortex intersection. Since $\widetilde{\mathrm{P}}_{1}(\omega)$ rep-esents a single blade vortex interaction, this function is continuous in the frequency domain rather than 
representing a discrete function with non-zero values only at the blade passage frequency and its harmonics. The continuity of $\widetilde{\mathrm{P}}_{1}(\omega)$ allows extending the analysis to the case of multiple chopping of the vortex. This is accomplished by assuming that the vortex intersections are independent events. Then, if $R_{1}(\tau)$ represents the autocorrelation of the far field sound of a single vortex interaction and $R_{\infty}(\tau)$ the autocorrelation of an infinite series of intersections, $R_{\infty}$ can be defined by an infinite series of the functions $R_{1}$ separated by the time of blade passage; i.e.,

$$
R_{\infty}(\tau)=\sum_{n=-\infty}^{\infty} R_{1}(\tau-2 \pi n / B \Omega)
$$

where $B$ is the blade number and $\Omega$ the rotational frequency in radians/sec. Taking the Fourier transform gives sound spectrum, $S_{\infty}$, for multiple intersection

$$
S_{\infty}(\omega)=B \Omega \sum_{n=-\infty}^{\infty} S_{1}(n B \Omega) \delta(\omega-n B \Omega)
$$

Here $S_{1}(\omega)$ is the spectrum for a single vortex interaction and can be related to the known function $\widetilde{\mathrm{P}}_{1}(\omega)$ by expressing the autocorrelation as

$$
R_{1}(\tau)=\lim _{T \rightarrow \infty}\left[\frac{1}{2 T} \int_{-T}^{T} P_{1}(t) P_{1}(t-\tau) d t\right]
$$

Taking the Fourier transform gives

$$
S_{1}(\omega)=B \Omega \widetilde{P}_{1}(\omega) \widetilde{P}_{1}^{*}(\omega)
$$

Then, combining Equations (42) and (44), transforming to a single sided spectrum function, $G$, and expressing the results in terms of unit frequency (rather than unit radian frequency) gives

$$
G_{\infty}(f)=2 B^{2} \Omega^{2} \sum_{n=-\infty}^{\infty} \widetilde{P}_{1}(n B \Omega) \tilde{P}_{1}^{*}(n B \Omega) 8\left(f-\frac{n B \Omega}{2 \pi}\right)
$$

This represents the final equation used to calculate the acoustic spectrum generated by multiple blade vortex intersections. Note that the delta function is $\delta(f)$ in Eq. (45) rather than $\delta(\omega)$ in Eq. (42) and recall that $\delta(\omega)=$ $\delta(2 \pi f)=\delta(f) / 2 \pi$. 


\section{DESCRIPTION OF THE EXPERIMENT}

\section{Acoustic Research Tunnel}

Operating Characteristics - The experimental study was conducted in the UTRC Acoustic Research Tunnel. A detailed description of the facility is given in reference 24. The tunnel, shown schematically in Figure 5, is of an open-circuit, open-jet design. The inlet is provided with a high length-to-diameter ratio honeycomb section and a series of turbulence suppression screens. These features, in conjunction with a large tunnel contraction, provide a spatially uniform, temporally steady flow with a controlled test section turbulence level of approximately 0.2 percent. Turbulence generators and grids can be inserted upstream of the nozzle to generate wake profiles and a range of turbulence levels in the test section.

The open jet test section is surrounded by a sealed anechoic chamber $4.9 \mathrm{~m}$ high, $5.5 \mathrm{~m}$ long (jet centerline direction), and $6.7 \mathrm{~m}$ wide. The chamber walls are lined with $0.5 \mathrm{~m}$ high fiberglass wedges which provide an anechoic acoustic environment above $175 \mathrm{~Hz}$. Downstream of the test section the airflow enters a diffuser by way of a circular collector that has anechoic treatment on its flow impingement surface. The diffuser operates unstalled and thus is not a major source of background noise. To avoid tunnel $\mathrm{fan}$ noise from propagating upstream into the anechoic chamber, a Z-shaped muffling section with two right-angle bends and parallel treated baffles is located between the diffuser and the fan. The $1100 \mathrm{~kW}$ centrifugal fan exhausts to the atmosphere through an exhaust tower.

Tunnel speed is determined from total pressure measurements upstream of the contracting inlet and static pressure measurements within the sealed anechoic chamber. Since losses are confined to the boundary layer, total pressure upstream and downstream of the contraction are predicted and have been verified, to be equal. The test section velocity has been shown to be temporally steady.

Open Jet Geometry and Test Section Arrangement - A circular inlet nozzle with a $1.28 \mathrm{~m}$ diameter was used in the test program. Initial facility tests described in reference 24 identified an acoustic coupling between the inlet nozzle and the downstream collector lip resulting in edge tones at high tunnel speeds. Triangular tabs were used previously to suppress the acoustic feedback mechanism. However, several facility changes performed in 1979 eliminated the acoustic coupling problem permitting the nozzle to be operated without tabs.

Figure 7 shows the open jet test section arrangement. A NACA 0012 model airfoil was located at the exit plane of the open jet nozzle. The $11.4 \mathrm{~cm}$ chord airfoil generated a tip vortex which was convected into the downstream rotor. The model airfoil was mounted in a clamping device which permitted changing the angle of attack, $\alpha_{2}$, and the vertical flow field penetration height, $h$. Variations in $h$ 
controlled the vortex radial intersection station on the rotor blade, (I), and the intersection angle, $B_{1}$. The plan view of Figure 7 shows a second vortex intersection angle, $\gamma$, which was also monitored.

It was recognized during initial planning stages that changes in $h$ and $\alpha_{2}$ altered the tip vortex strength. The complicated coupling effects between $h$ and $\alpha_{2}$ in addition to the effect of changing rotor blade tip Mach number $\left(M_{\mathrm{T}}\right)$ and blade pitch angle, $\alpha_{1}$, are discussed in the subsection titled Test Program.

The airfoil and rotor shown in Figure 7 were stationed on the open jet centerline with the rotor hub located $0.58 \mathrm{~m}$ downstream of the airfoil trailing edge. This station was selected based on the previous study of Paterson and Amiet (ref. 3) which employed the same rotor model. Preliminary measurements conducted during their investigation showed that the rotor ingested the open jet shear layer when stationed at approximately $0.9 \mathrm{~m}$ downstream of the open jet exit plane. Significant turbulence ingestion noise was generated under this condition. This problem was eliminated by moving the rotor upstream to $0.58 \mathrm{~m}$ (ref. 3). The absence of turbulence ingestion, noise at this station was verified experimentally for the present tip Mach number operating range, which was on1y 10 percent higher than reference 3 .

\section{Model Rotor}

The model helicopter rotor tested in the present study employed a $0.76 \mathrm{~m}$ diameter rotor. The articulated hub was provided with NACA 0012 untwisted blades of $5.1 \mathrm{~cm}$ chord. Blade pitch angle was set with a blade profile template and an inclinometer. Blade-to-blade variations in pitch angle were limited to \pm 10 minutes. This was sufficient to avoid extraneous tones at multiples of shaft frequency.

Rotor tip speed (or revolutions per second, RPS) was set using an adjustable speed control with a $1 \mathrm{~Hz}$ resolution. A once per revolution short duration pulse generated by an optical sensor was used to monitor the speed. This time marker signal was also recorded simultaneously, on analog tape, with the acoustic signal from each microphone. The marker signal was later used as a trigger to initiate blade vortex interaction acoustic signature ensemble averaging.

The small rotor blade chord tested here resulted in Reynolds numbers, which varied, with rotor RPM, from $4 \times 10^{5}$ to $6.5 \times 10^{5}$ at the tip and $9.5 \times 10^{4}$ to $1.5 \times 10^{5}$ at the blade root. Over this Reynolds number range boundary layers on the blade remained laminar for the blade pitch angles investigated. Laminar flow over the blade trailing edge generated vortex shedding noise which was observed as a broad peak in preliminary acoustic spectra. The apparent broadband nature of this noise mechanism was explained earlier by Paterson and Amiet (ref. 3), to be caused by the Doppler shift of discrete tones generated by blades 
approaching and retreating from a fixed microphone. In addition, the spanwise varying velocity on the rotor blade generated a range of discrete tones which further broadened the spectrum.

From a practical standpoint the vortex shedding mechanism has not been identified as an important noise mechanism on full scale helicopters. Furthermore, preliminary acoustic measurements obtained during the present model study indicated that the broad peak masked the tones generated by the blade-vortex interaction phenomenon. It was, therefore, necessary to trip the boundary layers on the model rotor blades and force transition to turbulence to eliminate the laminar boundary layer vortex shedding noise mechanism.

The boundary layer trip procedure employed here was identical to the previously reported approach of Paterson and Amlet. The method represents a variant of the technique first reported by Hama in reference 25 . In the present study a strip of adhesive aluminum tape was cut with pinking shears and installed on the pressure and suction surfaces of each blade at the 25 percent chord station (Fig. 3). Vortex shedding noise was eliminated as verified in the subsection titled Test Program. The present boundary layer trip approach has the advantage of being repeatable. It also avoids the question of uniformity on each blade and similarity between blades when using the distributed roughness approach.

\section{Instrumentation}

Acoustic Measurements - Far-field rotor noise was measured with $0.635 \mathrm{~cm}$ diameter microphones at grazing incidence. Frequency response on each microphone was flat over the $175 \mathrm{~Hz}$ to $10 \mathrm{kHz}$ range of the present study. Microphone signals were amplified and then recorded on magnetic tape. Dynamic response for the FM tape recorder system was flat over the acoustic frequency range investigated. Corrections for atmospheric attenuation were not applied due to the small acoustic source-to-microphone propagation distance and the low acoustic source frequencies.

Source directivity information was obtained by locating microphones on a $3 \mathrm{~m}$ or $2.5 \mathrm{~m}$ arc (Fig. 8a). The microphones formed a horizontal plane which passed through the rotor rig centerline. Measurement angles, $\theta_{0}$, given in Figure $8 a$ are referenced to the upstream rotor axis. Angles ranging from $\theta_{0}=60^{\circ}$ to $140^{\circ}$ provided data from $30^{\circ}$ forward of the rotor plane of rotation to $50^{\circ}$ aft.

A microphone was also located in a vertical plane to provide additional diroctivity information. The unit was placed at $\theta_{0}=130^{\circ}$ and $\phi_{0}=135 \mathrm{deg}$ where $\phi_{0}$ defines an angle measured relative to the horizontal plane through the rotor rig centerline (Fig. 8b).

It should be noted that the horizontal reference plane selected for $\phi_{0}$ here is different from the reference plane used in the blade vortex interaction theory. In the latter case, the reference plane passes through the blade vortex 
intersection station which is also indicated in Figure $8 \mathrm{~b}$. Since the vortex intersection occurred at approximately $0.32 \mathrm{~m}$ below the rotor centerline the difference between the two values of $\phi_{0}$ is less than $6^{\circ}$ for microphones on a $3 \mathrm{~m}$ arc. Thus, the definition of the microphone orientation angle, $\phi_{0}$, can be considered to be similar for the present theory and experiment.

It is important to recognize that the microphone measurement angle, $\theta_{0}$, in the experimental study coincides with the observer angle, $\theta_{0}$, in the theoretical formulation (Fig. 4) only if certain conditions are satisfied for blade pitch angle, $\alpha_{1}$, and the azimuthal microphone angle, $\phi_{0}$. The conditions are $\alpha_{1}=0^{\circ}$ and $\phi_{0}=0^{\circ}$ or $180^{\circ}$. The latter requirement for $\phi_{0}$ was satisfied for the microphones located in the horizontal plane as indicated by the arguments in the above paragraph. But, $\alpha_{1}$ varied from $5^{\circ}$ to $14^{\circ}$. Values of $\theta_{0}$ associated with the experiment and theory, consequently, differed by a value of $\alpha_{1}$. Input values of $\theta_{0}$ for the present theoretical prediction must, therefore, be increased by $\alpha_{1}$ to account for this difference. In the general case where the above condition on $\phi_{O}$ is not satisfied, microphone measurement angles must be defined directly in terms of the blade referenced observer angles in Figure 4. Otherwise, an analytical transformation is needed to convert measurement angles to blade referenced angles.

Spectrum analysis was conducted with an 800 line Fast Fourier Transform spectrum analyzer ( 400 line in dual channel mode). The effective bandwidth was 12.5 $\mathrm{Hz}$ for the 0-10 kHz analysis range. Bandwidths for other frequency ranges given in this report are determined by direct proportion. A high resolution capability in the frequency domain of the spectrum analyzer was also used to evaluate narrowband random features of rotor tone noise at high blade passing harmonics. The analyzer bandwidth for this data is given in the corresponding figure.

Vortex Velocity Field Measurements - Mean velocity measurements of the vortex velcolty field were obtained using a single0.025 mm diameter (with a $0.5 \mathrm{~mm}$ sensing length) linearized hot film probe with the sensor slated at $45^{\circ}$ to the open jet centerline. The single element sensor was used to decompose the total vortex velocity vector into the azimuthal $\left(V_{\theta}\right)$ and axial (U) components shown in Figure 9. The probe was oriented with the $0.5 \mathrm{~mm}$ sensing length parallel to the local $v_{\theta}$ vector. Details of the voretx measurement procedure are given in Appendix A. It should be noted that a crossed probe could not be used for surveying the vortex since the large spacing between the two sensor elements $(1 \mathrm{~mm})$ would average $\mathrm{v}_{\theta}$ information inside the small radius $(0.4 \mathrm{~cm})$ viscous core.

Dependence of the vortex velocity field on radial distance from the vortex centerline was obtained by traversing the hot film probe through the vortex on a line perpendicular to the vortex center. These traverses were conducted using a two-directional linear traverse system $( \pm 0.0013 \mathrm{~cm}$ position accuracy) coupled with 
two variable speed stepping motors and a two digital encorders $(0.0013 \mathrm{~cm}$ resolution). The traverse system, hot film probe and upsteram semispan airfoil are shown in Figure 10.

Before conducting the above-described radial traverse through the vortex it was necessary to locate the vortex center. Although the approximate location of the tip vortex from the upstream airfoil was known, its precise position in space could not be determined in advance. Alignment of the hot film probe with the upstream airfoil tip was not an acceptable solution to this problem. This is because of the small size of the viscous core (approximately $0.4 \mathrm{~cm}$ radius) and the airfoil downwash velocity. The latter phenomenon translated the vortex center as the flow was convected from the blade trailing edge to the rotor plane. A procedure was developed, therefore, to locate the vortex center at the rotor plane (Appendix A).

Turbulence intensity and length scale in the vortex viscous core were estimated from hot film crossed probe measurements. Limitations of the accuracy of this data due to sensor spacing must be recognized when interpreting this data. Linearizers and a sum and difference circuit were used in conjunction with a true root-meansquare meter to estimate the azimuthal and axial turbulence intensity in the viscous core. Similar to the mean velocity measurements, the crossed probe was traversed on a straight line through the vortex center to obtain the radial variations of the turbulence field. Turbulence integral length scale on the vortex centerline was determined using a 400 line real time correlator and probability analyzer.

Flow Visualization - The objective of the flow visualization phase of the study was to document the details of the blade vortex intersection. The vortex pathline at the rotor plane was determined by injecting smoke into the tip vortex at its inception point on the upstream semispan airfoil. Smoke was released from a small elongated slit on the rounded tip of the airfoil. Smoke ejection rate was adjusted to avoid generating a high speed jet which would alter the vortex formation. A closeup of the smoke ejection is shown in the photographs of Figure 11.

The smoke pathline was documented with a camera mounted in the rotor plane of rotation. A short duration light pulse was used to illuminate the smoke. For each test condition photographs were obtained in the vertical plane to document the vortex intersection angle, $\beta_{1}$, and the intersection station, $I$, shown in Figure $7 \mathrm{~h}$. In addition, photographs in a horizontal plane recorded the intersection angle, $\gamma$, shown in Figure $7 \mathrm{a}$. This latter angle required documentation because the strength of the semi-span airfoil downwash at the rotor plane was unknown. A general conclusion, however, was that $\gamma=0^{\circ}$ for all rotor operating conditions. All values of $\beta_{1}$ and $\gamma$ are based on averaging three photographs of each test condition. Resolution was estimated at $\pm 0.5^{\circ}$. 
A sample photograph obtained in the vertical plane is shown in Figure 12. The upstream airfoil appears on the left with tufts attached to the trailing edge to verify that the flow remained attached and the tip vortex was steady. Vortex unsteadiness was a concern for the small airfoil aspect ratios (small values of penetration height, $h$ ) used to demonstrate the absence of blade vortex interaction noise when the vortex passed outside the rotor disk.

Blade Tracking and Coning - Rotor blade tracking was evaluated during preliminary tests using a video camera and video monitoring screen. Synchronized light pulses simultaneously superimposed all four blades on the monitor screen. The camera viewing angle and synchronization provided a cross section view of the squared off blade tip. The sharp trailing edge on the monitor image indicated visually that all rotor blades were rotating in the same plane and blade pitch angles were identical. The accuracy of this visual assessment was based on observing a trailing edge thickness (of the superimposed blades) of less than 1 percent of the blade chord. This corresponds to a 16 minute variation between blade pitch angles which is within the \pm 10 minute limit used in setting pitch angle. The monitor image of the blade chord was $10 \mathrm{~cm}$ during this evaluation.

Blade coning angles were also determined with the video system. Maximum coning angles of $0.5^{\circ}$ indicated that the blades essentially rotated in a plane perpendicular to the rotor drive shaft. Although blade coning effects were small, they were accounted for when determining the vortex intersection angle, $\beta_{1}$, from flow visualization photographs. The procedure involved referencing $\beta_{1}$ to the local blade section in each photograph.

\section{Test Program}

Rational for Test Configuration - Before describing the rational for selecting the present vertical ascent test configuration it is necessary to describe the tail rotor blade vortex interaction noise mechanism. Figure 13a shows a simplified main rotor tip vortex trajectory as it is convected through the tail rotor. Tip vortices which initially are nearly normal to the tail rotor plane of rotation (defined by the coordinates $x_{1}, x_{2}$ for the case of zero cant angle on the rotor) are turned or skewed by the induced flow through the tail rotor. The skewed vortex velocity field subsequently creates an unsteady velocity component normal to the blade planform which results in noise radiation. Without turning of the flow, the vortex centerline remains normal to the plane of rotation and the transverse velcoity components are small. Under this condition tail rotor blade vortex interaction noise is weak. (Note that small transverse velocity components can exist in this case, due to the blade pitch angle or twist and the axial mean velocity defect (if a defect exists) in the vortex core.) 
The experimental method reported here permits varying the angle between the vortex centerline and the normal to the blade planform. The approach employs the simple but tractable test configuration shown in Figures 2 and 3 . Here, skewing of the vortex centerline is achieved by contraction of the flow through the rotor as illustrated in Figure 13b. Vortex skewing relative to the blade normal now occurs in the plane defined by the $x_{2}, x_{3}$ axes. Differences between the directions of the convected mean flow in Figures $13 \mathrm{a}$ and $13 \mathrm{~b}$ only change the blade relative Mach number, and, hence, loading noise. The present test configuration, therefore, permits direct assessment of the noise mechanism.

It should be noted that the model rotor shown in Figure $13 \mathrm{~b}$ could be oriented with the rotor shaft at $90^{\circ}$ to the open jet flow. This geometry would then simulate the tail rotor forward flight operating condition shown in Figure 13a. However, the upstream airfoil vortex trajectory would then be in the plane of the rotor Ins $\mathrm{tead}$ of at $90^{\circ}$ to the rotor as Figure 13a. Thus, the experimental arrangement in Figure 13b represents the closest simulation of the actual tail rotor interaction with main rotor shed vortices.

Coupling Effects Between Aerodynamic Parameters - It was desired to devise an experiment in which only one parameter varied. This was important because the vortex and rotor blade aerodynamic parameters were coupled. The following discussion defines the coupling effects which required consideration when formulating the test matrix.

First, changing the semispan airfoil angle of attack, $\alpha_{2}$, or the airfoil penetration height, $h$, changes the vortex strength. A further change in vortex strength would also appear to occur due to the mean flow contraction induced by the rotor. (This point is discussed in the section titled Velocity Field Incident on Rotor.)

Second, changing blade number, $B$, pitch angle, $\alpha_{1}$, or tip Mach number, ${ }_{\mathrm{T}}$, changes the rotor induced contraction ratio. Consequently, vortex intersection station, $I$, and intersection angle, $B_{1}$, are changed.

Due to the complicated coupling between aerodynamic parameters, experimental assessment of the blade vortex interaction noise mechanism necessitated a specific test program sequence. Parameters which had a minimum coupling effect were assessed first. For example, blade number was tested with a minimum change in vortex strength or intersection angle, $\beta_{1}$. Conclusions obtained from the blade number sensitivity test were then used in the assessment of other parameters.

To illustrate the approach, blade tip Mach number dependence was investigated by changing $M_{T}$ and blade number. The latter change was required to maintain a constant contraction ratio, thereby ensuring fixed values for the intersection station, $I$, and intersection angle, $B_{1}$. When necessary, airfoil penetration height, $h$, was also changed to hold $I$ (or $\beta_{1}$ ) constant. The subsequent change in vortex strength due to varying $h$ was documented with the independent vortex velocity field measurements. 
Formulation of Test Matrix - The range of the parameters investigated experimentally can now be specified and the rationale will be outlined where necessary. Open jet speed, $U_{0}$, was selected to be $9 \mathrm{~m} / \mathrm{sec}$. A low velocity was chosen since it created a large rotor inflow contraction ratio. This ensured that the vortex centerline would be skewed relative to the normal to the rotor blade planform.

The effect of vortex strength was evaluated by setting different angles of attack on the upstream airfoil $\left(\alpha_{2}=0^{\circ}, 6^{\circ}, 12^{\circ}\right)$. Flow visualization using tufts verified that the airfoil operated unstalled at all values of $\alpha_{2}$. Although NACA 0012 airfoils typically operated unstalled for $\alpha_{2}>12^{\circ}$, larger angles of attack were not tested. This decision was based on the tuft motion which indicated the existence of an unsteady tip vortex at the 1 ow $9 \mathrm{~m} / \mathrm{sec}$ open jet speed. Such unsteadiness could result in undesirable wandering of the vortex at the rotor plane. This condition was most noticeable at small airfoil penetration heights, $h$, corresponding to small airfoil aspect ratios.

Blade vortex intersection station, $I$, was selected to be 1.25 rotor blade chords inboard from the blade tip. At this station, three dimensional tip effects not accounted for in the acoustic theory were expected to be weak. Intersections further inboard (larger values of $I / R_{1}$ ) were undesirable since the associated decrease in local Mach number results in weak blade vortex interaction noise relative to blade broadband noise. More important, at larger values of $I / R_{I}$ the rotor blade intersects the upstream airfoil two dimensional wake which introduces an additional noise mechanism. Blade intersection station was held constant by adjustment of blade penetration height, $h$, as described below in the discussions of the remaining parameters.

Blade number dependence, $B$, was evaluated using a two and four bladed rotor configuration. Due to coupling between aerodynamic parameters, changes in $B$ also changed the open jet contraction ratio and, hence, the vortex strength, intersection station, $I$, and intersection angle, $\beta_{1}$. Changes in $I$ and $\beta_{1}$ were countered by a small adjustment of the alrfoll penetration height, $h$. Changes in vortex strength were shown to be negligible.

The influence of vortex intersection angle, $\beta_{1}$, was determined by combined changes in $h, B$, and $\alpha_{1}$. Changes in $h$ (or the separation distance, $H$, in Figure $7 b$ ) caused the vortex to be convected on different path lines into the rotor. For a pathline originating inside the rotor radius ( $H$ positive) the vortex was convected through the rotor with minimum contraction resulting in $\beta_{1}=0^{\circ}$. In contrast, displacing the airfoil to negative values of $\mathrm{H}$ in Figure $7 \mathrm{~b}$ and changing from $B=2$ to $B=4$ created a higher contraction ratio. In this way $B_{1}$ was varied from $0^{\circ}$ to $14.6^{\circ}$. Blade number effects were accounted for using the previous independent assessment of this parameter. Changes in vortex strength were shown to be negligible. 
Blade pitch angle and Mach number investigations also required additional changes in $B$ and $h$ to hold $I$ and $B_{1}$ constant. Pitch angle varied from $5^{\circ}$ to $14^{\circ}$ blade geometric angle of attack. Mach number varied over a range of $\mathrm{M}_{\mathrm{T}}=0.35$ to 0.55 . The lower value was limited by merger of the blade vortex interaction tones and the rotor broadband noise. The upper value was limited by the generation of blade thickness noise at higher Mach numbers. Introduction of a second competing noise mechanism would complicate efforts to isolate the blade vortex interaction noise mechanism.

Forward Flight Effects - For tests conducted at freestream Mach numbers below 0.1 , measurements outside an open jet can be used to infer the acoustic source characteristics. This was verified experimentally by Schlinker and Amiet (ref. 26) using experiments designed to assess the effects of shear layer refraction, reflection, and scattering of sound. Based on their conclusions, microphone corrections were not needed for the present experimental study.

\section{Qualification of Acoustic Test Procedure}

Several preliminary acoustic measurements were conducted to establish the quality and limitations of the acoustic measurements. Facility and rotor rig background noise are documented in Figure 14. Here the rotor rig was operated without a hub and the upstream semispan afrfoil was removed. Rotor speed was varied over the selected test range with tunnel speed set at $9 \mathrm{~m} / \mathrm{sec}$. Achieving the low background noise shown in the present figure required wrapping the rotor rig with commercially available $0.15 \mathrm{~cm}$ lead sheets covered with sound absorbing foam. This covering (not shown in Figs. 2 or 3 ) attenuated noise generated by internal rotating components such as the bearings on the rotor rig. The resulting background noise level was sufficiently below the rotor broadband noise levels as indicated by acoustic measurements presented in later sections.

The accuracy of setting blade pitch angle and the close blade tracking are documented in Figure 15a. Discrete tones shown here represent blade loading noise since the semispan airfoil was removed. Accurate blade-to-blade pitch angle settings and the close blade tracking is verified by the absence of a discrete tone at the rotor shaft frequency. Additional verification is evident in the absence of higher shaft harmonics. Finally, increasing blade number from $B=2$ to $B=4$ (Fig. 15b) provided a $6 \mathrm{~dB}$ increase in loading noise. This increase occurs only if all blades are operating at the same pitch angles. As in Figure 15a, discrete tones at shaft harmonics are absent for $B=4$. It should be noted that the steady loading tones decay rapidly with harmonic number as expected from theory. 
Figure 15a also confirms the absence of higher harmonic narrowband random noise due to ingestion of the open jet turbulent shear layer at $M_{T}=0.39$.

Increasing the blade tip. speed to $\mathrm{M}_{\mathrm{T}}=0.55$, as shown in Figure 16, again verifies the absence of turbulence ingestion noise and demonstrates that close blade tracking was retained at high tip speeds. 
VELOCITY FIELD INCIDENT ON THE ROTOR

\section{Objective}

The objective of the velocity measurements was to document the steady and unsteady flow field incident on the rotor. Steady velocities investigated consisted of the azimuthal velocity $\left(V_{\theta}\right)$ and the axial velocity: defect associated with the vortex. In addition, the two-dimensional semispan airfoil wake axial velocity defect was surveyed. Unsteady velocity measurements included the turbulence intensity and length scales in the viscous core.

Measurements of the azimuthal velocity component were used to assess the vortex velocity field model in the blade vortex interaction theory. In particular, these measurements were needed to verify the radial dependence of the axisymetric flow assumptions in Equation 22. In addition, the velocity measurements determined the maximum azimuthal velocity, $\mathrm{V}_{0}$, at the edge of the vortex viscous core and the viscous core radius, $r_{0}$. These parameters were experimental inputs in the assessment of the acoustic theory.

In essence, the above described measurements of the azimuthal velocity field documented if the vortex was fully-developed. An experimentally generated fullydeveloped vortex was critical since the present noise theory does not treat the intermediate stage between the initially flat trailing vortex sheet and the fully rolled up vortex. Fortunately, experimental evidence indicates that vortex rollup occurs within a few blade chords. Thus, the vortex development was not expected to be a limitation in the present experiment since the rotor was stationed 5 chords downstream of semispan airfoil trailing edge.

Documentation of the axial velocity defects in the vortex and two dimensional wake were required since these velocity fields could potentially introduce additional unsteady upwash components sensed by the rotor. The two dimensional wake was included in this evaluation since the rotor blade vortex intersection occurred at 1.25 chords inboard of the blade tip. Under this condition, it was possible that the rotor blade interacted with the two dimensional wake resulting in a wake chopping mechanism similar to that occurring in turbo-fan engines equipped with inlet guide vanes. Since the present vortex interaction theory models only the blade response to $\mathrm{v}_{\theta}$, it was essential to document the various axial velocity defects.

Measurements of the vortex viscous core turbulence intensity and length scale were needed to assess the importance of incldent turbulence noise. Potentially, blade-to-blade correlations existed due to multiple chopping of turbulent eddies in the viscous core. Under this condition, narrowband random peaks could occur at the same blade passing harmonics generated by chopping the vortex azimuthal velocity fleld. 
Approach

Steady and unsteady velocity measurements were conducted at the rotor plane 5 chords downstream of the semispan airfoil. All surveys were performed in a plane perpendicular to the open jet centerline with the rotor removed.

Several reasons existed for removing the rotor during the velocity surveys. First, the present single element slanted hot film probe measurement technique assumes nonexistent radial velocities in the flow field. This condition was not satisfied with the rotor installed since the flow was contracted to skew the vortex centerline relative to the blade chord normal. A crossed-probe sensor can be used to circumvent this limitation but such a sensor averages radial velocity information (Appendix A) in the vortex core. A second reason for removing the rotor was the danger involved with measuring the vortex velocity field at the rotor face. During operation of the facility any mismatch between the rotor rotation speed and the tunnel speed results in coning of the rotor blades. Potentially, the blades could strike the velocity sensing probe. A final reason for removal of the rotor was to avoid contaminating the unsteady hot film velocity measurements with the particle velocity generated by the rotor acoustic field.

\section{Vortex Azimuthal and Axial Mean Velocity Field}

Radial velocity dependence of the vortex azimuthal velocity component, $v_{\theta}$, is shown in Figure 17. Here, $V_{\theta}$, is normalized by the open jet freestream velocity, $\mathrm{U}_{\mathrm{O}}$, while radial distance from the vortex center (defined schematically in Figure 9) is normalized by the semispan airfoil. Measurements shown here were obtained in the horizontal plane defined by the $x, z$ axes in Flgure 9 . The vortex center is defined as the point at which $V_{\theta}$ is zero. This provides a check on the ability to Independently Identify the vortex center using the approach defined in Appendix A.

The velocity profiles in Figure 17 correspond to two airfoil penetration heights, $h$, or airfoil to rotor tip separation distances, H. A high resolution radial traverse is provided for each value of $\mathrm{H}$ to show the velocity profile inside the vortex viscous core. In each case, airfoil angle of attack is constant and corresponds to $\alpha_{2}=12^{\circ}$.

The profiles in Figure 17 show a systematic change in vortex structure over the range of separation distances tested. Decreasing the penetration height, or the airfoil aspect ratio (AR), decreased the maximum tangential velocity. This is expected since AR controls the strength of the vortex sheet which rolls up into the tip vortex. Viscous core diameter, $D$, normalized by airfoil chord $c_{2}$, is shown to remain approximately constant with $\mathrm{D} / \mathrm{c}_{2}=0.09$. 
Precise symmetry of the velocity field does not exist since the maximum positive and negative values of $v_{\theta} / \mathrm{U}_{0}$ differ. In addition, decay of the potential velocity field outside the viscous core is slightly different on the pressure and suction sides of the airfoil. The degree of asymmetry can be evaluated by comparing the measured velocity field with the analytical velocity field given by Equation 22 . Figures $17 \mathrm{a}$ show the calculated $\mathrm{v}_{\theta}$ velocity profile based on the average value of the maximum positive and negative velocity and the measured core diameter $\left(\mathrm{D} / \mathrm{c}_{2}=\right.$ 0.091). Although complete symmetry does not exist the predicted velocity gradient in the viscous core and the potential flow decay rate were considered to adequately model the vortex.

Further assessment of the azimuthal velocity field is provided by the multiple traverses shown in Figure 18. Here traverses were initiated at different radial stations designated by the numbers 1 through 8 in the small insert showing the airfoil. In each case, the traverse started at a station in the potential velocity field and penetrated the vortex core. The series of curves has been shifted to allow plotting all velocity profiles on the same page. The vortex center is indicated on each trace. All traces have been plotted showing the absolute value of $v_{\theta}$.

Figure 18 indicates that the vortex is fully developed at the rotor plane since the velocity profiles indicate a symmetric flow. The only exceptions to this symmetry are the fine structure of the profiles and the non-zero values of $V_{\theta}$ at large values of $r / c_{2}$ for radial traverses 6,7 and 8 . The non-zero azimuthal velocities are possibly due to the downwash created by the semispan airfoil. This explains the need for flow visualization to establish the vortex intersection angle, $\gamma$, in Figure 7.

Velocity profile surveys such as those in Figures 17 and 18 were used to document the vortex viscous core diameter and the maximum azimuthal velocity. These results are summarized in Figure 19 with data points spanning the range of the airfoil angle of attack and aspect ratios tested in the acoustic study. Lines have been drawn through the data points to permit interpolating the data. This avoided performing the time consuming velocity profile measurements for every test condition in the acoustic study.

The results in Figure 19 indicate that the normalized vortex core diameter varies between $D / c_{2}=0.07$ and 0.09 . Expressed in terms of the rotor blade chord, $c$, these values would be $D / C=0.16$ and 0.20 . Thus, the vortex core is small compared to the rotor blade chord ensuring an impulse response in the acoustic signature. This shifts the tones in the blade vortex interaction spectrum to high frequencies which helps to distinguish this noise from loading noise. In this way, the blade vortex interaction mechanism can be isolated. 
Velocity results in Figure 19 indicate that $v_{\theta} / U_{0}$ is a linear function of airfoil separation heights $H$ or aspect ratio, AR. The only exception to this is the measurement at $\alpha_{2}=6^{\circ}$ and $A R=3.1$. Here $V_{\theta} / U_{0}$ falls below the straight line extrapolation. A similar nonlinear dependence occurs for the vortex core diameter at this test condition. These discrepancies are presently not understood. Blade vortex interaction noise data was not acquired at this test condition.

Axial velocity dependence on distance from the vortex center is shown in Figure 20 for a range of airfoil separation heights, $H$. The profiles indicate a velocity defect in the viscous core with the defect increasing as $\mathrm{H} / \mathrm{c}_{2}$ changes from -0.55 to t0.88. However, the separation heights used in the assessment of the vortex interaction noise mechanism only varied from $\mathrm{H} / \mathrm{c}_{2}=0$ to +0.55 . Over this range the axial velocity defect is approximately 5 percent of the mean velocity level. Note that the value of $\mathrm{H} / \mathrm{C}_{2}$ is given in Table 1 for each test condition. (One test was conducted at $\mathrm{H} / \mathrm{c}_{2}=-0.55$ but this did not generate impulsive noise since the vortex intersected the rotor plane outside the rotor radius.)

Values of $U / U_{0}$ less than unity outside the vortex core in Figure 20 suggest a reduced open jet speed. However, at sufficiently large radial distances, $U / U_{0}$ approached the freestream value. The nonuniform open jet velocity over the distance shown in Figure 20 was not expected to contribute to unsteady blade noise.

Axial Velocity Defect in Two Dimensional Wake

The upstream semispan airfoil wake velocity defect is shown in Figure 21 . Here the normalized parameter, $1-\mathrm{U} / \mathrm{U}_{\mathrm{O}}$, is presented as a function of transverse distance on a line perpendicular to the airfoil planform (based on $\alpha_{2}=0^{\circ}$ ). The measurement was conducted at 0.88 chords inboard of the airfoil tip. The transverse measurement distance is represented as a normalized radial distance to permit direct comparison with the vortex velocity field.

Based on Figure 21, the maximum velocity defect associated with the two dimensional wake corresponds to a 15 percent decrease of the open jet speed. This exceeds the 5 percent axial velocity defect existing in the vortex viscous core. The airfoil wake is, therefore, potentially the strongest source of unsteady axial velocity defects incident on the rotor blade. Note that the location of the airfoil wake velocity defect is shifted relative to the vortex center, possibly due to the airfoil downwash.

For comparison, the radial variation of the vortex azimuthal velocity component is also shown in Figure 21 for the same test condition. Here the magnitude of $\mathrm{V}_{\theta} / \mathrm{U}_{\mathrm{o}}$ exceeds the wake velocity defect suggesting that the rotor blade unsteady upwash will be dominated by the vortex azimuthal velocity field. However, based on a following subsection, titled Unsteady Upwash Due to Blade Vortex Interaction, the fluctuating azimuthal velocity component sensed by the rotor blade is approximately $v_{\theta} \sin \beta_{1}$ where $\beta_{1}$ is the blade vortex intersection angle. As an example, if $\beta_{1}=14.6^{\circ}$ 
(which corresponds to Test Condition B to be discussed later) the normalized unsteady upwash velocity is $\left(V_{\theta} / U_{0}\right) \sin 14.6^{\circ}=0.12$. This value is now comparable to the two dimensional wake axial velocity defect and exemplifies the need to assess the noise generated by the rotor blades intersecting the wake velocity defect.

\section{Turbulence in Viscous Core}

Previous subsections concentrated on the mean velocity field associated with the vortex and the two dimensional wake. It now remains to document the turbulence in the vortex viscous core. Normalized turbulence intensities are presented in Figure 22 for the azimuthal $\left(v_{\theta}^{\prime} / \mathrm{U}_{0}\right)$ and axial $\left(\mathrm{u}^{\prime} / \mathrm{U}_{0}\right)$ velocity components. A comparison of $v_{\theta}^{\prime} / U_{0}$ and $v_{\theta} / U_{0}$ (Fig. 17) indicates that unsteady azimuthal velocities sensed by the rotor are dominated by $v_{\theta}$. A comparison of $u ' / U_{0}$ and the mean axial velocity defect in vortex core and the airfoil wake indicates that unsteadiness due to these velocities is of the same order of magnitude. Thus, the axial turbulence component in the vortex viscous core in addition to the axial mean velocity defects must be considered in the assessment of potential noise sources contaminating the blade vortex interaction noise experiment.

Autocorrelations of the axial component of turbulence were also obtained to determine the eddy length scale associated with the turbulence. Figure 23 shows a sample autocorrelation normalized by the zero delay time value. The Eulerian time scale represented by the area under Figure 23 , is given by $J_{u x}=7 \mathrm{msec}$. Invoking Taylor's frozen flow hypothesis the axial integral length scale becomes $\lambda_{f}=U_{o} J_{u x}=$ $6.3 \mathrm{~cm}$. Based on approximately a $3 \mathrm{msec}$ time between blade vortex intersections (corresponding to Test Condition $B$ in Table 1) the eddy associated with the calculated length scale would be chopped twice. Thus, multiple intersections of the turbulence in the vortex core was considered a potential source of narrowband random noise at blade passing harmonics.

\section{Changes in Velocity Field Due to Contraction of Flow}

Vortex and axial wake velocity measurements were obtained with the rotor removed. The following discussion demonstrates how these measurements are applied in the presence of the mean flow contraction created by the rotor. The discussion begins by considering the azimuthal velocity component, $v_{\theta}$, which forms the basis of the present acoustic theory. The affect of contraction on the axial velocity defect is described 1 ater. 
The contraction skews the initially straight vortex in Figure $13 \mathrm{~b}$ causing the vortex centerline to intersect the rotor at angles $\beta_{1}$ and $\gamma$. The azimuthal velocity field, documented by hot film measurements (without contraction) in the plane normal to the vortex centerline, is similarly reoriented. The effect on the upwash velocity, $\tilde{\tilde{w}}$, at the rotor plane is accounted for theoretically by the intersection angles $\theta_{\mathrm{v}}$ and $\phi_{\mathrm{v}}$ in Equations 36 and 38. Increases in the radial velocity field due to vortex stretching during the contraction are, however, not incorporated in the theory. These changes are known to occur based on Prandtl's model (ref. 27) for a contracting flow. His model predicts that the azimuthal velocity in an axial vortex increases as the square root of the velocity contraction ratio. Since this change could not be monitored experimentally it was necessary to analytically link the velocity fields occurring with the rotor removed and installed.

Before developing the analytical correction to the measured velocity data, it is useful to examine in detail the changes in the unsteady upwash velocity due to changing the azimuthal velocity, $v_{\theta}$, or the vortex core radius, $r_{0}$. Based on Equation 36, changes in $v_{\theta}$ or $r_{0}$ only influence the parameters $r_{0} V_{0}$ and $r_{0}{ }^{2}$. Thus, if the stretched vortex was also defined by the radial velocity function in Equation 22 , predicting the contraction effect would be limited to calculating the changes in $r_{0}$ and $V_{0}$.

It now remains to develop the equations defining the vortex after contraction. Assume initially that the radial velocity function in Equation 22 also models the stretched vortex. (The proposed similarity of the velocity function is a necessary condition for satisfying Kelvin's theorem which is described below.) Then the parameters $r_{0,1}$ and $v_{0,1}$ in Equation 22 are replaced by $r_{2}, r_{0,2}$ and $v_{0,2}$. Here, the subscripts 1 and 2 are used to denote conditions before and after the contraction.

The product, $\mathrm{r}_{0,2} \mathrm{~V}_{0,2}$ can be linked to $\mathrm{r}_{0}, \mathrm{v}_{0,1}$ by using Kelvin's theorem (ref. 28). Based on this theorem, the circulation, $\Gamma$, around any contour line in the fluid remains constant in time as the contour is convected in an inviscid flow. Selecting a contour line at the edge of the viscous core and calculating the circulation before contraction gives $\Gamma_{1}=2 \pi r_{0,1} V_{0,1}$. Similarly, the circulation after contraction is $\Gamma_{2}=2 \pi r_{0,2} V_{0,2}$. The equality of $\Gamma$ required by Kelvin's theorem results in $\mathrm{r}_{0,2} \mathrm{v}_{0,2}=\mathrm{r}_{0,1} \mathrm{v}_{0,1}$. Thus, the product $\mathrm{r}_{0} \mathrm{~V}_{\mathrm{o}}$ in the Equation for $\tilde{\tilde{w}}$ remains invariant as the vortex is contracted.

It should be noted that for an ideal inviscid potential vortex the product, $\mathrm{rV}$, is invariant with radius and represents the vortex strength $\mathrm{K}_{1}=\Gamma / 2 \pi$. For this reason the product $r_{0} V_{0}$ is referred to as the vortex strength in the present study. Numerical values for the product are, however, expressed as a normalized vortex strength $\mathrm{K}_{2}=\mathrm{K}_{1} / \mathrm{c}_{2} \mathrm{U}_{0}$. 
The parameter $r_{0}{ }^{2}$ in Equation 36 must also be linked with conditions prior to contraction. In this case, the incompressible continuity of flow condition can be applied. The radius of the vortex is then expected to decrease with the open jet contraction ratio given by $r_{A} / r_{B}$. Here, $r_{B}$ and $r_{A}$ represent conditions before and after contraction and the ratio is estimated using $r_{A} / r_{B}=\left(R_{1}-I\right) /\left(R_{1}-H\right)$. The vortex core radius $r_{0}$, therefore, varies as $r_{0,2}=r_{0,1}\left(r_{A} / r_{B}\right)$.

The effect of contraction on the axial velocity defects in the vortex core and the two dimensional wake can also be predicted. Based on simplified continuity arguments the axial velocities increase in proportion to $\left(r_{B} / r_{A}\right)^{2}$.

In summary, the above discussions demonstrate that measurements of the vortex velocity field obtained with the rotor removed can be applied for the present contracted flow. Hot film measurements of the product $r_{0} v_{0}$ can be used directly as input to the non-contracted upwash velocity expression given by Equation 36 . The effect of changing $r_{0}$ due to contraction can be accounted for analytically before introducing the experimental value in Equation 36.

\section{Unsteady Upwash Due to Blade Vortex Interaction}

Before describing the experimental acoustic results of this study, it is useful to understand the unsteady upwash sensed by the rotor blade in the present vertical ascent test configuration. Figure 24 shows a plan view, front view and side view of a rotor blade during vortex interaction. (This figure is a detailed representation of the blade vortex interaction for blade number 1 in Figure 7.) The vortex is represented by the azimuthal velocity field, $V$, shown in the plan view. The front view shows four vectors $v_{1}, v_{2}, v_{3}$ and $v_{4}$ which represent $v$ at different azimuthal angles. These vectors lie in a plane perpendicular to the local vortex centerline and the radial distance from the vortex centerline is the same for each vector. Thus, the vectors are of equal magnitude. Note that the local blade angle of attack is zero to simplify this description, although the projected length of the vectors may not be equal.

The side view of Figure 24 can be used to examine the unsteady upwash velocity components sensed by the rotor blade. Here the vortex, represented by the circulation vector, $\Gamma$, intersects the rotor blade at angle $\beta_{1}$. In the plan view the vortex arrives at $\gamma=0^{\circ}$ which corresponds to the present test conditions.

For the zero angle of attack conditions shown here vectors $v_{2}$ and $v_{4}$ generate velocity components parallel to the blade chord. Vectors $v_{1}$ and $v_{3}$, however, generate unsteady upwash components. For example, the side view in Figure 24 shows that the contribution due to $v_{1}$ is $V_{N}=v_{1} \sin \beta_{1}$. Based on this simplified figure, the unsteady upwash is a function of $\sin \beta_{1}$. Each chordwise station experiences a simultaneous upwash and downwash due to vectors $v_{1}$ and $v_{3}$. Also, when the blade 
is at angle of attack, vectors $v_{1}$ and $v_{4}$ contribute to the unsteady velocity field. These three dimensional effects illustrate the complicated unsteady flow associated with the blade vortex interaction mechanism and exemplify the need for the present theoretical formulation. 
EXPERIMENTAL ASSESSMENT OF BLADE

VORTEX INTERACTION NOISE

Objective

The objective of this phase of the study was to experimentally assess the noise generated by the blade-vortex interaction mechanism. Incident vortex characteristics and rotor operating conditions were varied to determine the sensitivity of the noise mechanism to isolated changes of each parameter. Parameters investigated included vortex strength, vortex intersection angle, blade number, blade pitch, tip Mach number, and directivity. Experimental assessment of each physical parameter involved conducting tests at two different operating conditions. Acoustic spectra or acoustic pressure signatures were compared to determine the noise radiation sensitivity to the individual parameters. These experimental results also provided a direct assessment of the theoretical prediction procedure.

\section{Effect of Blade-Vortex Interaction}

Objective and Approach - The objective of this subsection is to describe the general features of blade vortex interaction noise. Acoustic spectra and pressure signatures are presented with the upstream vortex generator removed and installed. Measurements are also presented documenting the noise generated by the rotor blade intersecting the vortex axial velocity defect and the two dimensional wake defect. In addition, noise generation due to blade interaction with turbulence in the vortex core and two dimensional wake is assessed. Finally, the issues of spectrum analyzer filter bandwidth and the potential effect of small changes in rotor speed are addressed.

Experimental Results - Figure 25 compares the acoustic spectra obtained with the upstream vortex generator removed and installed. For the installed case, the airfoil angle of attack was $\alpha_{2}=12^{\circ}$. The four bladed rotor tip Mach number was $\mathrm{M}_{\mathrm{T}}=0.55$ while the blade pitch angle was $\alpha_{1}=9.5^{\circ}$. (Additional parameters are listed in Table 1.) The microphone measurement angle in Figure 25 corresponds to a radiation angle, $\theta_{0}=120^{\circ}$, in the downstream quadrant. This measurement angle was selected instead of the $\theta_{0}=90^{\circ}$ station since the blade-vortex interaction dipcle radiation pattern has a minimum in the plane of rotation.

Figure 25a shows that without the semispan airfoil the acoustic spectrum is dominated by the blade passing frequency (BPF) with higher harmonics decaying rapidly until they merge with the broadband background noise. Since the isolated rotor was operated at sufficiently low tip speeds to avoid generating high speed impulsive noise, the measured spectrum is considered to be dominated by loading noise. 
With the semispan airfoil installed (Fig. 25b) the acoustic spectrum is significantly altered with the generation of numerous high frequency tones at multiples of blade passing frequency. Blade vortex interaction tones are $20 \mathrm{~dB}$ above the isolated rotor broadband noise level. Tones occur over a frequency range which permits distinguishing the interaction mechanism from the loading noise.

A further understanding of this noise mechanism can be obtained by considering the acoustic pressure signatures. Figure 26 shows ensemble averaged pressure waveforms with the semispan airfoil removed and installed. These time histories were obtained using the time marker signal from the rotor rig to initiate repetitive sampling of the radiated acoustic waveform. A 25 usec time increment between digital samples of the acoustic signature ensured adequate resolution of the waveform as the signal analyzer averaged 64 sequential time records. Polarity reversal of the acoustic signal due to the microphone signal conditioning electronics was accounted for by reversing the standard sign convention when labeling the vertical axes in Figure 26.

With the airfoil removed in Figure 26a, the sine wave like waveform represents the loading noise which dominated the spectrum in Figure 25a. Introducing the upstream airfoil (Fig. 26b) adds an impulsive signature to the measured waveform. Using the time marker from the rotor rig as a reference, the source of this impulsive noise was identified as the blade vortex interaction. This confirmed that higler harmonics in the spectrum of Figure $25 \mathrm{~b}$ represent the vortex interaction mechanism.

The above described differences between the loading and vortex interaction pressure signatures help to distinguish the two noise mechanisms. A further isolation is needed, however, if experimental results are to be compared with the present theory which models only the vortex interaction mechanism. Thus a procedure was developed to remove the loading noise contribution from the time waveform. The method is described in detail in Appendix B.

Figures $26 \mathrm{c}$ and $26 \mathrm{~d}$ show the resulting ensemble averaged blade vortex interaction noise signature for a single vortex intersection. (Note that smaller sampling increments $(5 \mu \mathrm{sec})$ were used for the high resolution time history in Figure 26d). The acoustic response consists of a positive overpressure, followed by a negative overpressure, and a second positive overpressure. The origin of this unsteady acoustic pressure signature was the angle of attack excursion sensed by each rotor blade as it intersected the vortex velocity field. As required by the acoustic noise model, the unsteady pressure is zero prior to and following the vortex intersection. 
Importance of Axial Mean Velocity Defect - The above discussion does not address the potential noise generated by the rotor blades intersecting the axial velocity defects in the vortex viscous core and the two dimensional wake region. Flow measurements indicated these additional sources of unsteady upwash existed in the present experiment. The magnitude of their contribution to the measured acoustic spectrum therefore required assessment. This was achieved by repeating each acoustic measurement with the semispan airfoil at $\alpha_{2}=0^{\circ}$. Under this condition the axial velocity defects are approximately the same but the vortex azimuthal velocity component is zero.

The spectrum in Figure 25 demonstrates that with the airfoil at $\alpha_{2}=0^{\circ}$, the higher harmonics generated by the blade vortex interaction are significantiy weaker when compared to the $\alpha_{2}=12^{\circ}$ spectrum. In general, the difference between $\alpha_{2}=0^{\circ}$ and $\alpha_{2}=12^{\circ}$ varied from 5 to $10 \mathrm{~dB}$ for all test conditions in the present study. This suggested that the measured acoustic response was dominated by the vortex azimuthal velocity component, $V_{\theta}$. It was, however, recognized that the airfoil wake defect could change as $\alpha_{2}$ changed from $12^{\circ}$ to $0^{\circ}$ in the above described spectrum comparison. Thus, the two dimensional wake region could not be totally eliminated as a potential noise source. However, additional experimental results confirming the dominance of the vortex intersection over the two dimensional wake is preser.ted in the subsection titled Vortex Intersection Angle Dependence. In this case, the upstream airfoil angle of attack (and consequently, the vortex strength) remained constant while the vortex intersection angle was changed at the rotor disk.

It should be noted that the symbol for the $\alpha_{2}=0^{\circ}$ data in Figure 26 consists of a circle combined with a dashed line. The circle is used to identify the tone amplitudes while the dashed line defines the broadband noise level between tones. The absence of a dashed line indicates broadband levels remained essentially unchanged. For those regions of the acoustic spectrum where the decibel difference between the $\alpha_{2}=0^{\circ}$ and $\alpha_{2}=12^{\circ}$ spectra is small (for example, at $4 \mathrm{kHz}$ in Figure 25b) experimental assessment of the theory was not attempted.

Importance of Incident Turbulence Noise - Unsteady velocity field measurements inside the vortex core suggested that incident turbulence noise could, potentially, contribute to the measured acoustic spectra. Based on the length scale shown in Figure 23, eddies in the vortex could be chopped multiple times resulting in narrowband random peaks at multiples of blade passing frequency. Similarly, turbulent eddies in the two dimensional wake could be chopped multiple times. Without an evaluation of this potential additional noise source the origin of the discrete tones in Figure $25 \mathrm{~b}$ would be unknown.

One method for identifying the presence of turbulence ingestion noise employs a high resolution narrowband frequency analysis. Using this approach randomness in the turbulent eddy structure is observed to cause broadening of the discrete tones generated by multiple intersections of the eddy. 
Figure 27 shows a high resolution spectrum of selected tones appearing in Figure 25b. At large blade passing frequencies such as $7 \times \mathrm{BPF}$ or $13 \times \mathrm{PBF}$, the apparent discrete tones in Figure 25b are shown to be narrowband random peaks. Although this observation suggests the existence of incident turbulence noise, several features must be carefully considered. First tone broadening due to multiple intersections with a turbulent eddy results in equal broadening at all blade passing harmonics as reported by Hanson (ref. 29). In contrast, the spectrum bandwidth in Figure 27 increases with frequency. Furthermore, Figure 22 of reference 29 predicts approximately 30 eddy intersections are needed to generate a $10 \mathrm{~Hz}$ bandwidth at the $10 \mathrm{~dB}$ down point in Figure 27. This exceeds, by a factor of 10, the number of eddy intersections calculated from Figure 23 . Finally, a $0.82 \mathrm{~m}$ axial length eddy would be required to generate the 30 intersections based on a freestream convection speed of $9 \mathrm{~m} / \mathrm{sec}$. Since the vortex core is on $\perp 0.008 \mathrm{~m}$ indiameter, the eddy length-to-diameter ratio would be approximately 100 which is unrealistic. In summary, the physical constraints imposed by the eddy axial length scale invalidates incident turbulence as a significant noise source in the present study. Similar arguments and conclusions apply for the turbulence in the two dimensional wake.

In the absence of turbulence ingestion noise it remains to establish the source of the narrowband random peaks in Figure 27. Noting the increase in spectrum bandwidth as tone frequency increased, it was conjectured that small variations in rotor speed broadened the tones generated by the vortex interaction mechanism. This possibility was evaluated by examining the spectrum of the rotor shaft time marker signal for similar broadening. Figure 28 shows the high resolution spectrum for the same center frequencies presented in the acoustic spectrum of Figure 27. For example, the $28 \mathrm{th}$ shaft harmonic corresponds to the 7 th blade passing harmonic for the four bladed rotor. A comparison of Figures 27 and 28 shows that the spectrum bandwidth, and even details of the spectrum shape, are the same. This establishes that small variations in the rotor speed (approximately $\pm 0.2 \mathrm{~Hz}$ based on Figure 28) created the narrowband random peaks observed in the acoustic spectrum.

Importance of Filter Bandwidth in Comparison of Experimental Data With Theoretical Predictions - Before the above described narrowband random measurements can be used for comparison with theory, the issue of filter bandwidth must be addressed. This is necessary since the present vortex interaction theory predicts only the acoustic energy generated at each blade passing harmonic. In essence, the theory predicts the total area under the distributed spectrum shape in Figure 27 . Thus, comparison of measured and predicted data requires careful consideration. Fortunately, all data used for assessment of the present theory was obtained using a $12.5 \mathrm{~Hz}$ bandwidth. This bandwidth encompasses the narrowband random peaks at all frequencies in Figure 25. Filter bandwidth, therefore, was not a limitation when comparing theory and experiment. 
It is recognized that the tone broadening due to variations in the rotor rotation speed can be eliminated electronically. This requires using a commericial spectrum tracking unit in conjunction with the spectrum analyzer. Such a unit was not available during the acoustic data reduction phase of the present study. The above assessment of the filter bandwidth, however, establishes that the processed data can be used without introducing errors.

The only exception to direct comparison of measured and predicted spectra arises when the narrowband tone (or even a discrete tone) spans two filter bands. This can occur irrespective of the use of a spectrum tracking unit. In this case, the measured power spectral density in adjacent bands must be added to give the total acoustic energy. Without this the tone amplitude can be $3 \mathrm{~dB}$ low if the tone is centered between filter bands. This may be the source of irregularities observed in the spectrum shape in the present study.

Effect of Rotor Speed Variations on Acoustic Signatures - In the absence of a spectrum tracking unit, the effect of a varying rotor rotation speed on the ensemble averaged acoustic signature must be assessed. For the 79 RPS test condition shown in Figure 26, the shaft frequency varied $\pm 0.2 \mathrm{~Hz}$. This variation is expected to occur over many revolutions since the rotor hub, shaft, and drive motor inertia damp the dynamic response of the system.

Using a conservative assumption that the rotation speed change occurs over one revolution, the rotation period would be compressed or expanded by $32 \mu s e c$. The impulsive signature in Figure $26 \mathrm{c}$ would then be shifted relative to the once per revolution time marker signal resulting in potential smearing of the ensemble averaged time waveform. Assuming the $\pm 32 \mathrm{usec}$ change develops linearly over one revolution, this corresponds to an $\pm 8 \mu \mathrm{sec}$ compression or expansion of each $1 / 4$ period. Potentially, the first impulse in Figure 26c, which is enlarged in Figure 26d, will be shifted \pm 8 usec relative to the time marker signal. The effect is negligible compared to the $500 \mu \mathrm{sec}$ duration of the acoustic impulsive signature.

Blade Tracking - Based on the similarity of the acoustic signatures in Figure $26 \mathrm{c}$ each blade generates the same unsteady lift due to vortex intersection. Small differences between blades are controlled by the loading noise waveform as shown in Figure 26a.

Blade Number Dependence

Approach - Figure 29 shows the effect of blade number, B, on the blade vortex interaction noise spectrum. The upper curve corresponds to the four bladed rotor while the lower curve was obtained for the two bladed configuration. Since the mean flow contraction changed with $B$, an adjustment in airfoil separation distance, $H$, was necessary to maintain a constant intersection station, $I$. This adjustment 
held $I / R_{1}$ constant while $B_{1}$ decreased from $14.6^{\circ}$ to $11.9^{\circ}$. Vortex strength, $r_{0} V_{0}$, changed only $3 \%$ based on the change in $H$ while vortex radius, $r_{0}$, remained essentially constant. All other parameters were identical as indicated in Table 1.

Note that the above described vortex properties are based on velocity field measurements with the rotor removed. Earlier discussions of the vortex velocity field, however, established that the rotor induced mean flow contraction did not change the vortex strength, $r_{0} V_{0}$, at the rotor plane. Also, it was shown that the contraction effect on $r_{0}$ can be calculated using the mean flow contraction ratio. (Resulting changes in acoustic radiation due to $r_{0}$ changes were, however, shown to be negligible in the present study.) These are important conclusions which are applied throughout the following experimental assessment of the acoustic theory. All assessments used the calculated vortex properties at the rotor plane as experimental inputs to the theory.

Results - The major effect observed in the comparisons of Figures 29a and 29b is a uniform decrease in noise level of approximately $7 \mathrm{~dB}$ as blade number changes from $B=4$ to $B=2$. To further quantify this result, differences between measured acoustic sound pressure levels are presented in Figure $29 \mathrm{c}$ at multiples of rotor shaft frequency. Here an average change of $7.5 \mathrm{~dB}$ is observed.

Present theory predicts a $6 \mathrm{~dB}$ change at all frequencies as blade number changes from $B=4$ to $B=2$. The larger measured change in Figure $29 \mathrm{c}$ was traced to the $1.7^{\circ}$ decrease of $B_{1}$ which also occurred as $B$ changed. Including this decrease in $\beta_{1}$, the predicted change is given by the solid line in Figure $29 \mathrm{c}$. The good agreement between theory and experiment is considered verification of the analysis considering the noise measurement accuracies and the variation in $B_{1}$. Figure 29c also verifies the well established 6 dB change associated with loading noise as blade number doubles. Experimental data at shaft harmonics given by $\mathrm{mB}=4$ and 8 represent the loading noise source.

It is important to note that the observer input angle, $\theta_{0}$, in the theoretical prediction for Figure $29 \mathrm{c}$ was increased by the blade pitch angle, $\alpha_{1}$. The rational for this correction was discussed earlier in the section titled Description of the Experiment (Subsection - Acoustic Measurements). This correction procedure is applied for all predictions presented in the remaining subsections.

\section{Vortex Strength Dependence}

Approach - Figure 30 shows the effect of vortex strength changes on blade vortex interaction noise. Here the upstream airfoil angle of attack was increased from $\alpha_{2}=6^{\circ}$ to $12^{\circ}$. Measured vortex strength, $r_{0} V_{0}$, was increased by a factor of two while vortex radius remained approximately constant. With the exception of the blade wake changes all other parameters were held constant. The wake changes, 
however, had a minimal effect on the noise generation as confirmed by the vortex intersection angle measurements described in the following subsection. This is because the vortex azimuthal velocity component dominated the acoustic response during the experiment.

Results - A comparison of Figures $30 \mathrm{a}$ and $30 \mathrm{~b}$ indicates vortex interaction tone amplitudes increase as vortex strength increases. Sensitivity of the noise to changes in vortex strength is defined quantitatively in Figure 30c where an average increase of $5.5 \mathrm{~dB}$ is observed at frequencies above the loading noise (represented by $\mathrm{mB}=4$ and 8 ). This increase agrees closely with the theoretically calculated change which is also presented in Figure 30c. The prediction is based on the measured values of vortex strength and vortex radius and is represented as a solid line in the figure.

The measured change in Figure $30 \mathrm{c}$ confirms several parametric features of the present analysis. First the unsteady blade upwash given by Equation 36 is a linear function of vortex strength $r_{0} V_{0}$. Doubling vortex strength, therefore, increases the acoustic intensity by $6 \mathrm{~dB}$ when Equation 36 is combined with Equation 8. Second, changing $r_{0} V_{0}$ in Equation 36 changes the spectrum uniformly since wavenumber and vortex strength appear as isolated parameters. Finally the linear dependence on $r_{0} V_{0}$ and the uniform effect on the acoustic spectrum applies irrespective of the blade vortex intersection angles $\theta_{\mathrm{V}}$ or $\phi_{\mathrm{V}}$. (This observation is based on examining Equations (36) and (40)). The above conclusions are important when considering changing the main rotor tip vortex properties or the vortex trajectory with the objective of reducing blade vortex interaction noise. These conclusions provide useful scaling procedures for guiding future designs.

\section{Vortex Intersection Angle Dependence}

Approach - Figures $31 \mathrm{a}$ and $31 \mathrm{~b}$ compare measured acoustic spectra for different vortex intersection angles, $\beta_{1}=14.6^{\circ}$ and $0^{\circ}$. The change in $\beta_{1}$ was achieved by decreasing blade number, $B$, and blade pitch angle, $\alpha_{1}$. These parametric changes eliminated the mean flow contraction, thereby, forcing $\beta_{1}$ to zero. A small change in separation height, $\mathrm{H}$, was also required to hold the blade vortex intersection station constant. The upstream airfoil angle of attack, however, remained fixed so that the two-dimensional wake defect and vortex axial velocity defect were essentially the same in Figures $31 \mathrm{a}$ and $31 \mathrm{~b}$.

The multiple parametric changes described above must be accounted for and are described here to document quantitatively the effect on Figure 31 . First, the earlier assessment of blade number dependence demonstrated a $6 \mathrm{~dB}$ reduction in sound pressure level as blade number changed from $B=4$ to 2 . Second, changes in $\mathrm{H}$ caused a $9 \%$ increase of vortex strength in Figure $31 \mathrm{~b}$. Based on Equations 36 and 8 , this results in a $0.7 \mathrm{~dB}$ increase of sound pressure level irrespective 
of changes in blade pitch. Finally, the $2.5^{\circ}$ decrease in blade pitch angle between Figures $31 \mathrm{a}$ and $31 \mathrm{~b}$ resulted in approximately a $1 \mathrm{~dB}$ reduction in sound pressure level. This is based on the independent assessment of pitch angle effects which showed a weak dependence on $\alpha_{1}$. In summary, blade vortex interaction tone amplitudes in Figure $31 \mathrm{~b}$ should be increased by $6.3 \mathrm{~dB}$ to achieve the same test condition as in Figure 3la. This result will be used shortly to interpret the significance of Figure 31 .

A similar discussion of test conditions and changes in parameters is required before Figure 32 can be interpreted. Here, vortex intersection angle changed from $B_{1}=14.6^{\circ}$ to $B_{1}=4.4^{\circ}$. Similar to Figure $31, \mathrm{~B}, \mathrm{H}$, and $\alpha_{1}$ were also changed. The acoustic responses to these changes essentially cancelled each other.

The above described experimental changes in vortex intersection angle are based on smoke flow visualization photographs with the angle $\beta_{1}$ referenced to the rotor blade. In contrast, ${ }^{\theta} \mathrm{V}$ is referenced to the normal to the blade planform. Angles $\beta_{1}$ and $\theta_{V}$ are equivalent only if $\alpha_{1}=0^{\circ}$. Since the present test program was conducted with finite blade pitch angles an analytical transformation was employed to convert the measured values for $\beta_{1}$ and $\gamma$ to blade referenced angles $\theta_{\mathrm{V}}$ and $\phi_{\mathrm{V}} \cdot$

Results - The $16 \mathrm{~dB}$ decrease due to changing $\beta_{1}$ between Figures $31 \mathrm{a}$ and $31 \mathrm{~b}$ (after incorporating a $6.3 \mathrm{~dB}$ correction to account for other parameters changing) verifies a fundamental result of the present acoustic theory. The analysis predicts that a vortex intersection normal to the blade planform does not generate noise. This is dramatically confirmed by the large reduction in sound pressure level in Figure 31b. The low sound pressure levels in Figure 31b also confirm that axial velocity defects did not significantly contribute to the tone noise generation in the present study. This is an important conclusion since it confirms that the vortex azimuthal velocity component dominated the acoustic response during the experiment.

A quantitative assessment of the sensitivity to vortex intersection angle is obtained by comparing the tone amplitudes in Figures $32 \mathrm{a}$ and $32 \mathrm{~b}$. Note that a finite intersection angle was employed in Figure $32 \mathrm{~b}$ to ensure that the vortex azimuthal velocity dominated over the axial velocity defects during the blade vortex interaction. Differences between tone amplitudes in these figures are presented as a function of shaft harmonic number in Figure 32c. The difference has been corrected for blade number but changes due to varying $H$ or $\alpha_{1}$ are not included. Theoretical predictions (corrected only for blade rumber effect) are also shown in Figure 32. The predictions are based on the experimental test conditions and include the measured vortex strength and $\alpha_{1}$. The difference between the predictions for Figures $32 \mathrm{a}$ and $32 \mathrm{~b}$ can, therefore, be compared directly with the experimentally determined differences in tone amplitudes shown in Figure 32c. The comparison of measured and predicted sensitivity due to changing $\beta_{1}$ shows reasonable agreement with theory. The observed change is considered verification of theory considering the accuracy of measuring $\beta_{1}$. 
It is interesting to note that the $\mathrm{dB}$ difference in Figure $32 \mathrm{c}$ approaches zero for shaft harmonic number $\mathrm{mB}=4$ and 8 . This is because these harmonics are dominated by loading noise as indicated from comparing the $\alpha_{2}=12^{\circ}$ and $0^{\circ}$ in Figures $32 \mathrm{a}$ and $32 \mathrm{~b}$.

Having confirmed the vortex intersection angle sensitivity, a simplified dependence on the angle $\theta_{V}$ can bc extracted from the general theory. Equation (36) indicates that when $\cos ^{2} \theta_{V}$ is approximately unity the unsteady upwash is controlled by the $\tan \theta_{\mathrm{V}}$ term. This dependence requires only that $\phi_{\mathrm{V}}$ remain constant and applies irrespective of the absolute value of $\phi_{\mathrm{V}}$ (a conclusion determined from combining Equations 40 and 36 ). The dependence of $\tan \theta_{\mathrm{V}}$ is also uniform over all wavenumbers, or in other words, over the complete acoustic spectrum.

To demonstrate the application of this scaling law consider estimating the change in Figure 32c. Initially, $\beta_{1}=14.6^{\circ}$ and $\gamma=0^{\circ}$ corresponds to blade referenced intersection angles given by $\theta_{\mathrm{V}}=17.4^{\circ}$ and $\phi_{\mathrm{V}}=57.6^{\circ}$. As the intersection angle changes to give $B_{1}=4.4^{\circ}$ and $0^{\circ}$, the blade referenced angles become $\theta_{\mathrm{V}}=8.3^{\circ}$ and $\phi_{\mathrm{V}}=32.3^{\circ}$. The estimated change in tone sound pressure level associated with these different vortex intersection conditions is $6.6 \mathrm{~dB}$. This value is comparable to the analytical prediction in Figure 32c. Note that the variation in $\phi_{V}$ could not be accounted for in the simple tan $\theta_{V}$ scaling law creating a difference between the predicted and estimated effect on the noise radiation.

\section{Blade Pitch Angle Dependence}

Approach - Blade pitch angle dependence was determined by operating the rotor blades at two pitch angles given by $\alpha_{1}=9.5^{\circ}$ and $14^{\circ}$. As in previous test cases, blade number and airfoil separation distance required adjustment to hold intersection angle, $\beta_{l}$, and intersection station, $I$, constant at the rotor plane. Angle $\beta_{1}$ was held constant within 1.8 degrees while all other parameters remained unchanged.

Results - A comparison of acoustic spectra in Figures 33a and $33 \mathrm{~b}$ documents the effect of changing blade pitch angle. Quantitative assessment of the change is given in Figure $33 c$ as a function of shaft harmonic number. A general observation is that blade vortex interaction noise is not significantly influenced by the small changes in $\alpha_{1}$. Figure $33 c$ also shows the theoretically predicted change in tone level as pitch angle changes. (The prediction includes the small change in $\beta_{1}$ to permit direct comparison with the experiment.) Based on the comparison with experiment, it is concluded that the theory adequately predicts the effect of pitch angle change.

The analytically predicted effect of changing pitch angle can also be estimated from the previously identified $\tan \theta_{V}$ parameter dependence. Changing $\alpha_{1}$ by rotating the airfoil in Figure 4 through small angles about the $y$ axis causes $\theta_{V}$ to change 
while $\phi_{y}$ remains approximately constant. Considering the intersection angles in Figure 33a, $\alpha_{1}=9.5^{\circ}, \beta_{1}=5^{\circ}$, and $\gamma=0^{\circ}$ correspond to $\theta_{\mathrm{V}}=10.7^{\circ}$ and $\phi_{\mathrm{V}}=27.9^{\circ}$. In Figure $33 \mathrm{~b}, \alpha_{1}=14^{\circ}, \beta_{1}=6.8^{\circ}, \gamma=0^{\circ}$ corresponds to $\theta_{\mathrm{V}}=15.5^{\circ}$ and $\phi_{\mathrm{V}}=26.2^{\circ}$. Based on the simplified $\tan \theta_{\mathrm{v}}$ dependence the increase in sound pressure level due to increasing $\alpha_{1}$ is estimated at $3.3 \mathrm{~dB}$. This agrees with the experimental result and the predicted curve obtained from the computer coded version of the theory. Note also that changes in blade pitch angle influence the blade vortex interaction noise spectrum uniformly for small values of $\theta_{\mathrm{V}}$.

Mach Number Dependence

Approach - Sensitivity of blade vortex interaction noise to tip Mach number changes was assessed by varying $\mathrm{M}_{\mathrm{T}}$ from 0.35 to 0.55 . Blade number was also changed while the associated change in $\beta_{I}$ was limited to $1.2^{\circ}$. The remaining parameters were held constant.

Results - The effect of increasing blade tip Mach number by $60 \%$ is demonstrated by comparing the measured spectra in Figures $34 \mathrm{a}$ and $34 \mathrm{~b}$. Differences between tone amplitudes in these figures are presented in Figure $34 \mathrm{c}$. Note that data is presented only at shaft harmonics for which a 5 dB difference existed between the $\alpha_{2}=12^{\circ}$ and $0^{\circ}$ specta. Recall that the $\alpha_{2}=0^{\circ}$ spectra establish the contribution of the axial velocity defect to the radiated noise.

The solid line in Figure $34 \mathrm{c}$ represents (including the small change in $B_{1}$ ) the theoretically calculated change in sound pressure level due to increasing $\mathrm{M}_{\mathrm{T}} \cdot{ }^{1} \mathrm{~A}$ $4 \mathrm{~dB}$ difference between theory and experiment is observed at low frequencies but agreement improves at high frequencies. A decrease in the predicted curve with increasing frequency is attributed to the frequency dependence of the airfoil response function.

\section{Directivity Pattern}

Approach - Figure 35 shows measured vortex interaction acoustic spectra at three radiation angles. Spectra, such as these, were used to determine the source directivity pattern. Measurements were limited to a range of $\theta_{0}=60^{\circ}$ to $140^{\circ}$ due to the upstream and downstream anechoic chamber walls. In addition, the rotor rig support stand interferred with noise radiated to angles larger than $\theta_{0}=140^{\circ}$. All parameters were held constant in this evaluation.

Directivity Pattern Based on Acoustic Spectra - For frequencies associated with blade vortex interaction noise ( $\mathrm{f}>3 \mathrm{x} \mathrm{BPF}$ ), tone noise spectra in Figure 35 exhibit a directivity pattern which is nearly symetric about the rotor plane. A further evaluation of the vortex interaction noise directivity pattern is provided by Figure 36. Here measured tone levels are plotted as a function of $\theta_{0}$ for 1ncreasing 
blade passing frequencies. Loading noise dominated BPF, $2 \times \mathrm{BPF}$, and $3 \times \mathrm{BPF}$. Consequently, the directivity associated with the vortex interaction mechanism could not be investigated at these frequencies. However, at higher blade passing frequencies experimental evaluation was possible. In this case, the measured radiation pattems were found to be approximately symmetric about the plane of the rotor blade which corresponded to $e_{0}=80.5^{\circ}$.

Predicted directivity patterns are also shown in Figure 36 for selected blade passing frequencies dominated by blade vortex interaction noise ( $5 \mathrm{x} \mathrm{BPF}$, $8 \times \mathrm{BPF}, 11 \times \mathrm{BPF}$ ). Good agreement between theory and experiment exists for the directivity pattern shape. In addition, the measured and predicted minima occur near the plane of the blade. Since the acoustic source dipole axis is aligned normal to the blade planform in the present analysis, the source radiation is zero in the plane of the blade as verified by the experiment.

Although generally good agreement between experiment and theory was observed for directivity pattern shape large differences occurred in the absolute tone amplitude. These differences were due to a general decrease in predicted tone amplitude with increasing frequency for all radiation angles, $e_{0}$. This observation is based on a comparison of measured and predicted acoustic spectra which is discussed later in the subsection titled Assessment of Acoustic Spectrum and Pressure Signature.

It should be noted that the value of $\theta_{0}$ used for the input to the analytical prediction was increased by the value of $\alpha_{1}$. This is due to the difference in the reference axes selected for the theoretical and experimental definition of $\theta_{0}$ (see subsection titled Acoustic Measurements). According to Figure 4, $\theta_{0}$ in the analysis is measured from the normal to the blade planform. In contrast, the experimental program referenced $\theta_{0}$ to the rotor centerline. At the blade vortex intersection station, these angles differed by the blade pitch angle setting. Comparison between theory and experiment, therefore, required increasing $\theta_{0}$ by $\alpha_{1}=9.5^{\circ}$ in the predictions. This approach is used consistently throughout the report.

Directional Radiation Characteristics of Blade Vortex Interaction Impulsive Signature - The previous discussion of directivity pattern focused on tone amplitude changes occurring over the range of $\theta_{0}=60^{\circ}$ to $140^{\circ}$. Additional directivity features of blade vortex interaction noise can be identified from acoustic pressure signatures. Figure 37 shows ensemble averaged waveforms measured at selected radiation angles $\theta_{0}=60^{\circ}, 90^{\circ}, 120^{\circ}$ and $140^{\circ}$. The data corresponds to the same test condition described in Figure 36.

Waveforms in Figure 37 contain the combined loading and blade vortex interaction signatures. Dominance of loading noise is evident in the sine wave character of the time history at all radiation angles. In contrast the impulsive signature peaks at angles outside the plane of rotation but diminishes to zero at $\theta_{0}=90^{\circ}$ which is close to the plane of the rotor blade. These time history traces provide additional explanations for the directivity features determined from acoustic spectra. 
Blade vortex interaction acoustic signatures are further isolated in Figure 38 . Here measured waveforms are shown for the same microphone angles shown in Figure 37. Loading noise has been removed using the procedure described in Appendix B. Several waveform features are noteworthy. First, waveform shape is observed to be antisymmetric about the plane of rotation. Second, peak amplitude decreases near the plane of rotation. Finally, absolute waveform amplitudes are equal on opposite sides of the rotor. These important characteristics confirm the dipole acoustic source model developed in the present study.

\section{Assessment of Acoustic Spectrum and Pressure Signature}

Objective - Previous discussions focused on the experimental assessinent of the sensitivity of blade vortex interaction noise to isolated changes of each parameter in the acoustic theory. Conclusions obtained from this assessment confirmed that the theory models the parametric dependence of each individual parameter. It now remains to determine if the analysis predicts the measured acoustic spectrum shape and absolute sound pressure levels. In addition, the analysis will be tested to determine if the measured acoustic pressure signatures can be predicted.

Acoustic Spectrum - Figure 39 shows measured and predicted spectra for Test Condition B. Before comparing these spectra, the presence of steady loading noise must be discussed since this mechanism dominated frequencies given by $B P F, 2 \times B P F$, and $3 \times$ BPF. This additional noise source was removed from the measured spectrum by first using the waveform subtraction technique described in Appendix B.1. A Fast Fourier Transform (FFT) of the resulting isolated blade vortex interaction time history (consisting of 13 consecutive vortex interactions in the continuous time waveform) provided the acoustic spectrum shown in Figure 39. This spectrum coincides with the spectrum in Figure $25 \mathrm{t}$ except for the BPF tone and the first two harmonics.

The general conclusion from the spectrum comparison in Figure 39 is that tone amplitudes are predicted to within $5 \mathrm{~dB}$ at low frequencies. At high frequencies, the present analysis significantly underpredicts the data.

Acoustic Signatures - Figure 40 a shows the predicted acoustic signature for the experimental test case presented in Figure 38c. Both waveforms are plotted on the same scales and correspond to the same measurement angles. A comparison of the measured and predicted waveforms indicates that the duration of the acoustic signature is similar as is the sign of the maximum peak pressure. In addition, the experimentally observed antisymmetric waveform occurring on opposite sides of the rotor are predicted by the present analysis. Calculated waveforms were found to be identical (not shown) at $\theta_{0}=50^{\circ}$ and $120^{\circ}$, except for the sign reversal in the acoustic pressure. (Note that these angles are symetric around the plane of the rotor blade in the present experiment.) The qualitative agreement between theory and experiment again conforms the dipole acoustic source model. 
While the general features of the blade vortex interaction signature are predicted, details cannot presently be calculated. For example, the peak amplitude, triangular shape, and combined positive and negative peaks of Figure $38 \mathrm{c}$ are not predicted.

Assessment of Difference Between Theory and Experiment - The rotor blade interaction with the two dimensional wake of the upstream airfoil was initially considered to be a possible source of the difference between theory and experiment. This mechanism was, however, evaluated experimentally during the blade vortex intersection angle tests. The results indicated that when $\beta_{1}=0$, the axial velocity defects did not significantly contribute to the tone noise generation. This conclusion confirmed that the vortex azimuthal velocity component dominated the acoustic response during the present study.

A second possible source of the difference between theory and experiment is the infinite span airfoil assumption used in the analysis. With this assumption, physical constraints imposed by the finite span rotor blade are not satisfied by the theory. To understand the consequences of the infinite span airfoil model, recall that the far field sound is related to the time derivative of the airfoil loading. Calculating the area under the far field pressure waveform is, therefore, equivalent to a time integral of the pressure signature in Figure 40a. The result of the integral is the difference between the lift when the airfoil is at an infinite distance upstream and downstream of the airfoil

$$
\operatorname{AREA}=\int_{-\infty}^{\infty} \dot{L} d t=L_{\infty}-L_{-\infty}
$$

For the present infinite span airfoil model and the $1 / \mathrm{r}$ decay of the azimuthal velocity vector, Equation 42 gives a finite or non-zero difference between $L_{\infty}$ and $L_{-\infty}$. This occurs because the calculated pressure pulse in Figure 40 is always less than zero. Pressure peaks of opposite sign, as in the experiment, would cause the area in Equation 41 to approach zero.

The non-zero value obtained from Equation 42 represents a theory limitation since a finite span airfoil situated at an infinite distance frotn the vortex has a zero value for the quantity $\mathrm{L}_{\infty}-\mathrm{L}_{-\infty}$. To approximate the finite span blade tested in the present experiment, Equation 22 was multiplied by an exponential decay function defined as $\exp \left[-\left(\mathrm{R}_{\mathrm{O}} / \mathrm{r}\right)^{2}\right]$. Here $\mathrm{R}_{\mathrm{o}}$ represents the radius at which the exponential amplitude is $e^{-1}$. The rapid decay of the vortex velocity field due to the additional exponential decay function ensures that the integral in Equation 42 has a zero value. Introducing the exponential decay function represents more than 
a mathematical termination of the influence of the vortex velocity field. It is also an approximate simulation of a finite span airfoil. The acoustic signature is the same whether the vortex velocity field is terminated or the airfoil is terminated as in the case of a finite span. This equivalence, however, assumes that the blade end effects can be ignored.

Using the exponential decay function to model the finite span blade results in predicted acoustic waveforms which display some of the features of the measured waveforms. This is illustrated in Figure $40 \mathrm{~b}$ where predicted pressure signals are presented for a range of $R_{0} / c$ values where $c$ corresponds to the blade chord. Here $R_{0} / c=1$ approximates the distance, $I$, in the present experiment, from the vortex interaction station to the blade tip. Terminating the analytical infinite span airfoil model at this distance generated an acoustic pressure signal which exhibits combined positive and negative peaks similar to the experiment. The $R_{\mathrm{O}} / \mathrm{c}=1$ curve displays an initial positive pressure pulse, a negative peak, and a small positive pressure waveform which returns to zero as time increases. Increasing $R_{0} / c$ causes the acoustic pressure to return to the one-sided waveform .predicted in Figure 40a.

The above discussion illustrated how the waveform shape depends on the infinite span airfoil assumption. This assumption can also result in incorrect predictions of the amplitude of the acoustic signature as illustrated by the following example. Consider a plane normal to the span line of the airfoil. Also let the normal to the airfoil planform and the vorticity vector be in this normal plane $\left(\phi_{\mathrm{V}}=0^{\circ}\right.$ or $180^{\circ}$ and $\theta_{\mathrm{V}}$ arbitrary). Then the unsteady transverse flow sensed by the airfoil will be antisymmetric about this normal plane; i.e., if the flow is upward on one side of this normal plane it is downward on the opposite side with an equal magnitude. An observer stationed anywhere in the normal plane will measure zero sound since a positive pressure perturbation produced on one side of the normal plane is cancelled by the opposing negative pressure.

If the infinite span airfoil in the above description is now truncated on one side of the normal plane (as in the case of the finite span rotor blade used in the present experiment) the opposing unsteady pressure responses no longer occur and the above described acoustic cancellation disappears. This finite span effect is not accounted for by the previously described experimental decay factor since the factor retains the symmetry of pressure response on the airfoil surface. Thus, it is possible to also achieve an increase in predicted sound level when using a truncated (finite span) airfoil.

The asymmetry associated with the finite span provides a potential explanation for the discrepancy between predicted and measured acoustic spectra (Fig. 39) in addition to acoustic signatures (Fig. 40). This follows since the present microphone measurement plane almost coincided with the above described normal plane. Also, for the small values of $\beta_{1}$ tested in the present experiment, the vorticity vector was almost in the normal plane. 


\section{CONCLUSIONS}

1. Ingestion of a vortex by a rotor generates harmonic noise which extends to high frequencies. Acoustic pressure signatures associated with this noise mechanism display impulsive waveforms superimposed on the blade loading acoustic pressure signals.

2. Linear dependence of measured blade vortex interaction noise on blade number and vortex strength was closely predicted by the present first principles acoustic theory. The absence of noise for vortex trajectories normal to the blade was also predicted. For oblique intersections with a blade, calculated changes in acoustic radiation due to intersection angle modifications were within 2 decibels of the measured data. A simplified scaling law was determined for estimating these changes at small intersection angles.

3. The predicted low sensitivity to blade pitch angle modifications for a typical range of rotor operating conditions was cnnfirmed experimentally. The strong noise dependence on local Mach number was calculated although the sensitivity to Mach number change was overpredicted at low frequencies.

4. Measured blade vortex interaction directivity patterns confirmed the theoretical dipole acoustic source model at all frequencies. The minimum sound pressure level observed experimentally in the plane of the blade was predicted analytically.

5. Absolute levels for acoustic spectra and directivity patterns were predicted, without the use of empirical or adjustable constants, to within 5 decibels at low frequencies. At high frequencies, sound pressure levels are currently underpredicted.

6. General features of the measured blade vortex interaction signature were predicted. Antisymmetry of the measured waveform on opposite sides of the rotor plane confirmed the theoretical dipole directivity model. Duration of the impulsive acoustic signature in addition to the sign of the maximum pressure peak was predicted. Detailed features of the measured acoustic signature, such as peak amplitude, triangular shape, and combined positive and negative peaks, are not presently calculated. 
APPENDIX A

Vortex Velocity Field Measurement Technique

Decomposition of Total Velocity Vector - The azimuthal $\left(\mathrm{V}_{\theta}\right)$ and axial (U) velocity components of the vortex velocity field are shown in. Figure 9. Decomposition of the measured voltage from the single slanted hot film into $V_{\theta}$ and $U$ employs the following procedure.

First, it is necessary to recognize that the probe sensing element can be rotated until it lies in the plane formed by the components $V_{\theta}$ and $U$. (Note that the vortex radial velocity is assumed to be zero in this velocity decomposition approach.) This unique orientation is illustrated in Figure $A-1$. Here the $V_{\theta}$ and $\mathrm{U}$ components lie in the plane formed by the hot film sensor and the support prongs. In Figure 9 this corresponds to alignment of the probe sensing plane with a plane parallel to the $z, y$ axes. In this case, the probe sensing plane is normal to a radial line penetrating the vortex center.

To decompose the total velocity vector, $\mathrm{U}_{\mathrm{T}}$, in Figure $\mathrm{A}-1$ into the corresponding values of $\mathrm{V}_{\theta}$ requires two separate measurements. These correspond to two orientations which are $180^{\circ}$ apart in Figure $\mathrm{A}-1$. The velocity components are then given by the equations:

$$
\begin{aligned}
\mathrm{V}_{\theta} & =\mathrm{U}_{\mathrm{T}} \cos \left(\beta_{\mathrm{F}}-\mathrm{a}_{1}\right)=\mathrm{U}_{\mathrm{T}}\left(\cos \beta_{\mathrm{F}} \cos \mathrm{a}_{1}+\sin \beta_{\mathrm{F}} \sin \mathrm{a}_{1}\right) \\
\mathrm{U} & =\mathrm{U}_{\mathrm{T}} \sin \left(\beta_{\mathrm{F}}-\mathrm{a}_{1}\right)=\mathrm{U}_{\mathrm{T}}\left(\sin \beta_{\mathrm{F}} \cos \mathrm{a}_{1}-\cos \beta_{\mathrm{F}} \sin \mathrm{a}_{1}\right)
\end{aligned}
$$

Representing the two separate normal velocity components at each wire orientation as $\mathrm{U}_{\mathrm{N} 1}$ and $\mathrm{U}_{\mathrm{N} 2}$ gives

$$
\begin{aligned}
& \mathrm{U}_{\mathrm{N} 1}=\mathrm{U}_{\mathrm{T}} \cos \mathrm{a}_{1} \\
& \mathrm{U}_{\mathrm{N} 2}=\mathrm{U}_{\mathrm{T}} \sin \mathrm{a}_{1}
\end{aligned}
$$

Since the sensor mean voltage output $E_{1}$ and $E_{2}$ at each of the two orientations is linearly related to the normal velocity component

$$
\begin{aligned}
& \mathrm{U}_{\mathrm{N} 1}=\mathrm{KE}_{1} \\
& \mathrm{U}_{\mathrm{N} 2}=\mathrm{KE}_{2}
\end{aligned}
$$

where $k$ represents the velocity versus voltage calibration coefficient. 
Using. Equations $A-5$ and A-6 in the expressions for $V_{\theta}$ and $U$ gives the sum and difference expressions for velocities measured using a standard crosswire

$$
\begin{aligned}
v_{\theta} & =0.707 \mathrm{~K}\left(\mathrm{E}_{1}-\mathrm{E}_{2}\right) \\
\mathrm{U} & =0.707 \mathrm{~K}\left(\mathrm{E}_{1}+\mathrm{E}_{2}\right)
\end{aligned}
$$

Thus, a measurement at two orientations in a plane normal to a radial line penetrating the vortex center gives $V_{\theta}$ and $U$.

Instead of rotating the sensor at each radial velocity station to obtain $\mathrm{V}_{\theta}$ and $U$ the following procedure was used. A continuous traverse was conducted on a radial line through vortex center for each of the two orientations. An analog output from the traverse system was used to drive one axis of a two directional plotter. Two continuous traces of the voltages $E_{1}$ and $E_{2}$ were then obtained as the sensor penetrated the vortex. Graphically summing and differencing the plotter traces decomposed the measured voltages into $E_{1}-E_{2}$ and $E_{1}+E_{2}$ at each radial station. A simple application of the calibration factor $\mathrm{K}$ then permitted changing the plotter axes notation to $V_{\theta}$ and $U$ as shown in Figure 17.

Identification of the Vortex Center - The above described radial velocity traverse assumes a knowledge of the line penetrating the vortex center. The following simple procedure was developed to identify the vortex center.

As described in the previous subsection, there exists an alignment of the sensor measuring plane for which $V_{\theta}$ and $U$ are easily determined. This plane is normal to the radial line through the vortex center. The plane can be identified by rotating the sensor $360^{\circ}$ about the probe shaft. When the sensor voltage output reaches a maximum, all velocity components lie in the plane of the sensor. This orientation is designated in Figure $A-1$ as the maximum voltage output orientation. Other orientations can be shown to give smaller voltage values with a minimum occurring at $180^{\circ}$ from the maximum orientation.

The vortex center then lies on a line perpendicular to the unique measurement plane. Rotating the sensor at numerous stations on the perimeter of the vortex provided a series of radial lines whose intersection point defines the vortex center. Figure A-2 provides a schematic description of this experimental procedure. A digital angular position encoder with a $0.33^{\circ}$ resolution was used to sense the probe orientation. 


\author{
APPENDIX B \\ Experimental Technique for Isolating Blade-Vortex \\ Interaction Impulsive Signatures
}

Measured acoustic pressure signatures contained both loading noise and the blade-vortex interaction impulsive signature. The following procedure was employed to isolate the impulsive waveform permitting direct comparison of measured and predicted blade-vortex interaction pressure signatures.

The experimental technique begins with the ensemble averaged waveform for the combined loading and vortex interaction noise. A sample time history is shown in Figure B-la where the time marker signal from the rotor rig is used to initiate repetitive sampling of the acoustic waveform. This waveform is obtained with the upstream semispan airfoil at a finite angle of attack $\left(\alpha_{2}=12^{\circ}\right.$ in the present example).

A second waveform is obtained with the airfoil at $\alpha_{2}=0^{\circ}$ (Fig. B-1b). Under this condition, the vortex strength is forced to zero while the loading noise remains the same. At the same time, nolse generated by intersecting the axial velocity defect in the vortex core and in the two dimensional wake are considered to remain the same. The difference between Figures $B-1 \mathrm{a}$ and $\mathrm{B}-1 \mathrm{~b}$ represents the isolated blade vortex interaction signature which is shown in Figure B-1d. An enlarged version of this waveform is presented in Figure B-le. The above described ensemble averaged pressure signatures and the arithmetic subtraction of these waveforms were obtained on a dual channel Fast Fourier Transform spectrum analyzer with time domain averaging capability.

The rationale for using the $\alpha_{2}=0^{\circ}$ waveform is based on the need to account for the axial velocity defect which is also present in the waveform of Figure B-la. The noise generated by this velocity defect creates the small pressure pulse observed in Figure $\mathrm{B}-\mathrm{lb}$. Using the loading noise pressure signature with the upstream airfoil removed (Fig. B-1c) would fail to account for this noise mechanism. 


\section{REFERENCES}

1. Leverton, J. Y., J. C. Pollard, and C. R. Wills: Main Rotor Wake/Tail Rotor Interaction. Vertica, Vol. 1, No. 3, pp. 213-221, 1977.

2. Pegg, R. J., and P. A. Shidler: Exploratory Wind Tunnel Investigation of the Effect of the Main Rotor Wake on Tail Rotor Noise. Helicopter Acoustics, NASA CP-2052, Part 1, pP. 205-219, 1978.

3. Paterson, R. W., R. K. Amiet: Noise of a Model Helicopter Rotor Due to Ingestion of Turbulence. NASA CR-3213, November 1979.

4. Amiet, R. K.: Aerodynamic Sound Production and the Method of Matched Asymptotic Expansions. Ph.D. Thesis, Cornell University, 1969.

5. Widnall, S.: Helicopter Noise Due to Blade-Vortex Interaction. J. Acoustical Society of America, Vol. 50, pp. 354-365, 1971.

6. Widnall, S. E. and T. L. Wolf: Effect of Tip Vortex Structure on Helicopter Noise Due to Blade-Vortex Interaction. J. Aircraft, Vol. 17, pp. 705-711, 1980 .

7. Adamczyk, J. J.: The Passage of an Infinite Swept Airfoil Through an Oblique Gust. NASA CR-2395, 1974.

8. von Karman, T. and W. R. Sears: Airfoil Theory for Nonuniform Motion. J. of the Aeronautical Sciences, Vol. 8, pp. 104-108, 1940.

9. Graham, J. M. R.: Similarity Rules for Thin Airfoils in Non-Stationary Flows. J. Fluid Mech., Vol. 43, pp. 753-766, 1970.

10. Amiet, R. K.: Compressibility Effects in Unsteady Thin-Airfoil Theory. AIAA Journal, Vol. 12, pp. 253-255, 1974.

11. Amiet, R. K.: Effects of Compressibility in Unsteady Airfoil Lift Theories. Unsteady Aerodynamics, University of Arizona/AFOSR Symp., Tucson (Ed., R. B. Kinney), pp. 631-653, 1975.

12. Amiet, R. K.: High Frequency Thin-Airfoil Theory for Subsonic Flows. AIAA Journal, Vol. 14, pp. 1076-1082, 1976.

13. Amiet, R. K.: Low Frequency Approximations in Unsteady Small Perturbation Subsonic Flows. J. Fluid Mec., Vol. 75, pp. 545-552, 1976. 
14. Amiet, R. K.: Airfoil Response to an Incompressible Skewed Gust of Small Spanwise Wavenumber. AIAA Journa1, Vol. 14, pp. 541-542, 1976.

15. Graham, J. M. R.: Lifting Surface Theory for the Problem of an Arbitrarily Yawed Sinusoidal Gust Incident on a Thin Aoerfoil in Incompressible Flow. Aero. Quart., Vo1. 21, pp. 182-198, 1970.

16. Georges, T. M.: Acoustic Ray Paths through a Model Vortex with a Viscous Core. J. Acoust. Soc. Amer., Vol. 51, pp. 206-209, 1972.

17. Larson, R. S, and K. W. Robbins: Refraction of Sound by Aircraft Wing Tip Vortices. AIAA Paper \#80-0975, 1980.

18. Morse, P. M. and K. U. Ingard: Theoretical Acoustics. McGraw Hill Book Co., New York, 1968, Equation 11.2.33.

19. Landah1, M.: Unsteady Transonic Flow. Pergamon Press, New York, 1961.

20. Gradshteyn, I. S., and I. M. Ryzhik: Tables of Integrals Series and Products. Academic Press, New York, 1965.

21. Abramowitz, M. and I. A. Stegun: Handbook of Mathematical Functions. Dover Publications, Inc., New York, 1968.

22. Newland, D. E.: An Introduction to Random Vibrations and Spectral Analysis, Longman, London, 1975.

23. Cooley, J. W., P. A. W. Lewis, and P. D. Welch: The Fast Fourier Transofrm and its Applications. IEEE Transactions on Eductions, Vol. 12, pp. 27-34, 1969.

24. Paterson, R. W., P. G. Vogt, and W. M. Foley: Design and Development of the United Aircraft Research Laboratories Acoustic Research Tunnel. J. of Aircraft, Vo1. 10, No. 7, pp. 427-433, 1973.

25. Hama, F. R.: An Efficient Tripping Device. J. of Aeronautical Sciences, Vol. 24, pp. 236-237, March 1957.

26. Schlinker, R. H., and R. K. Amiet: Shear Layer Refraction and Scattering of Sound. AIAA Paper No. 80-0973, 1980. 


\section{REFERENCES (Cont' $d$ )}

27. Prandt1, L.: Attaining a Steady Air Stream in Wind Tunnels. NACA TM 726, 1933.

28. Batchelor, G. K.: An Introduction to Fluid Dynamics. Cambridge University Press, 1976.

29. Hanson, D. B.: Spectrum of Rotor Noise Caused by Atmospheric Turbulence. $\mathrm{J}$. of the Acoustical Society of America, Vol. 56, No. 1, pp. 110-126, 1974. 


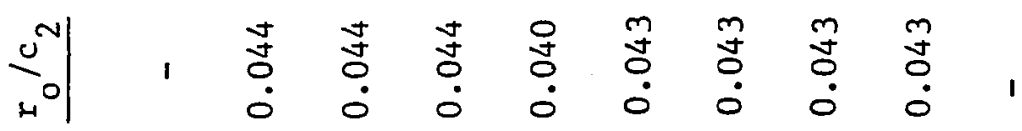

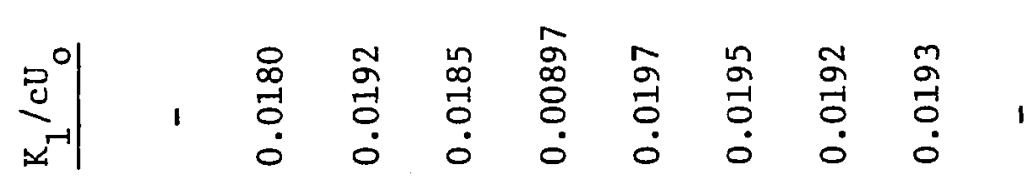

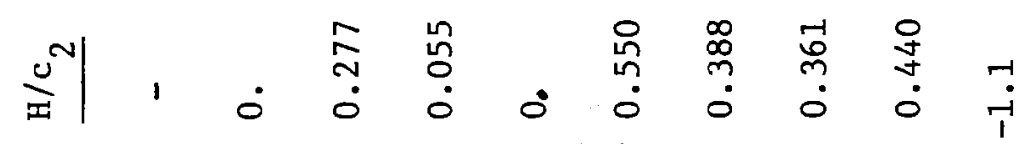

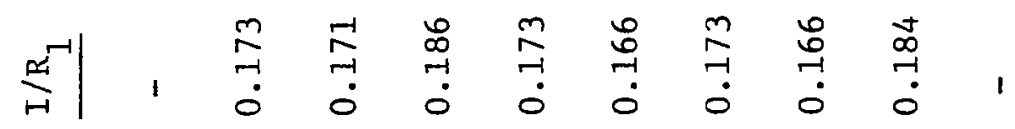

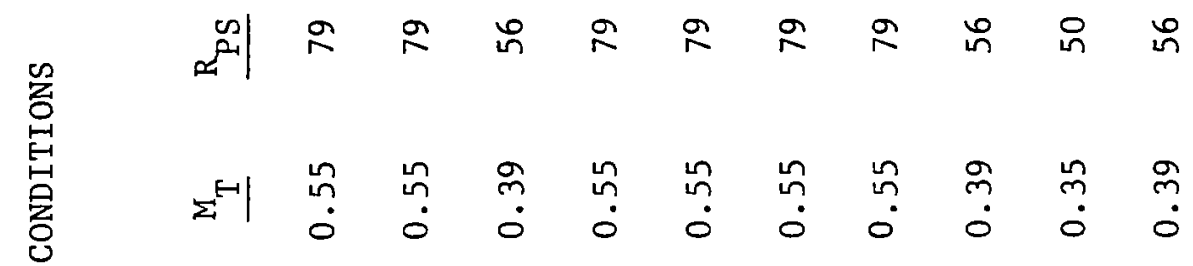

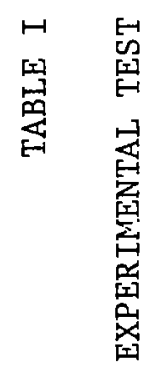

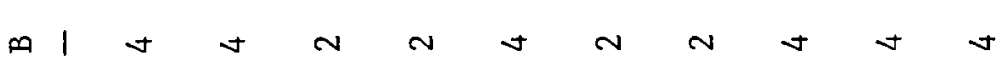

車,

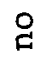

N

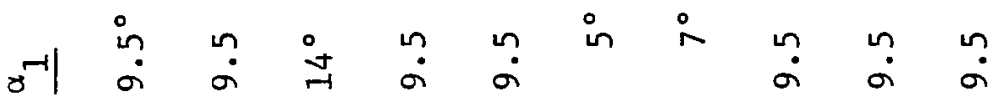

\& 


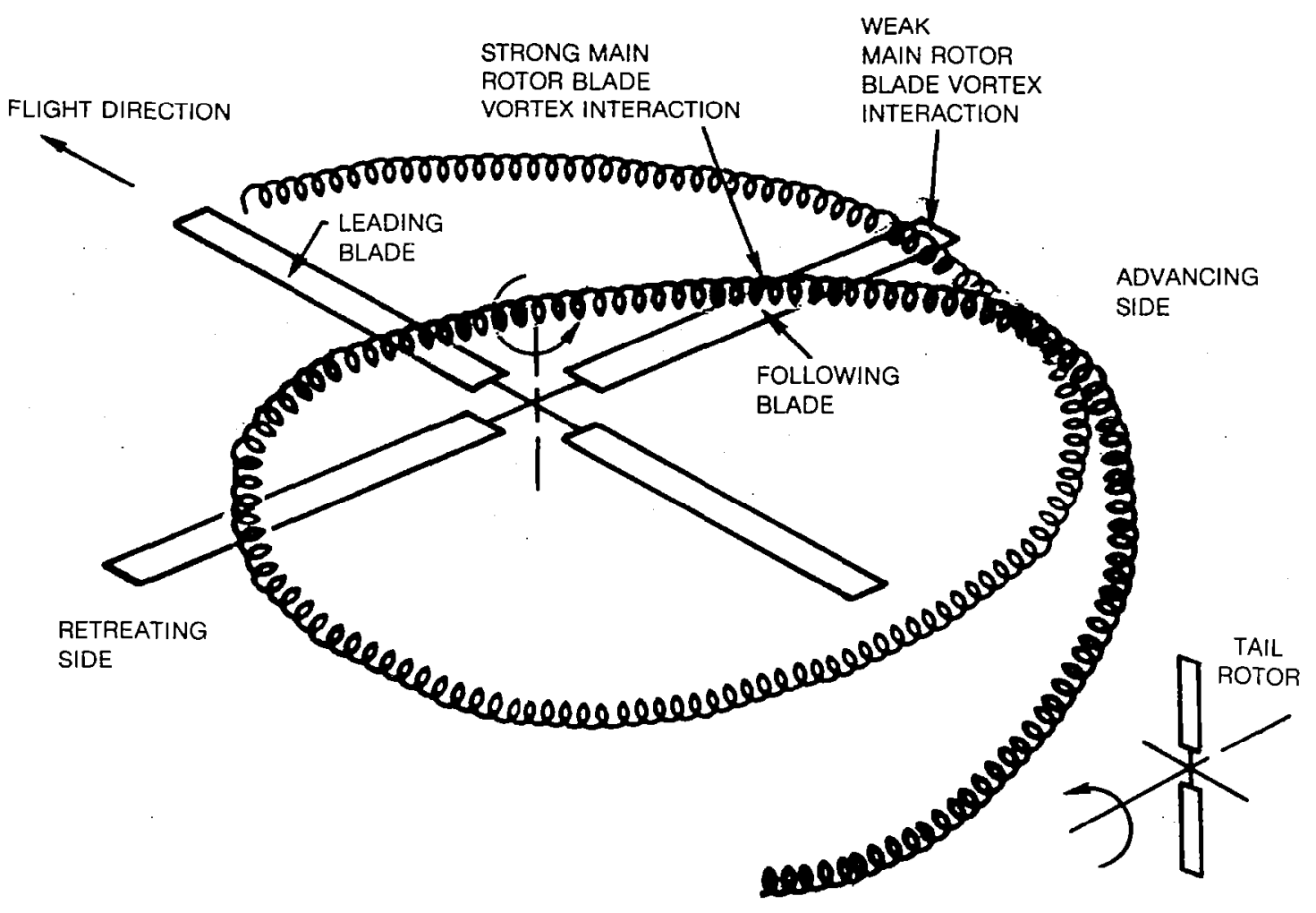

a) ILLUSTRATION OF MAIN ROTOR AND TAIL ROTOR BLADE VORTEX INTERACTION

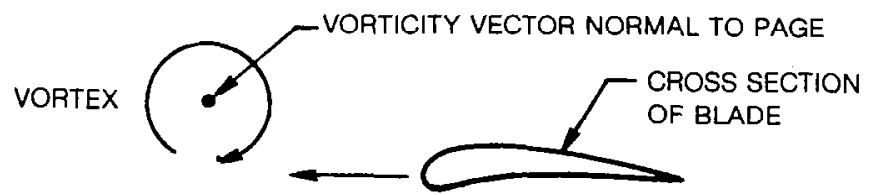

b) ILLUSTRATION OF MAIN ROTOR BLADE VORTEX INTERACTION WITH VORTEX PARALLEL TO LEADING EDGE

Figure 1 - Helicopter Blade Vortex Interactions 


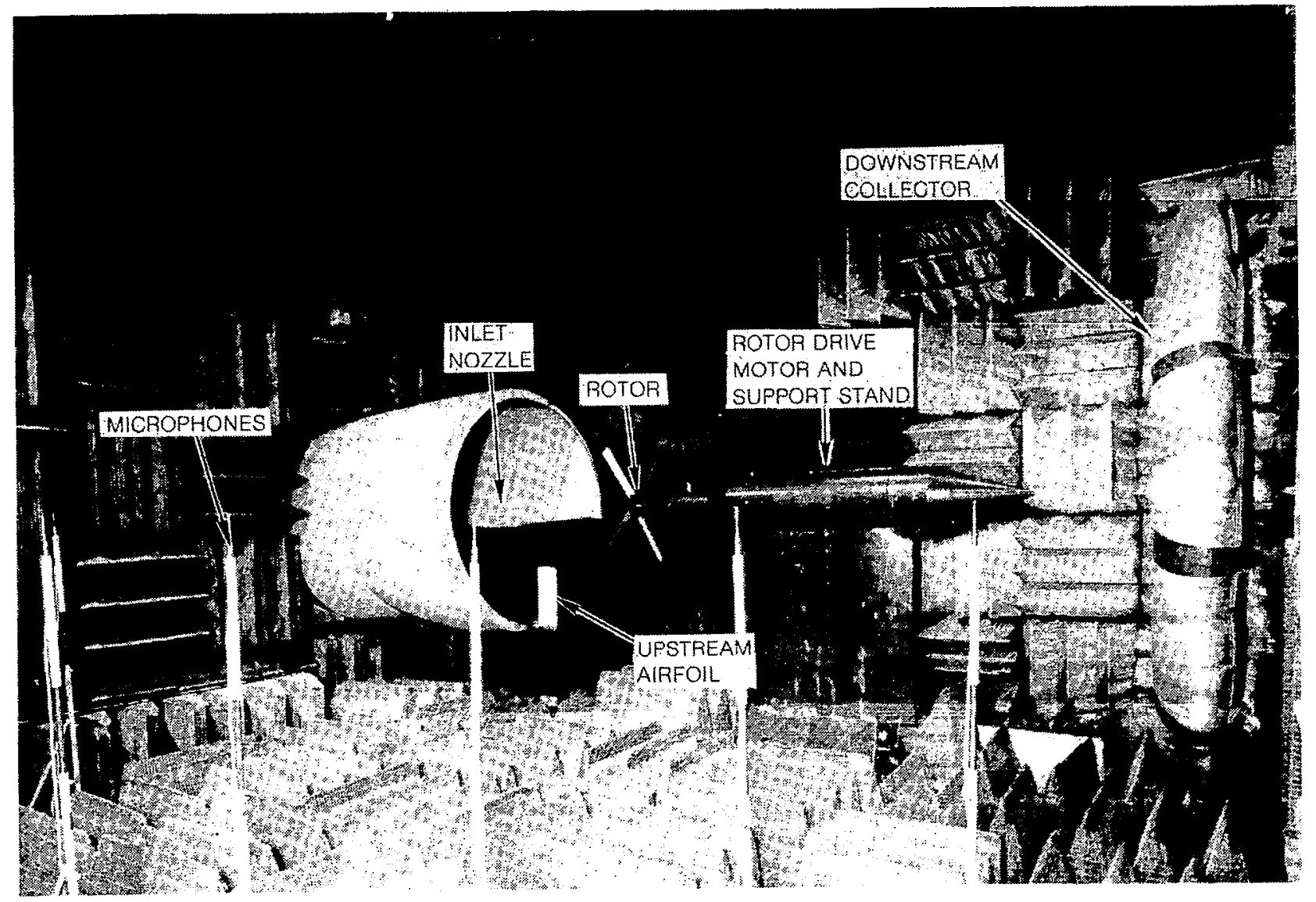

Figure 2 - Open Jet Test Section Arrangement in Anechoic Chamber 


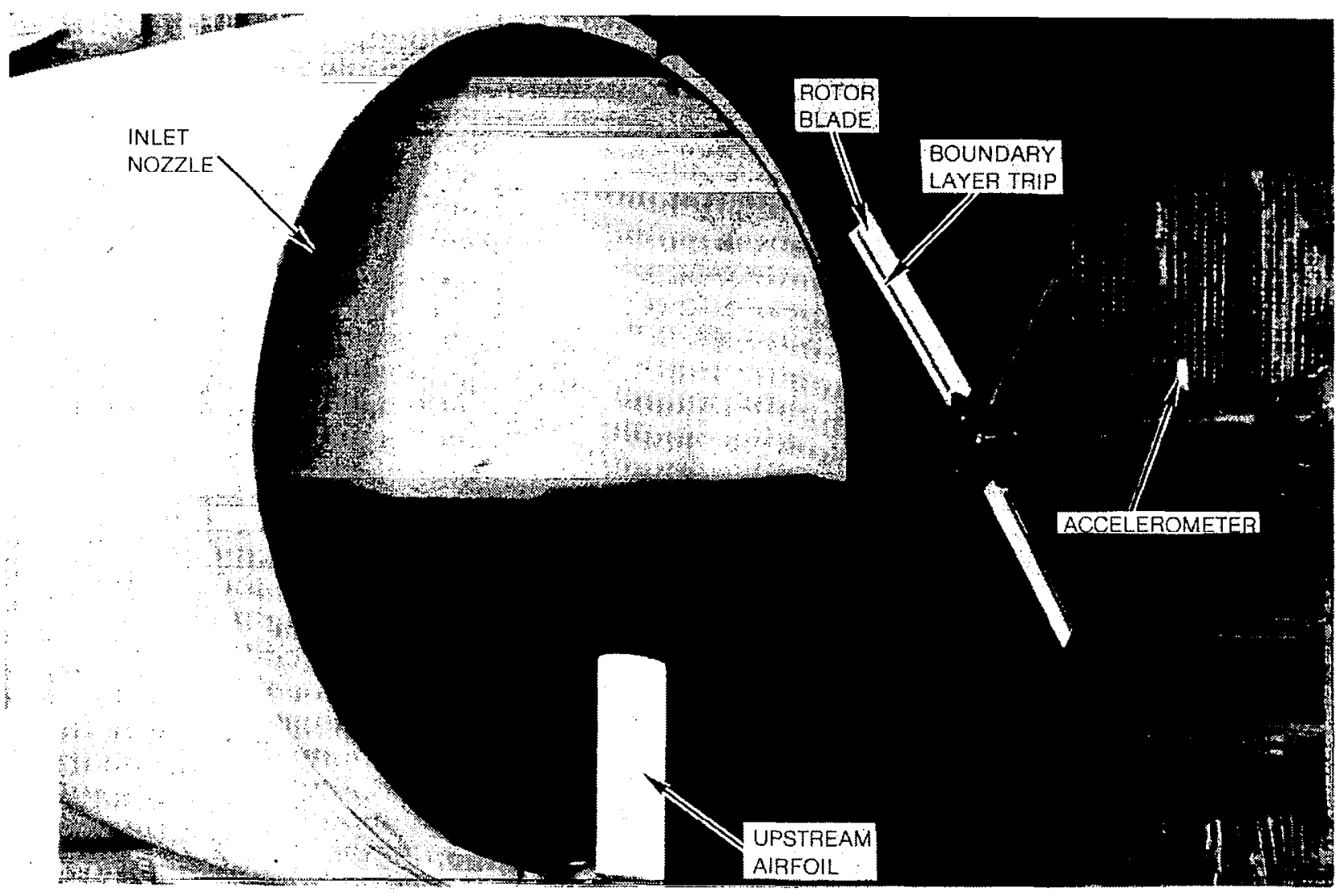

Figure 3 - Upstream Model Airfoil and Rotor Blade with Boundary Layer Trip 

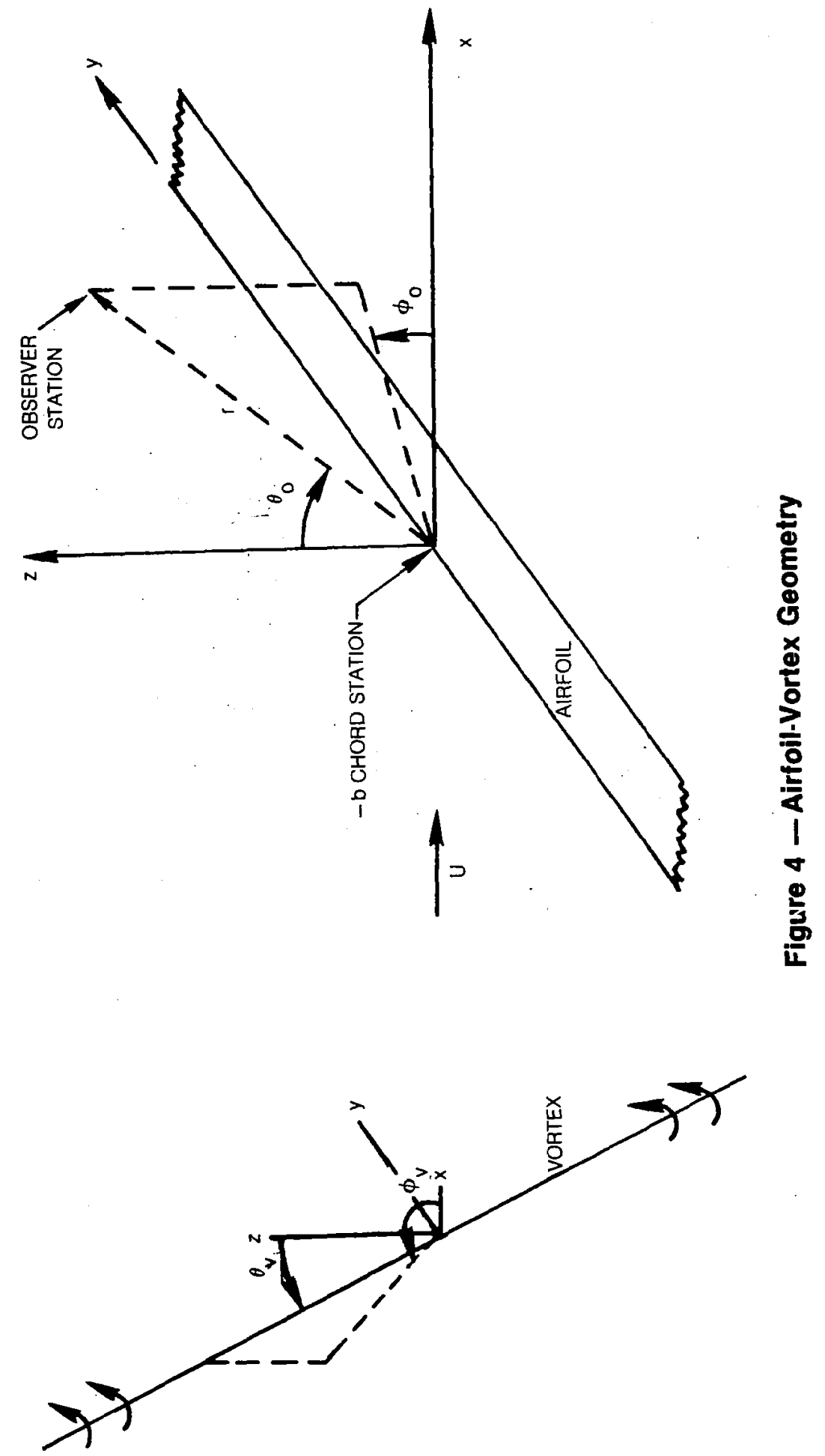

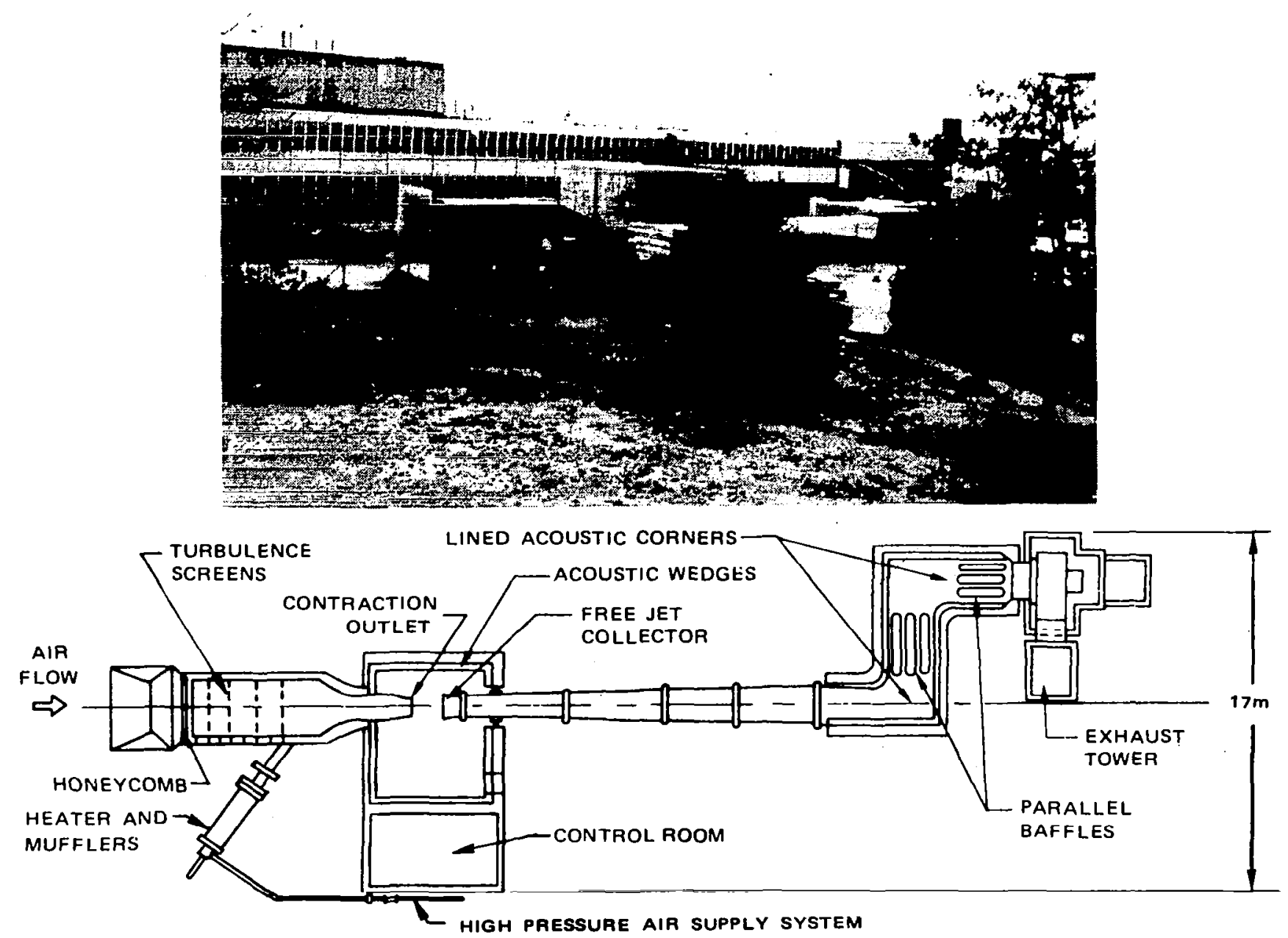

TOP VIEW

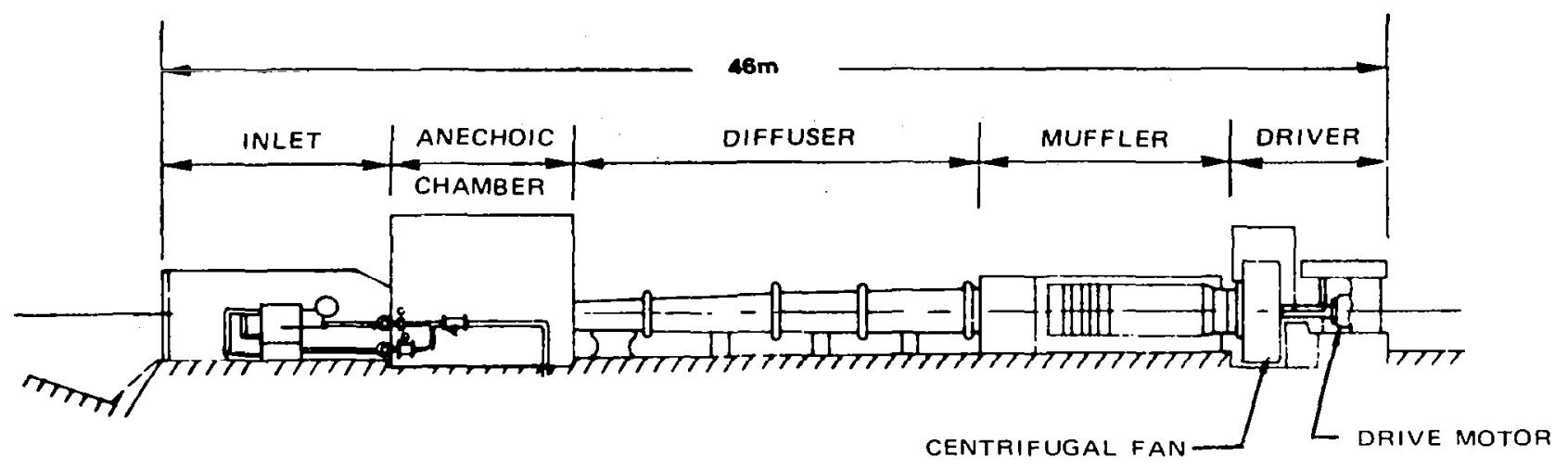

SIDE VIEW

Figure 5 - UTRC Acoustic Research Tunnel 


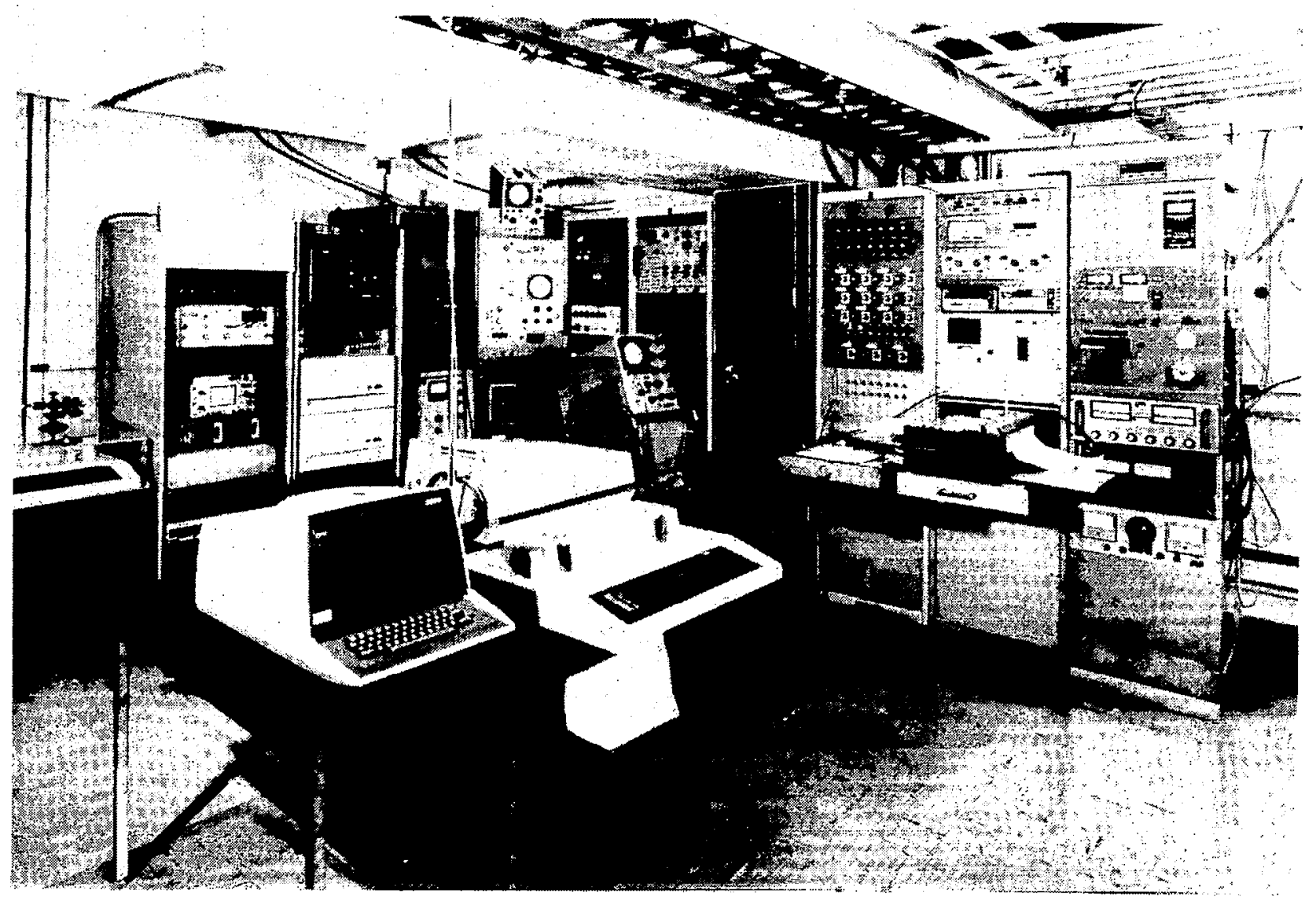

Figure 6 - Acoustic Research Tunnel Control Room with Data Acquisition and Data Reduction Instrumentation 


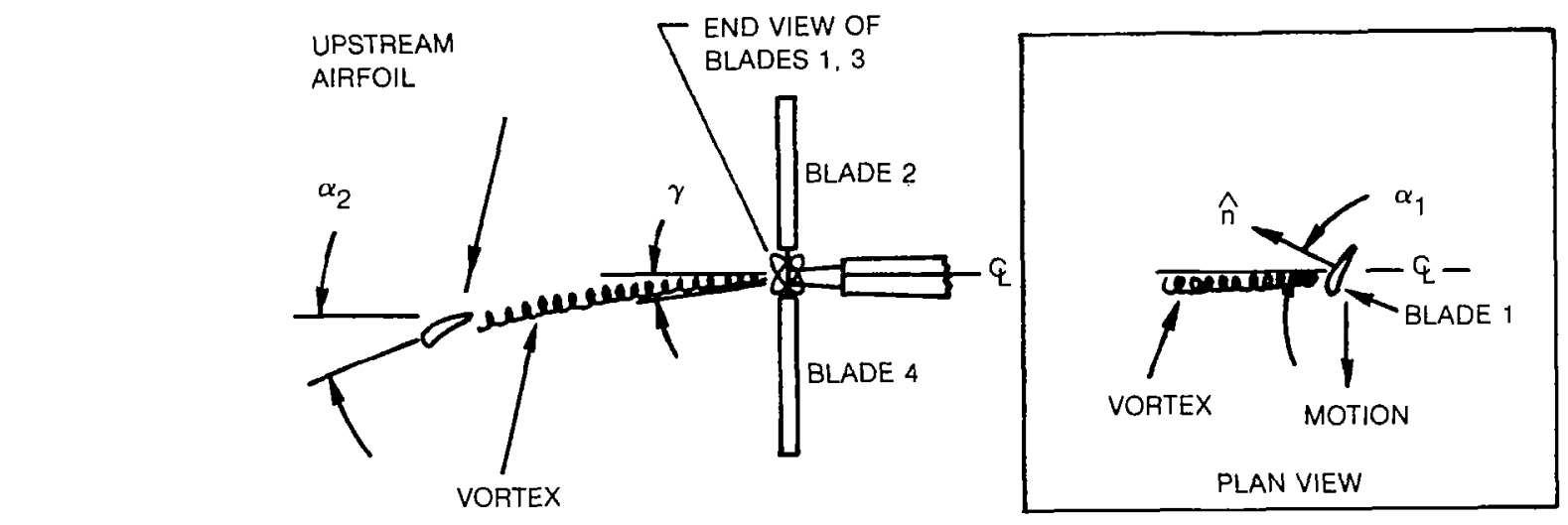

a) PLAN VIEW WITH UPSTREAM AIRFOIL AND ROTOR BLADES

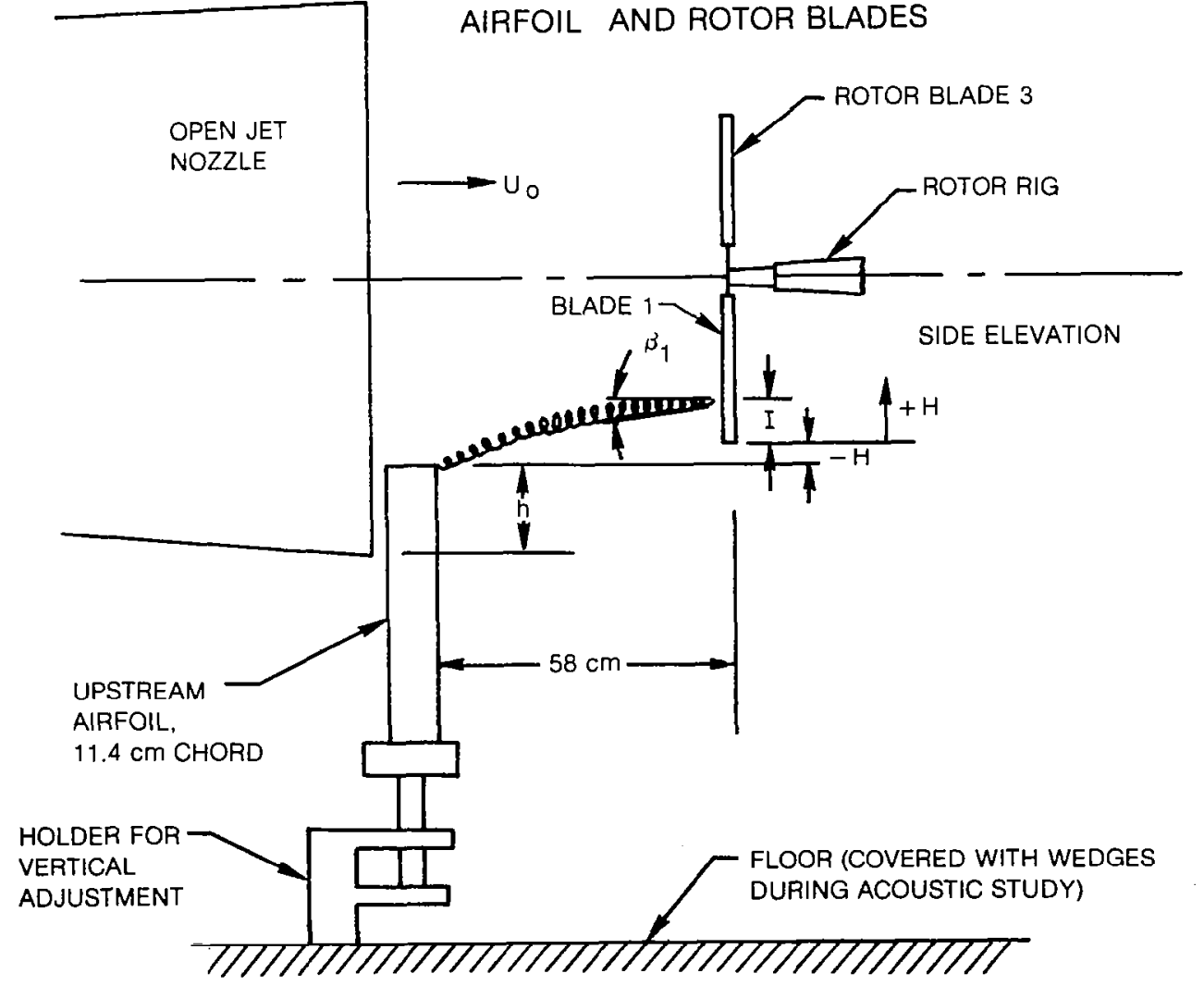

b) SIDE VIEW SHOWING OPEN JET TEST FACILITY

Figure 7 - Detailed Schematic of Vortex Generator and Model Rotor 


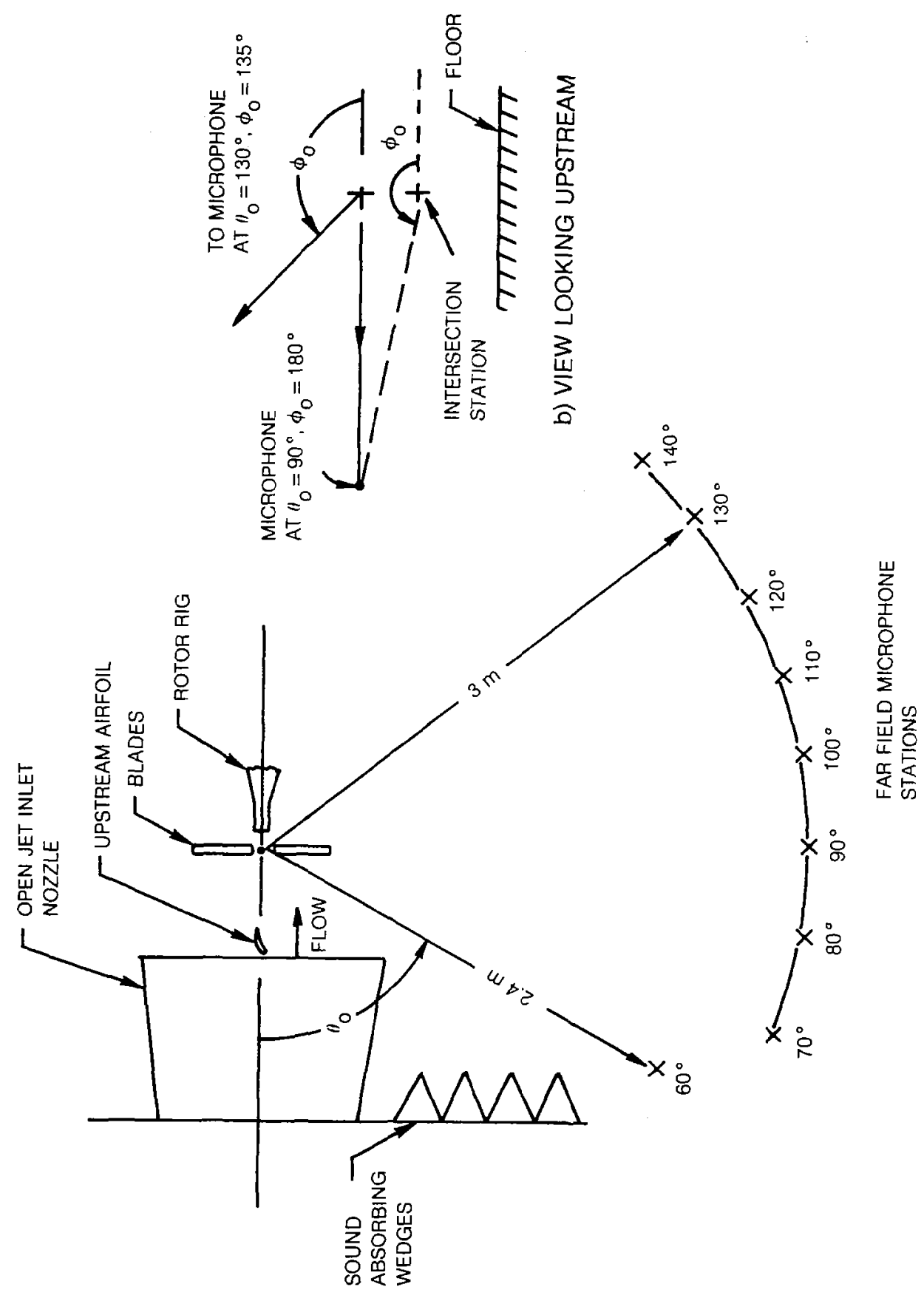

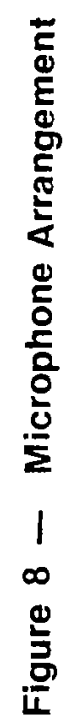




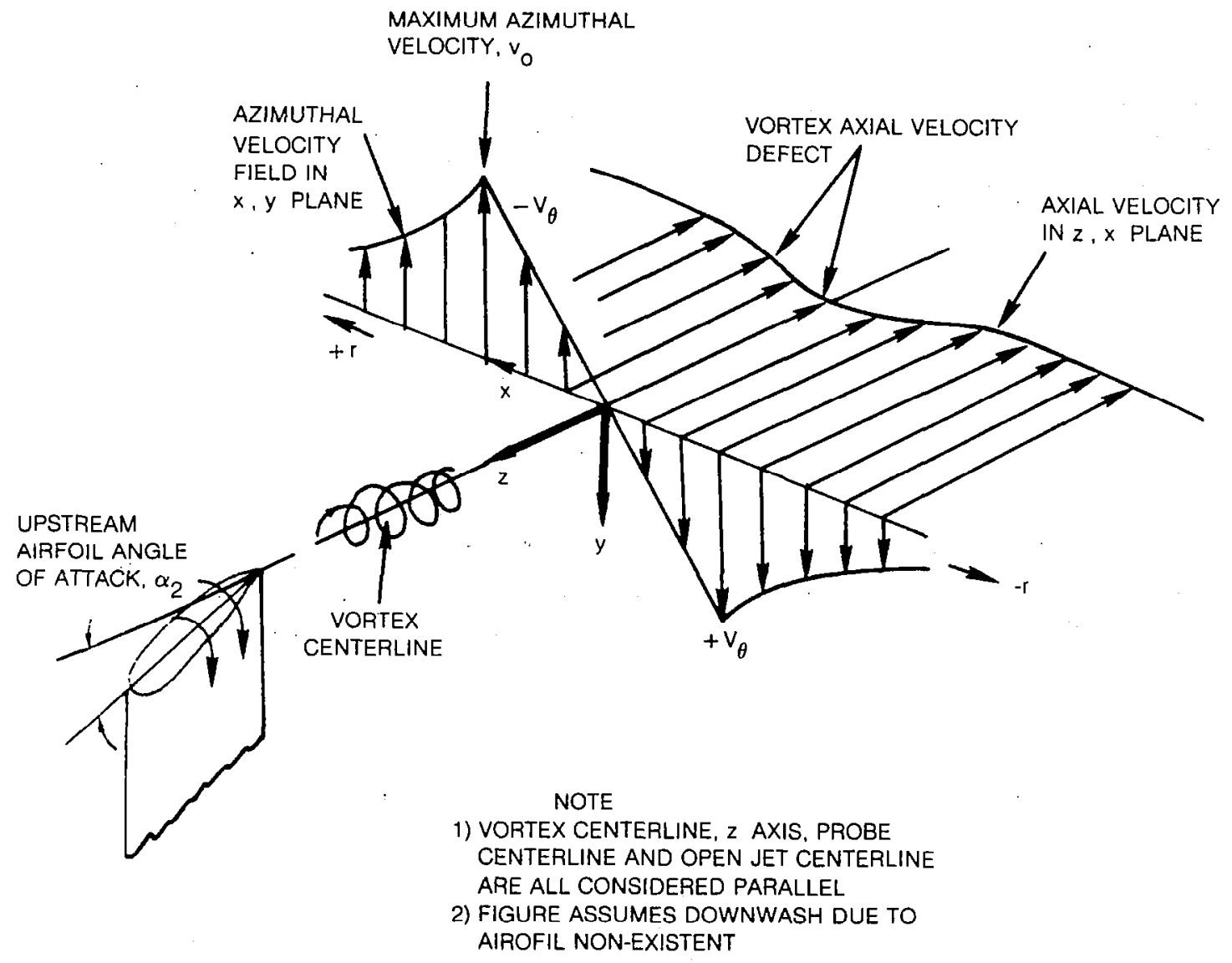

Figure 9 - Definition of Vortex Velocity Field 


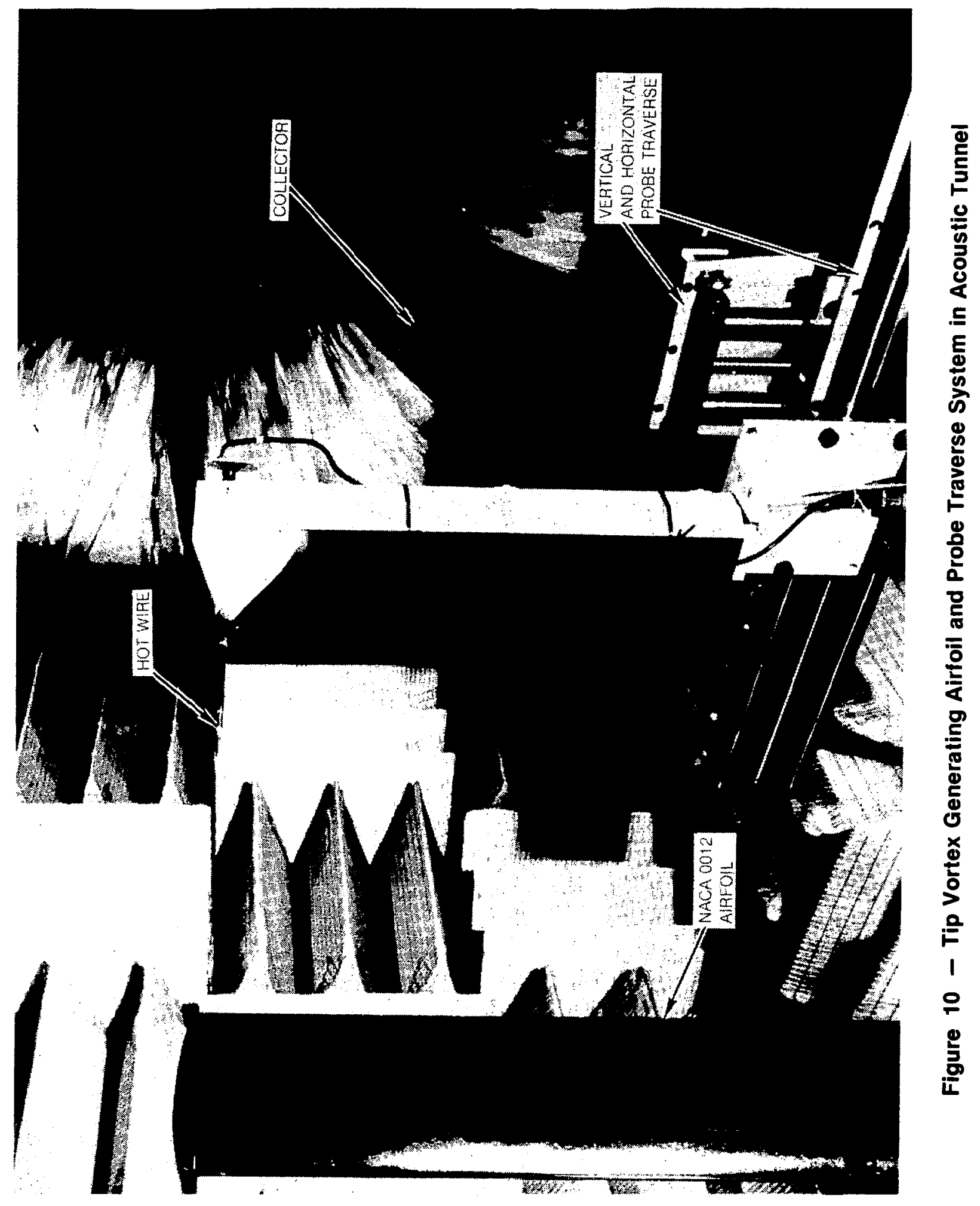



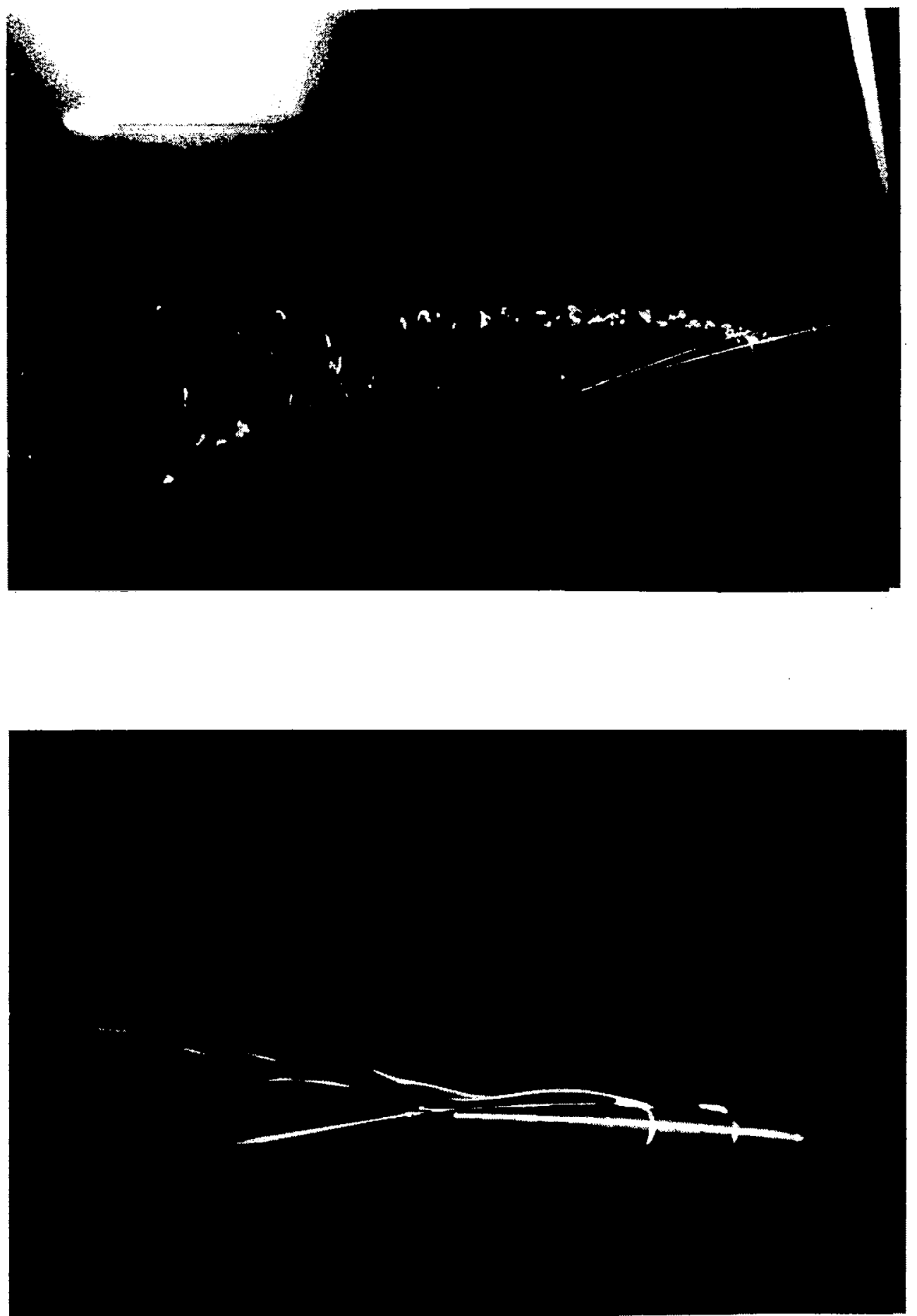

Figure 11 - Smoke Flow Visualization Showing Tip Vortex for Two Operating Conditions 


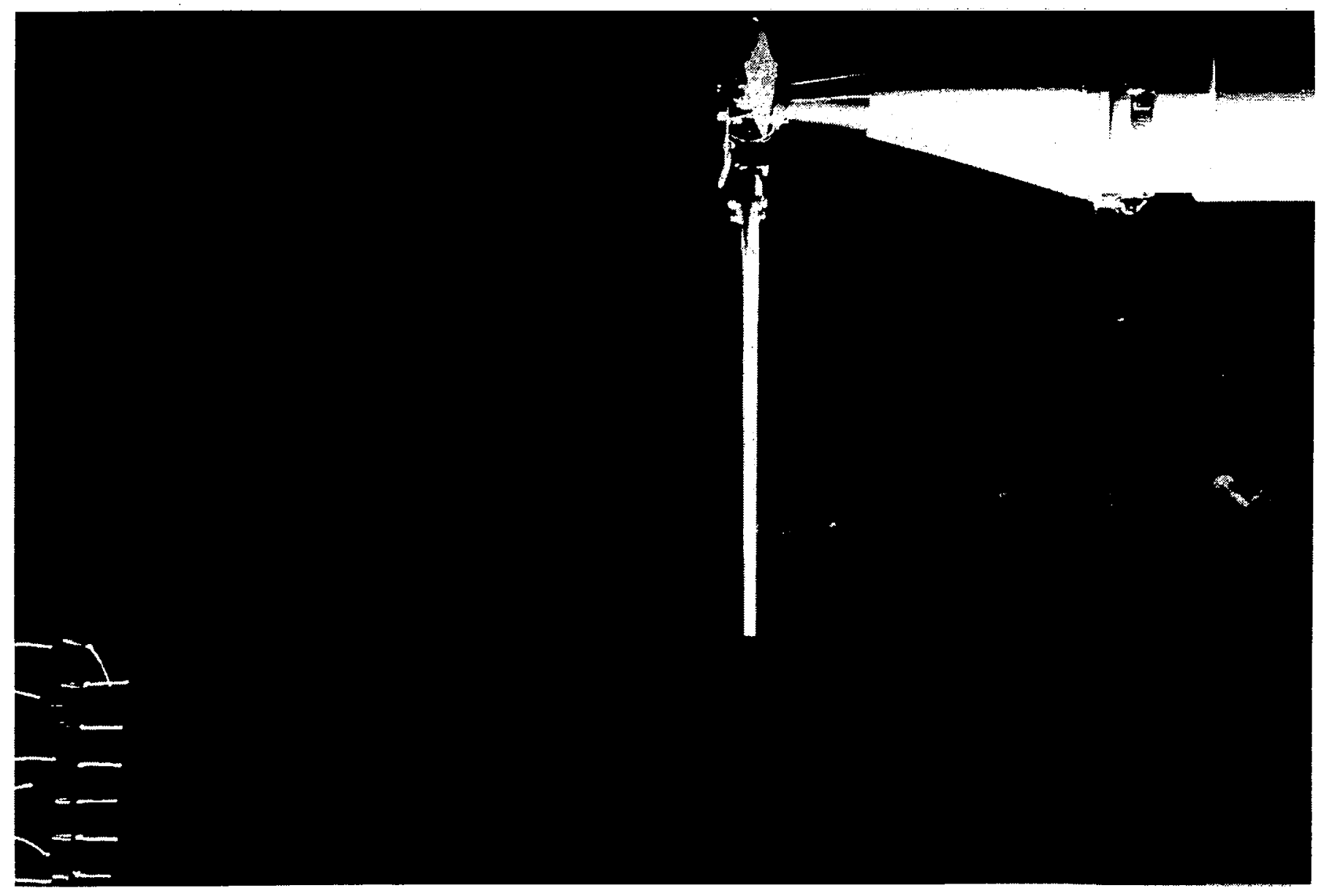

Figure 12 - Flow Visualization of Blade Vortex Interaction for Lifting Rotor 


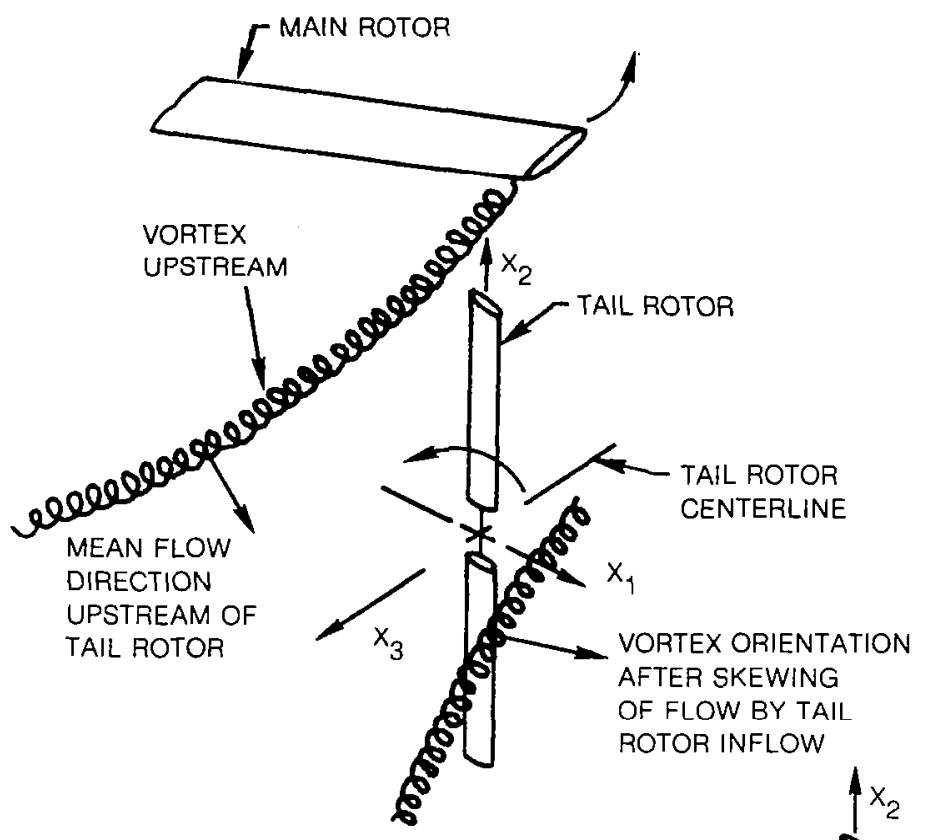

a) MAIN ROTOR VORTEX TRAJECTORY RELATIVE TO TAIL ROTOR AFTER APPLYING GALILEIAN TRANSFORMATION

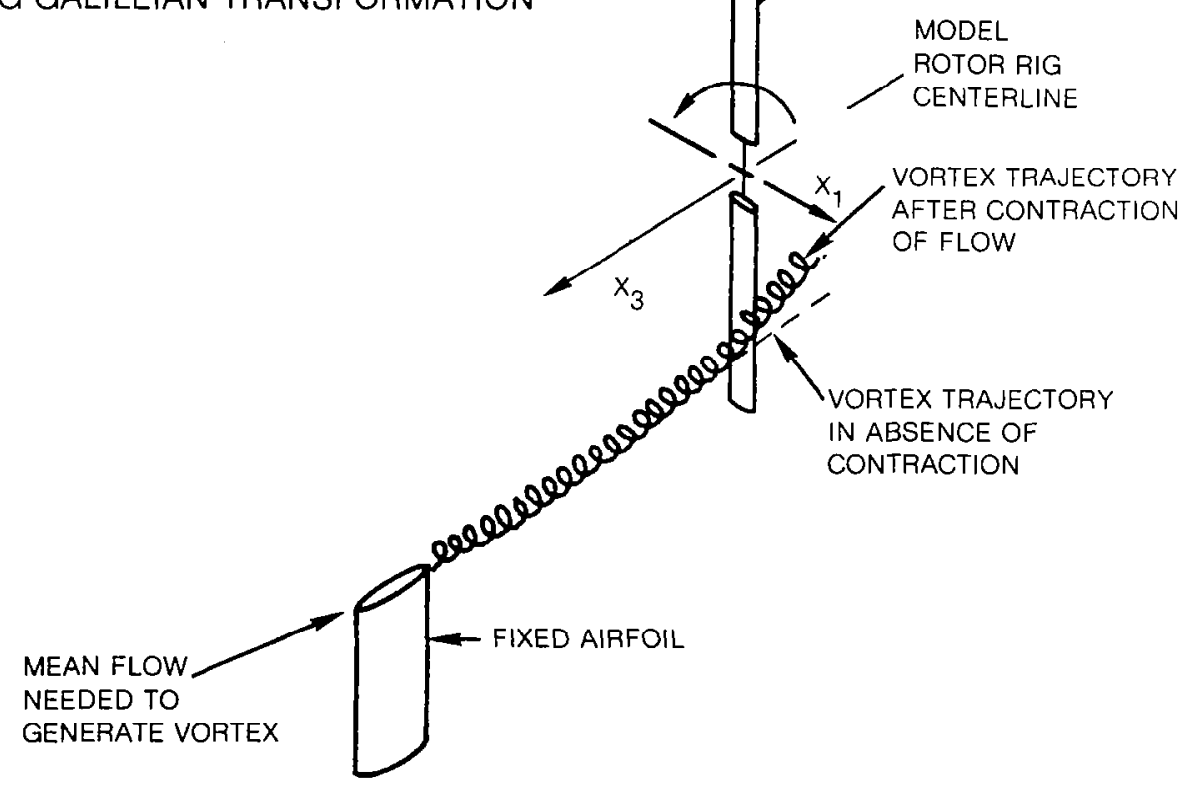

b) VORTEX TRAJECTORY IN OPEN JET WIND TUNNEL

WITH ROTOR

Figure 13 - Equivalence of Tail Rotor Vortex Interaction Process for Full Scale Helicopter and Open Jet Acoustic Wind Tunnel Model Rotor Experiment 


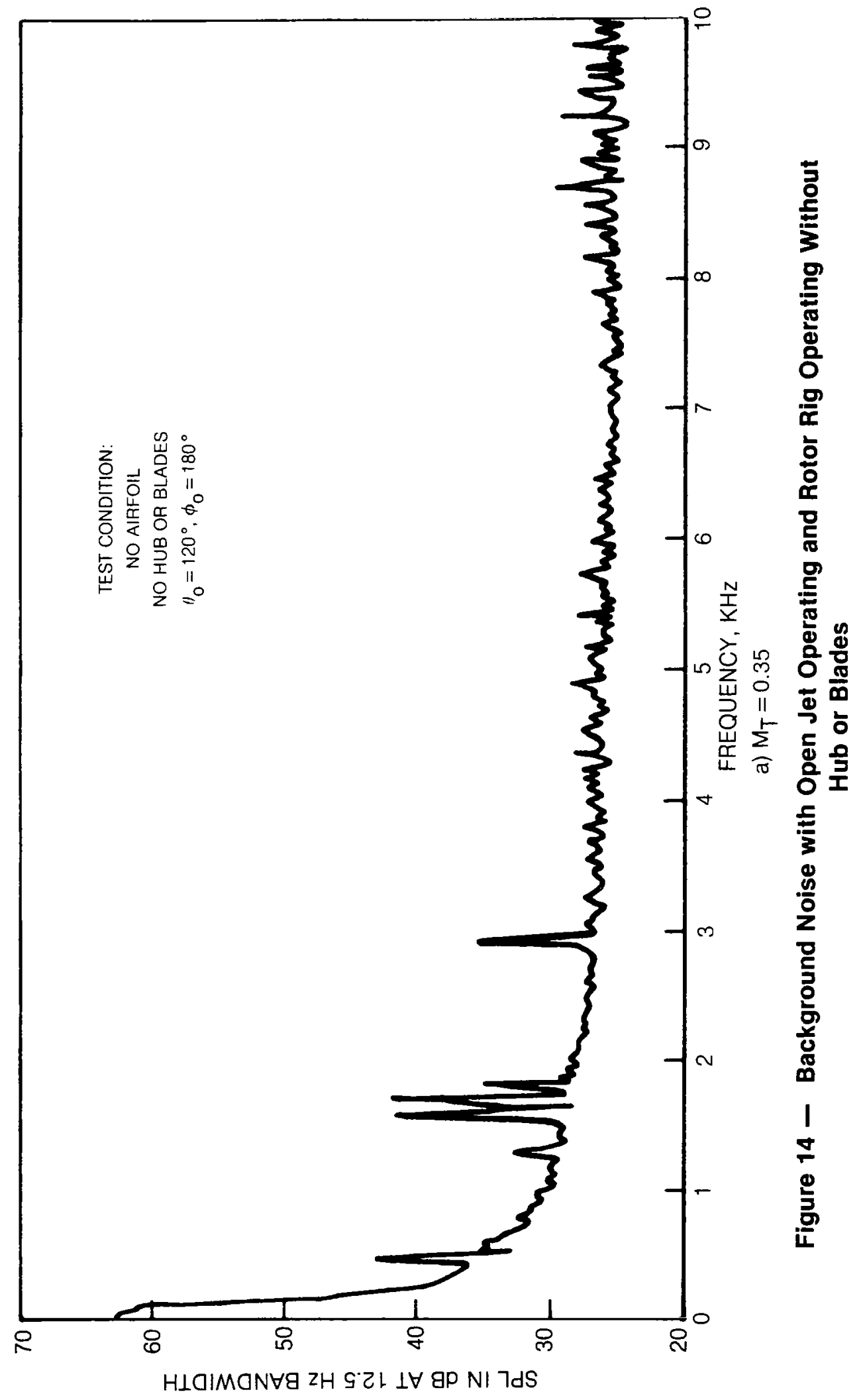




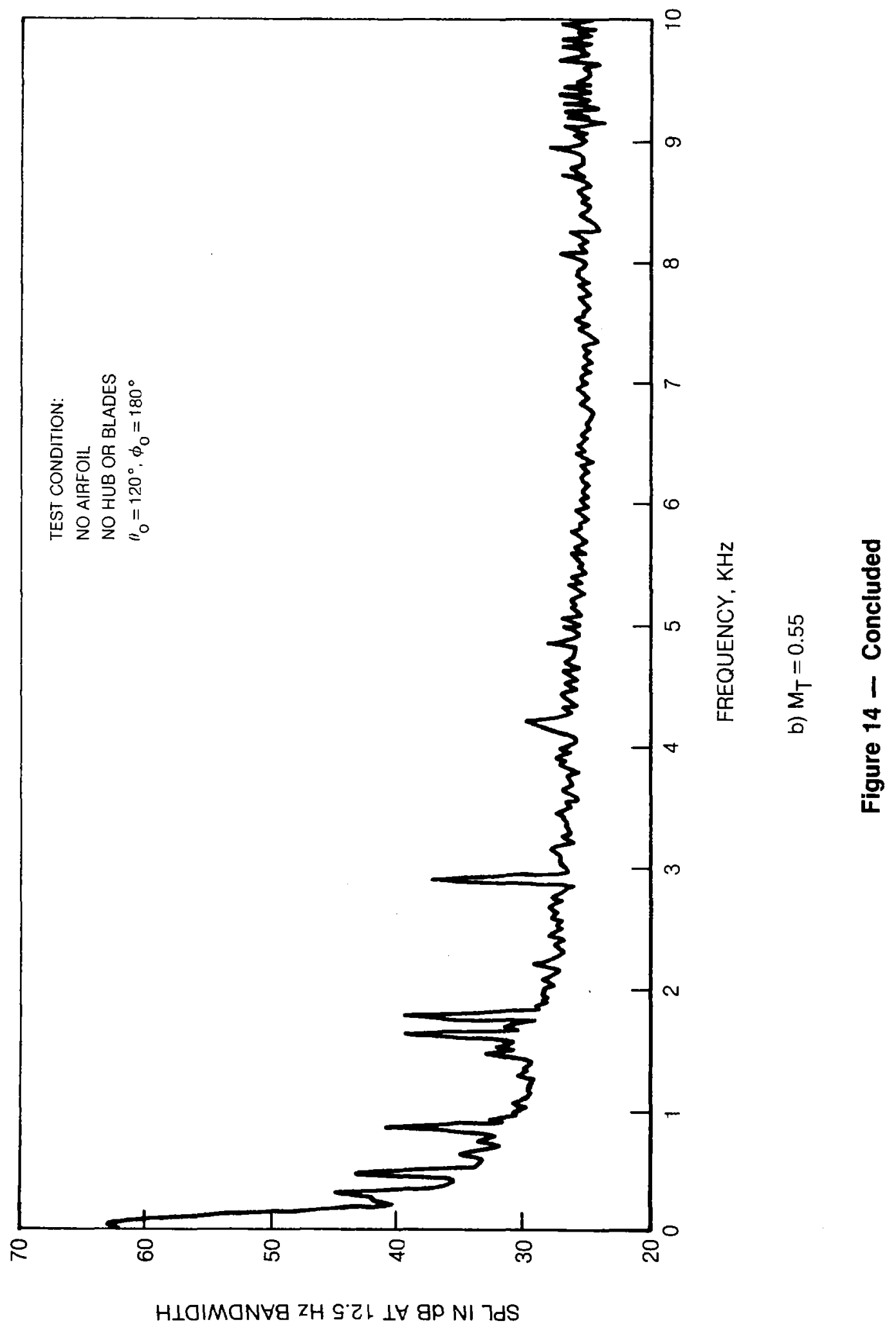




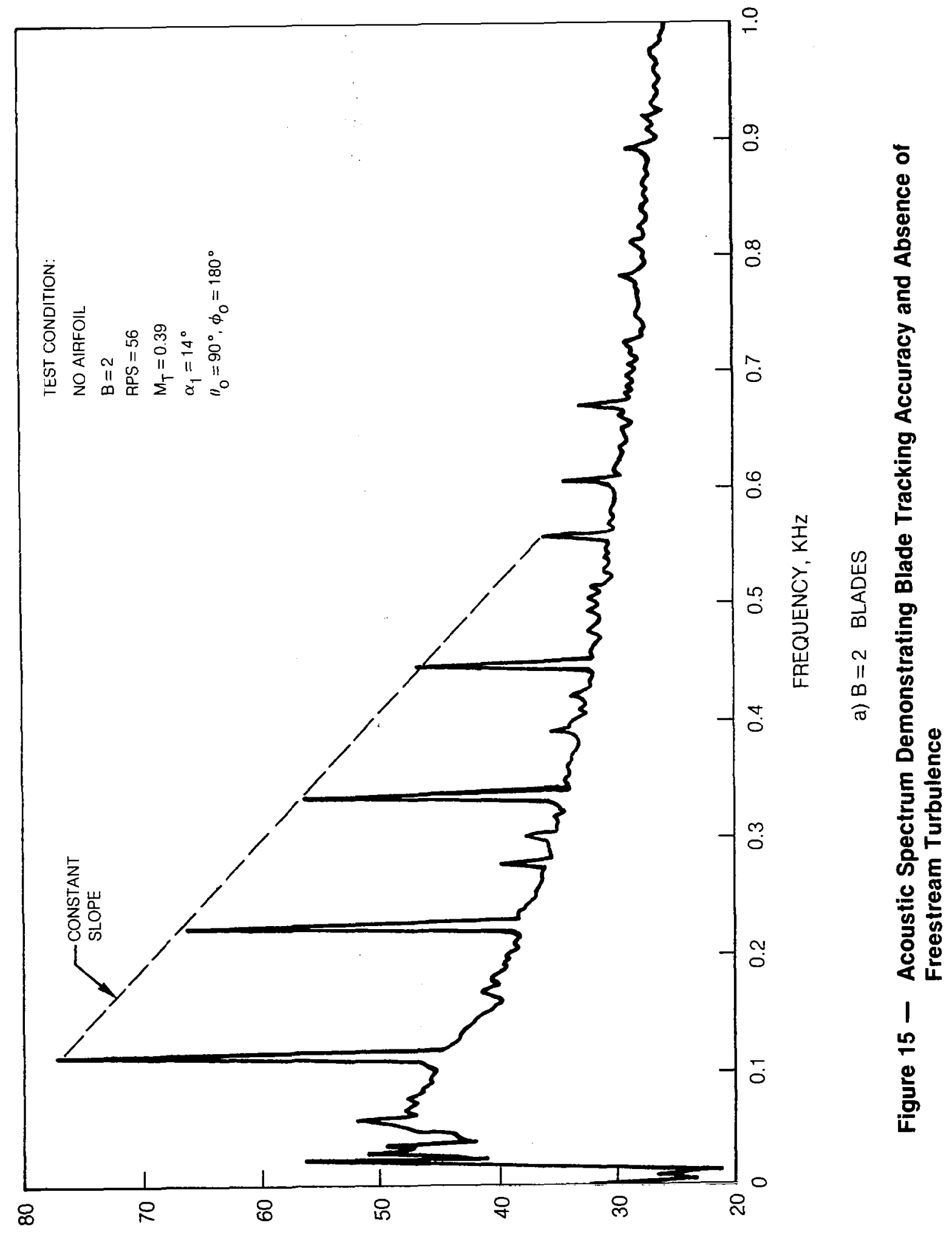

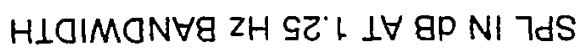




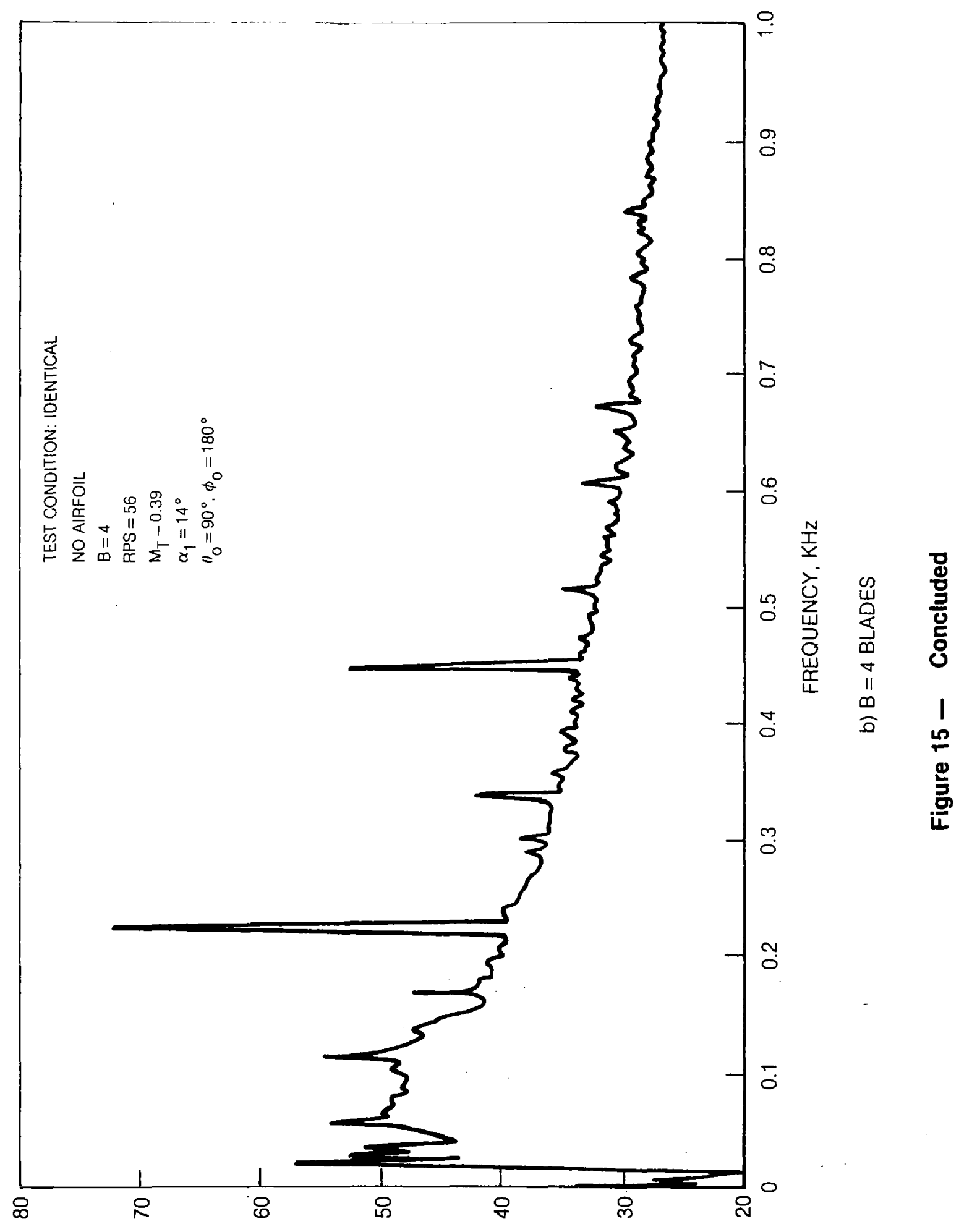

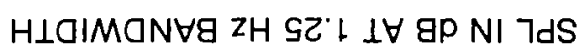




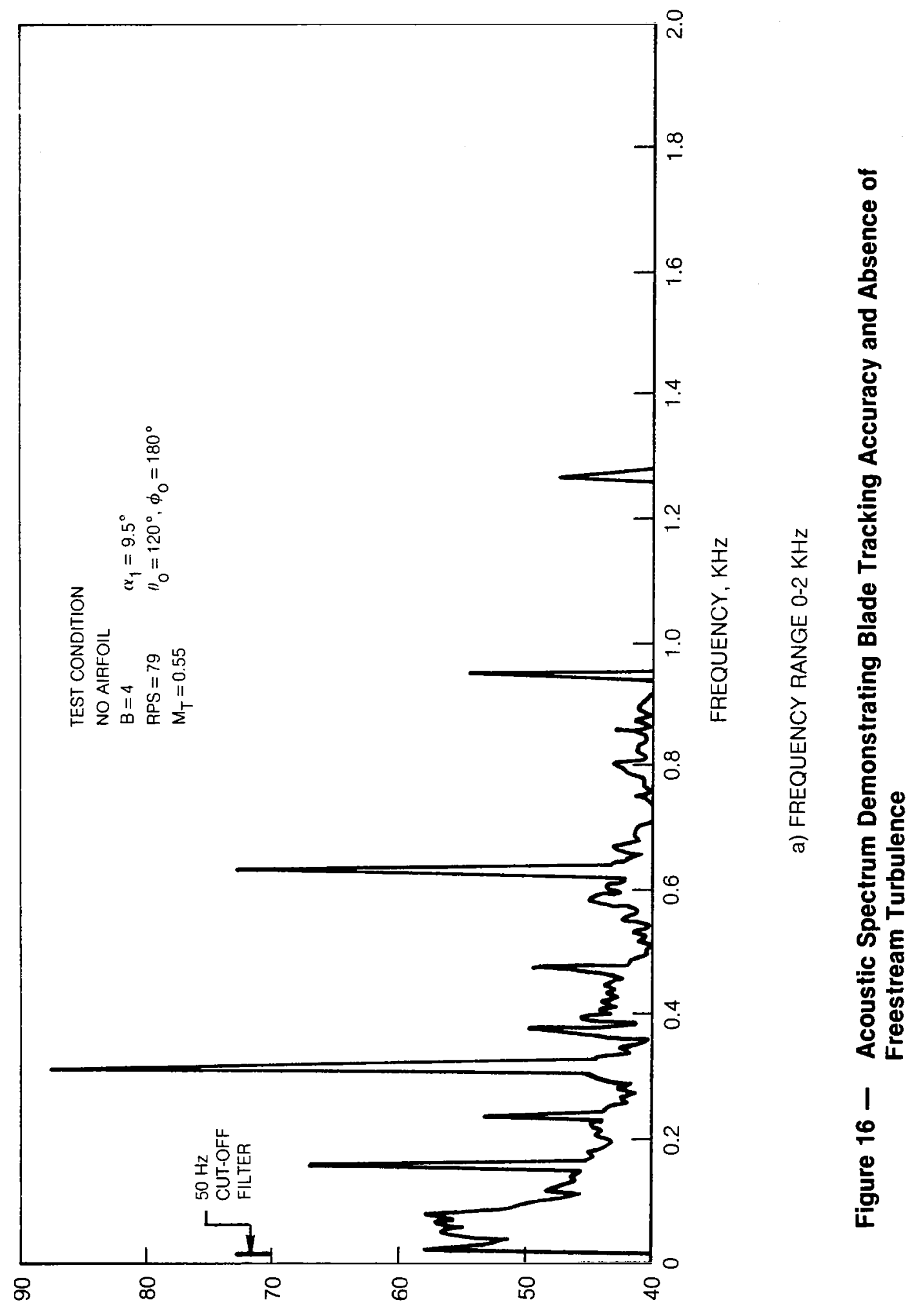

$H \perp O I M O N \forall B Z H G \cdot Z \perp \forall$ gP NI רdS 


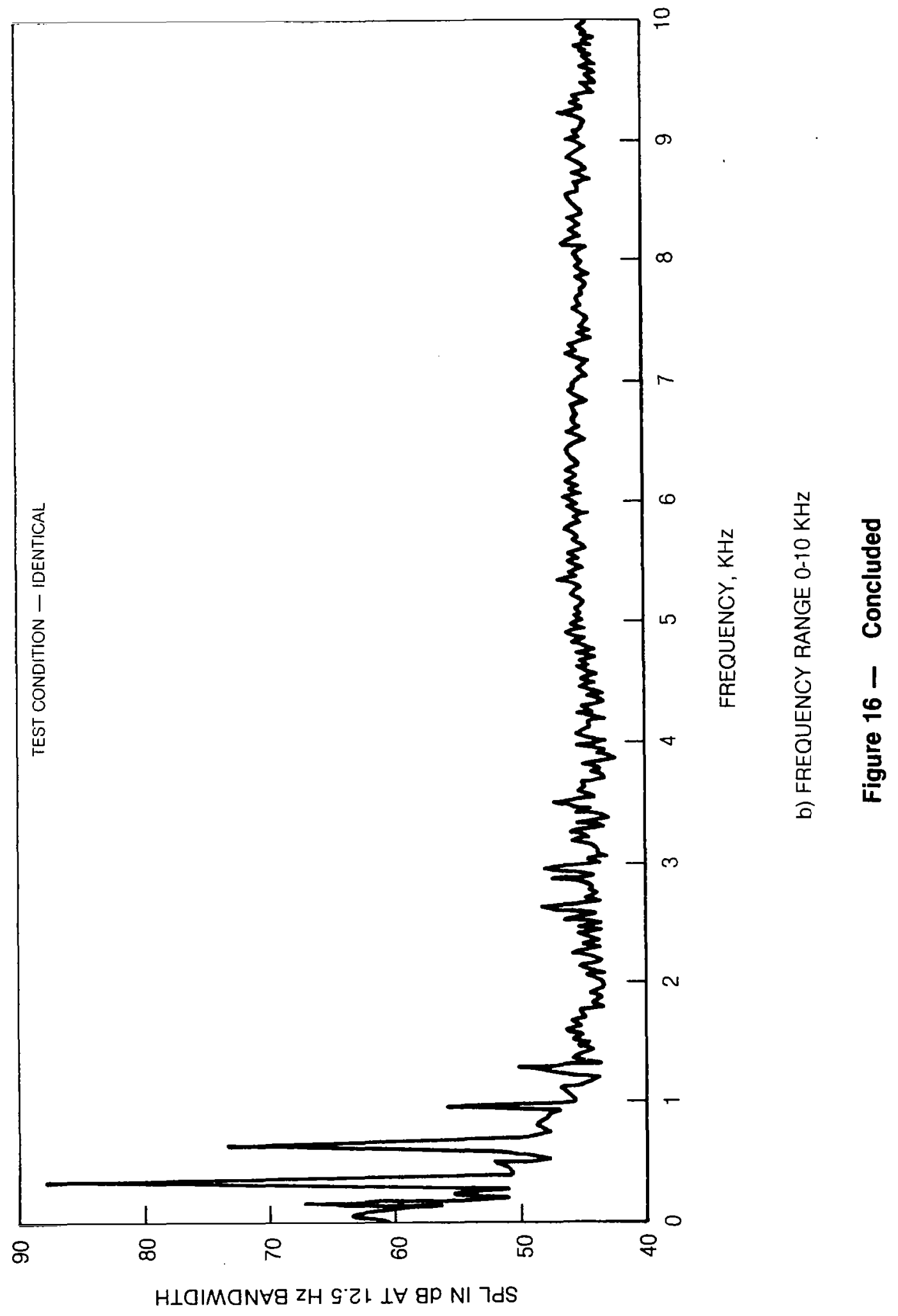




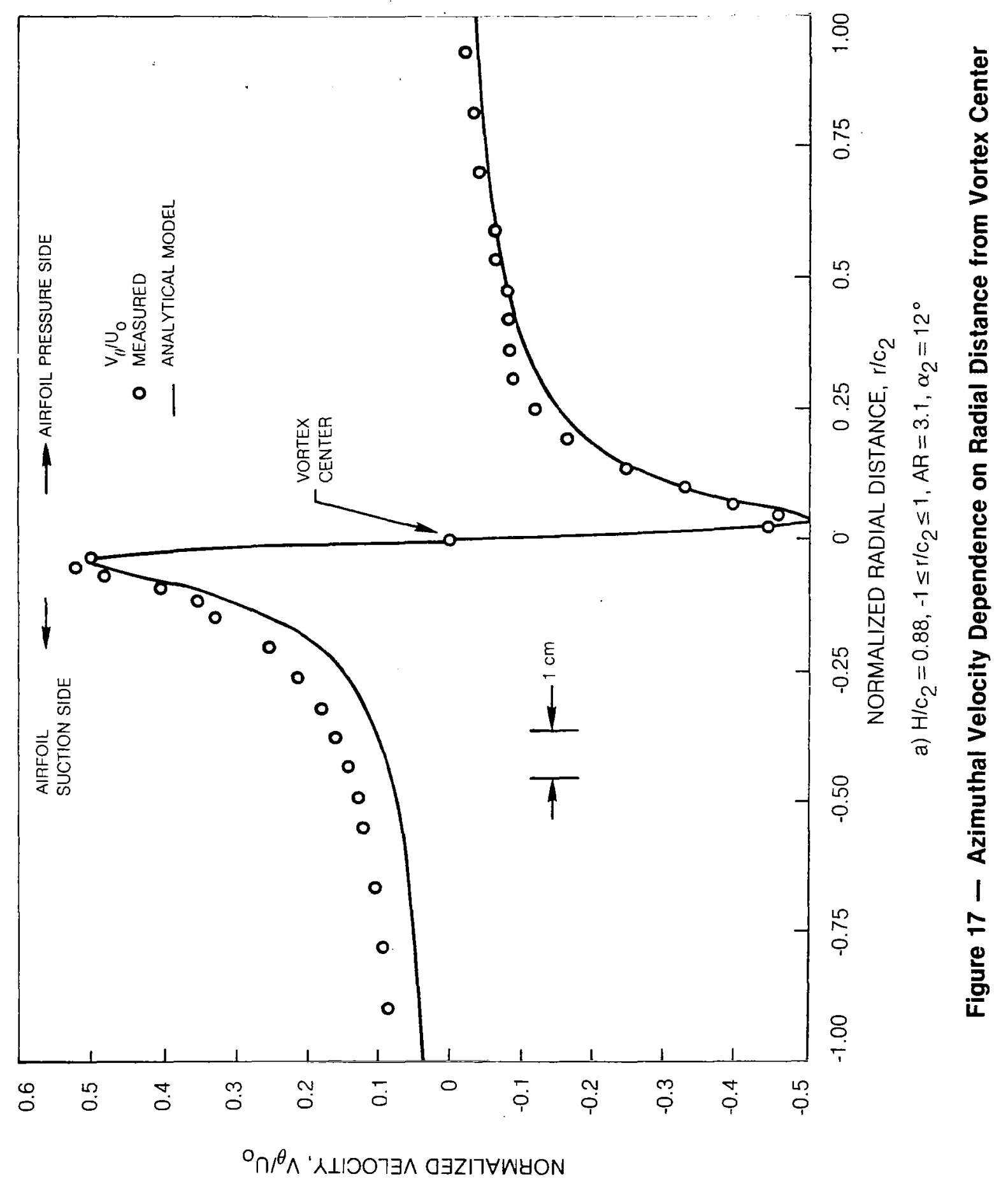




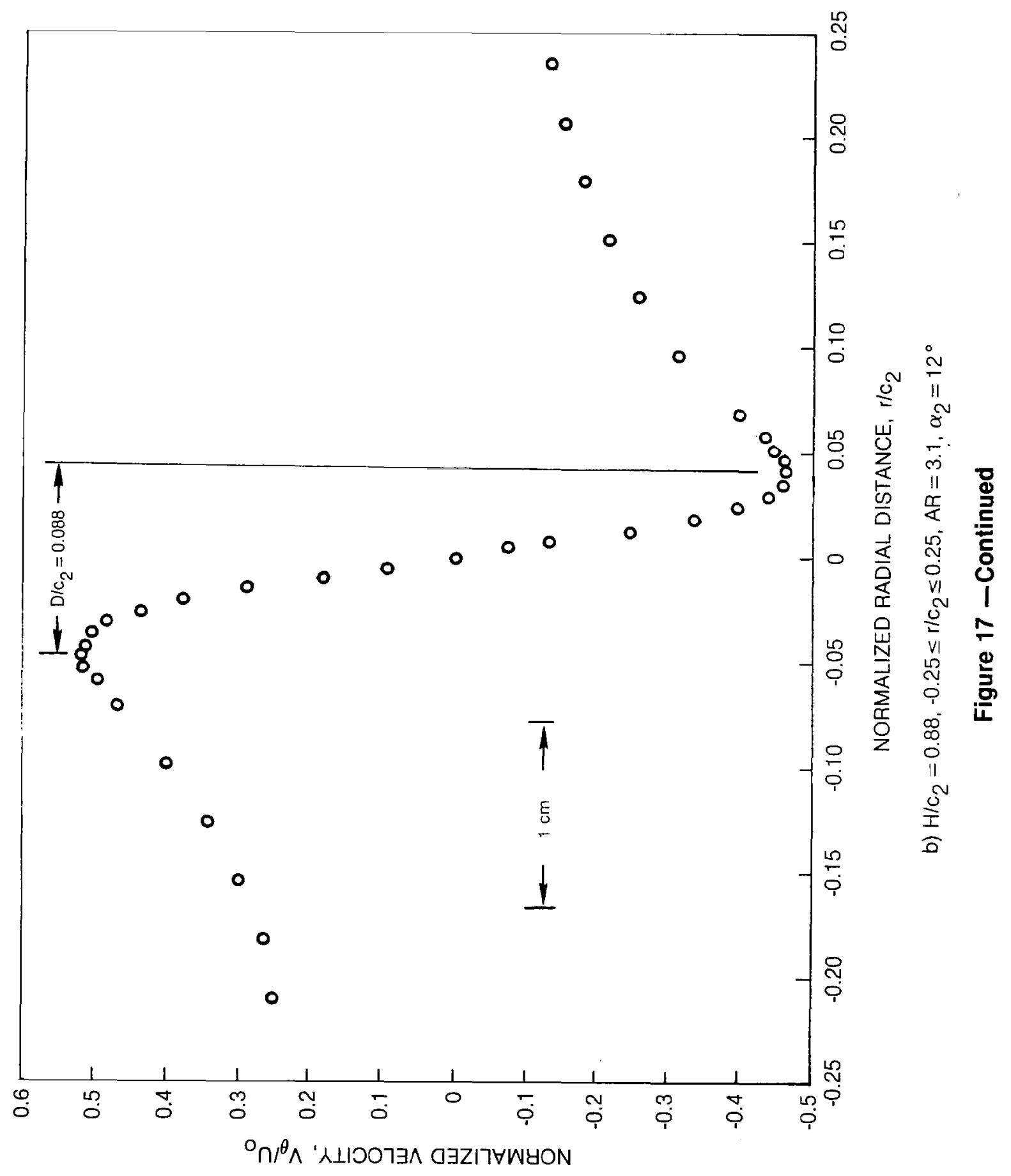




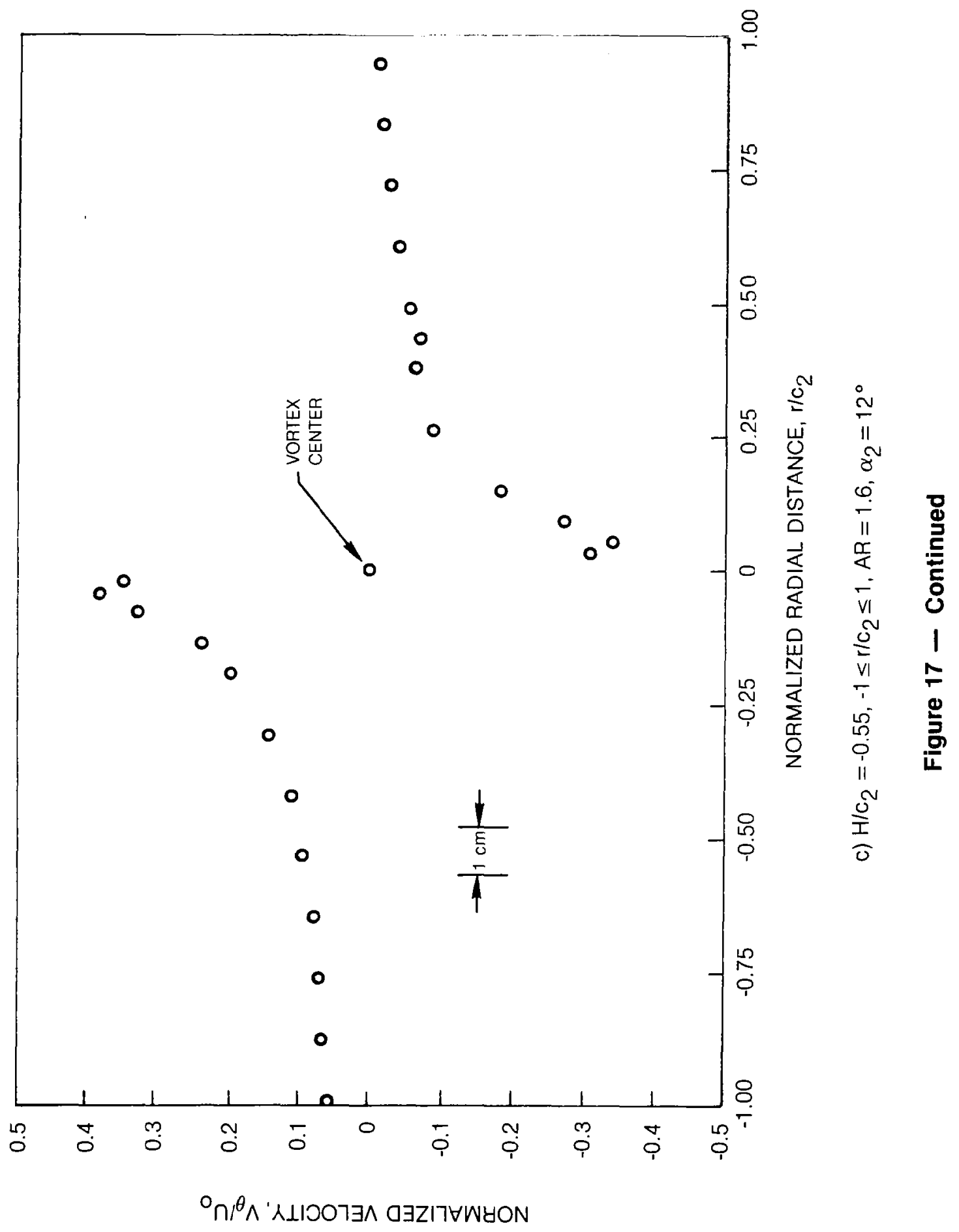




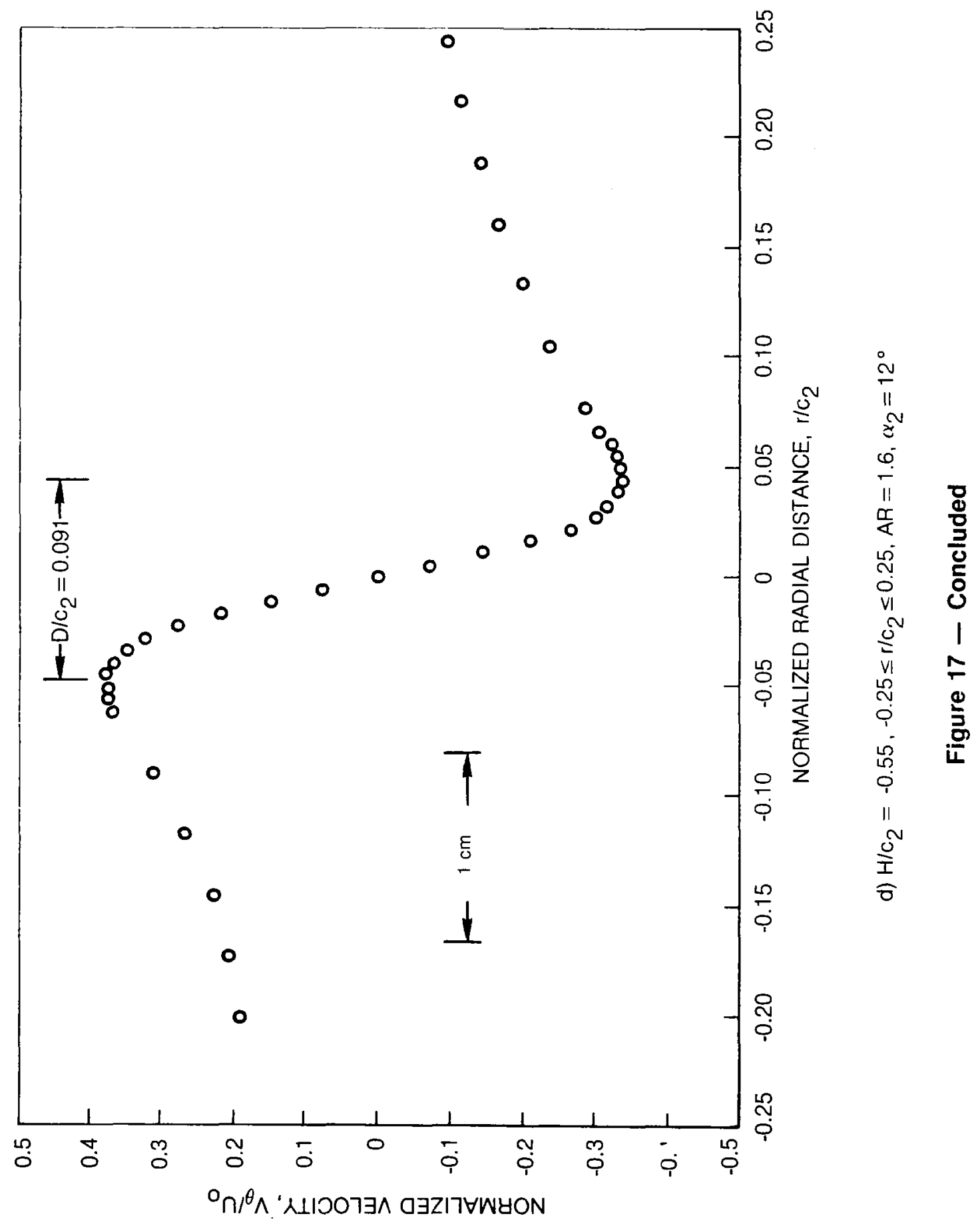


I III

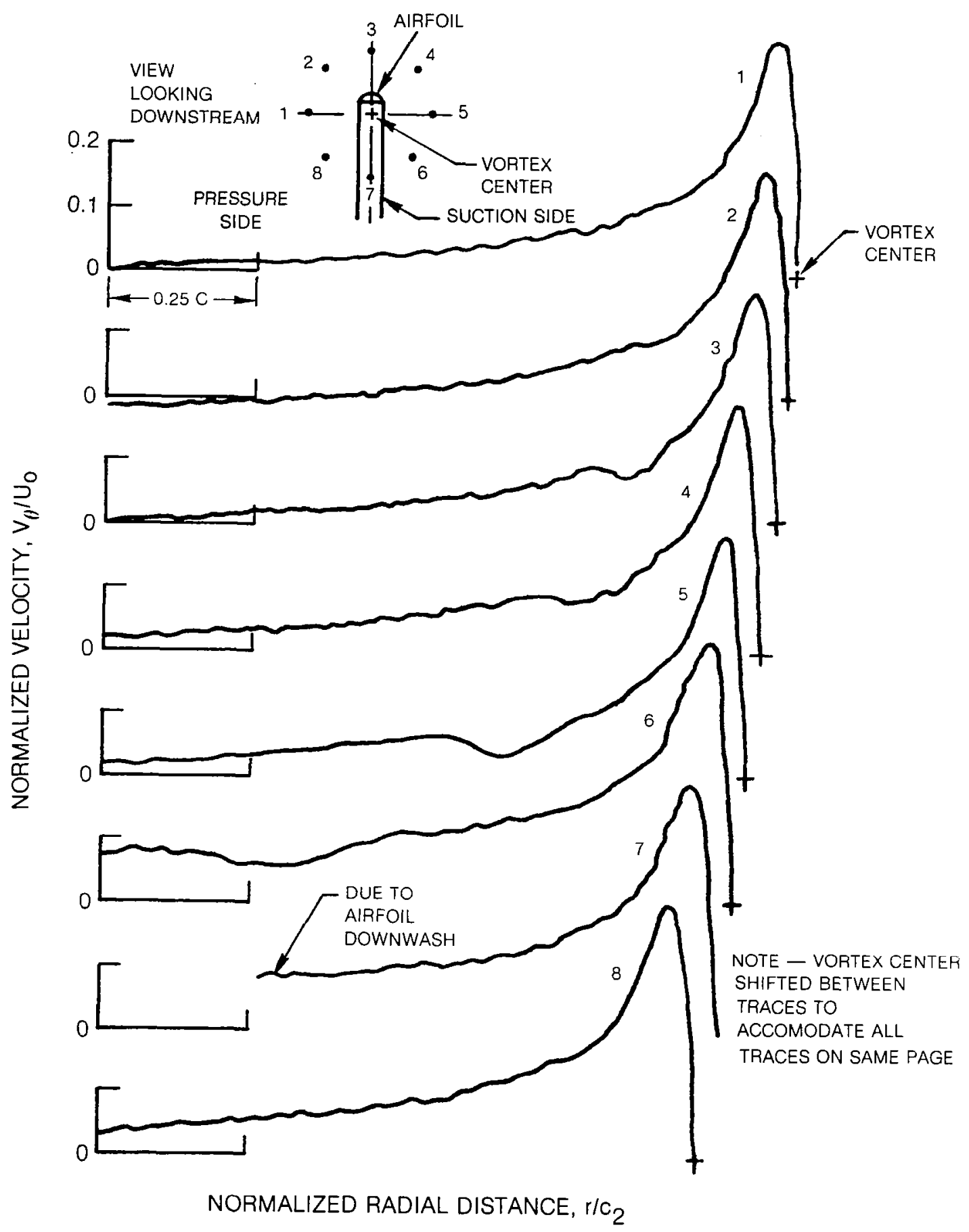

Figure 18 - Radial Traverses Showing Vortex Symmetry, $H / c_{2}=0, A R=2.22, \alpha_{2}=12^{\circ}$

84 


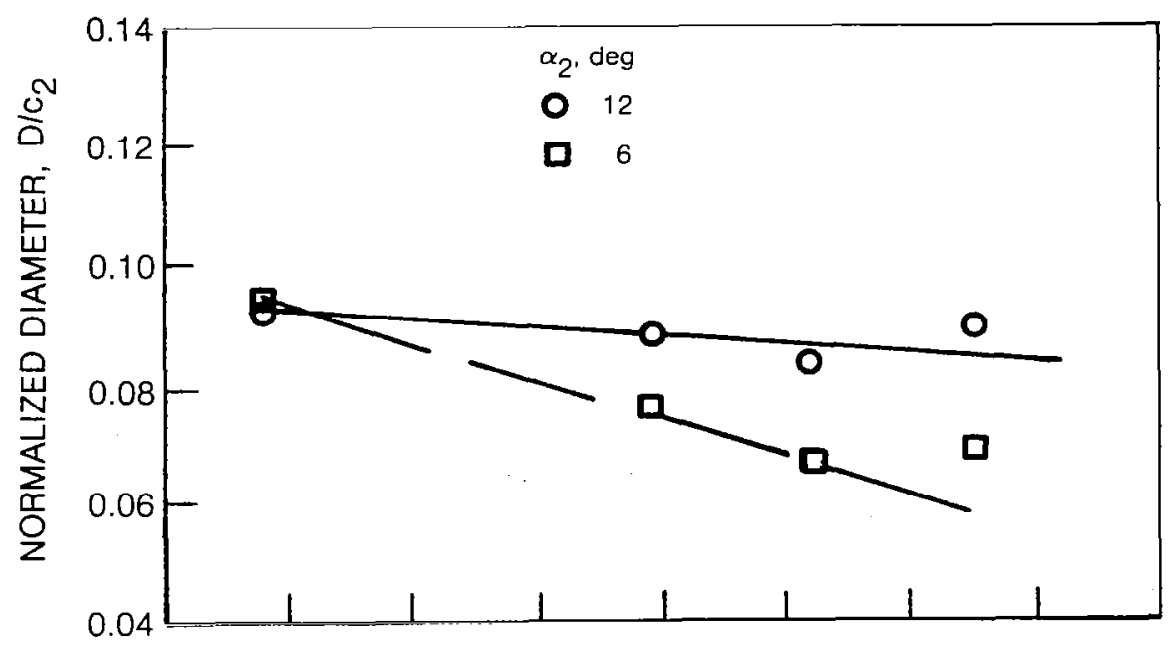

a) VORTEX CORE DIAMETER
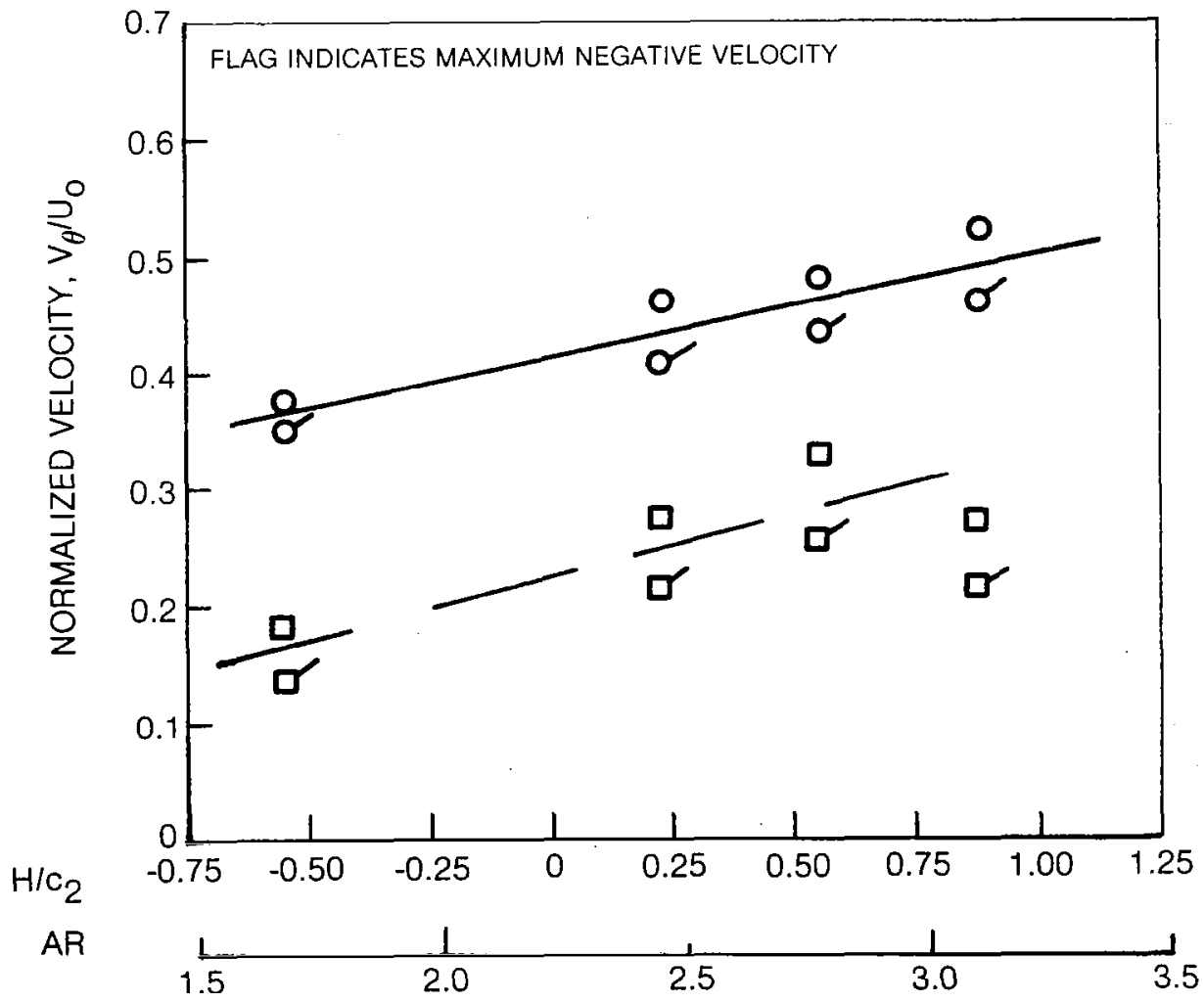

b) MAXIMUM AZIMUTHAL VELOCITY

Figure 19 - Vortex Core Size and Maximum Azimuthal Velocity. 


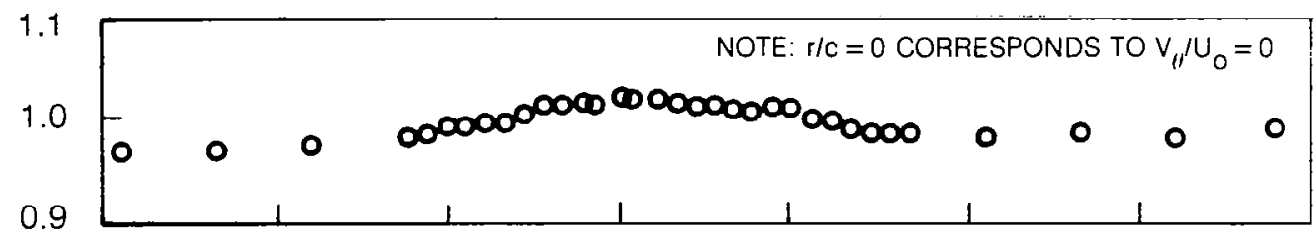

a) $H / c_{2}=-0.55$

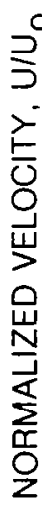

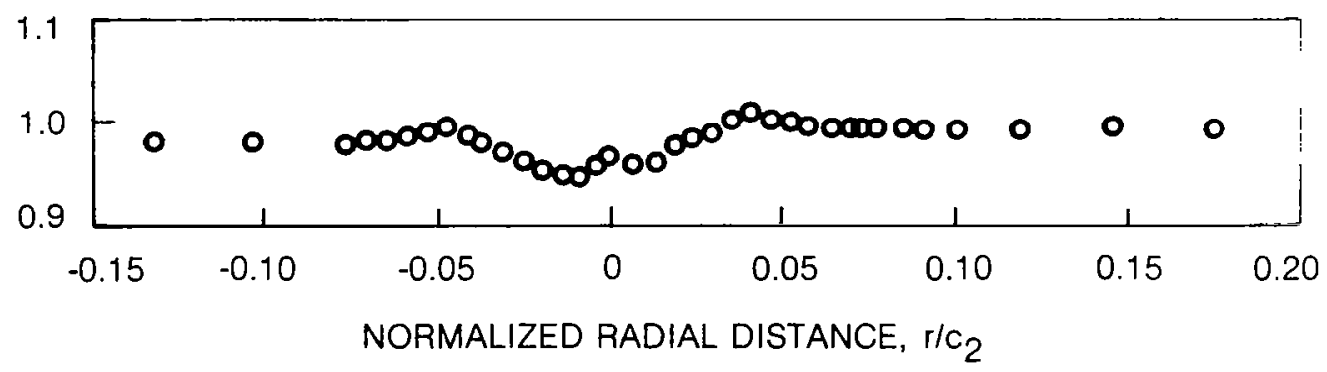

c) $\mathrm{H} / \mathrm{c}_{2}=0.55$

Figure 20 - Axial Velocity Dependence on Radial Distance from Vortex Center 

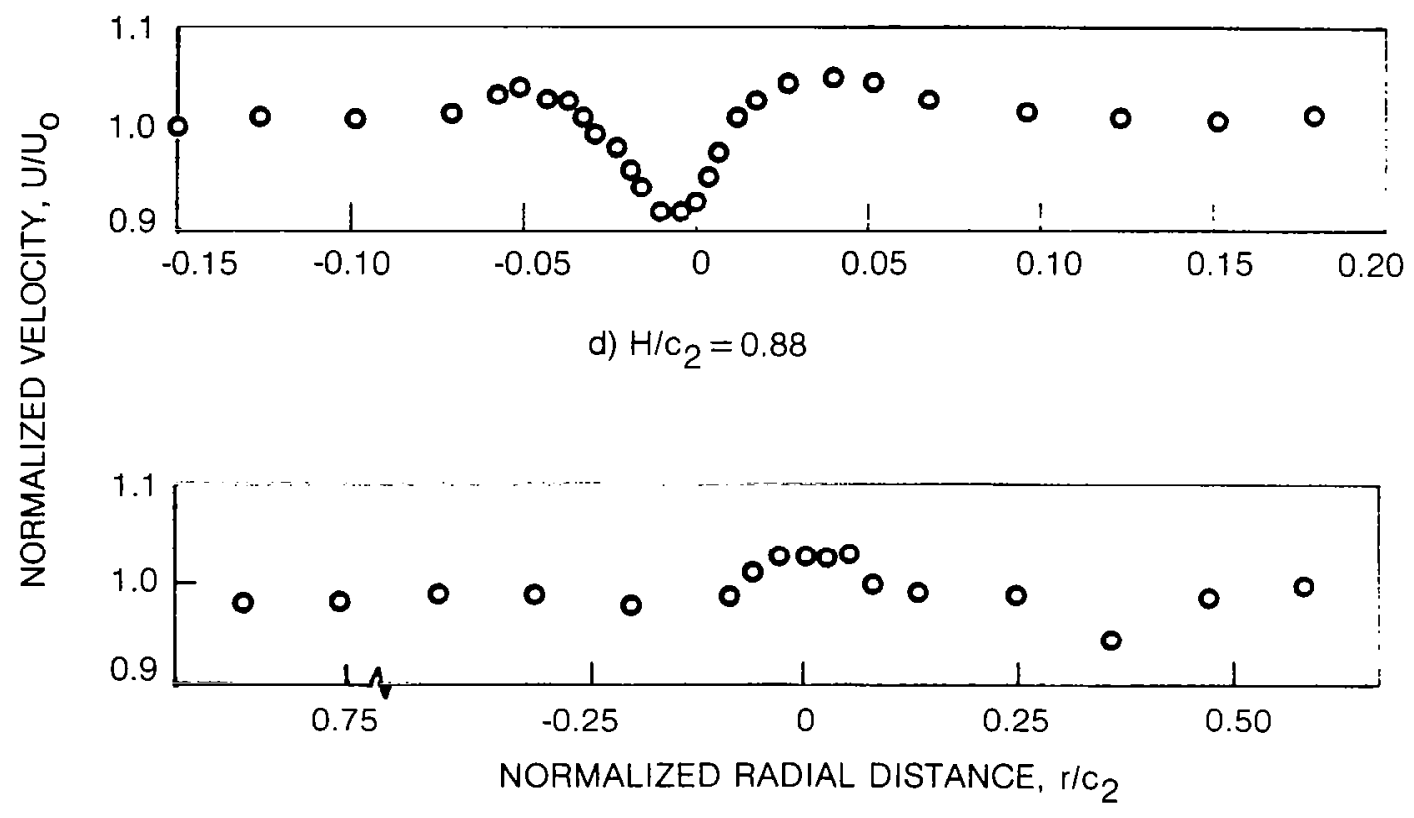

e) $\mathrm{H} / \mathrm{C}_{2}=-0.55$

Figure 20 - Concluded 


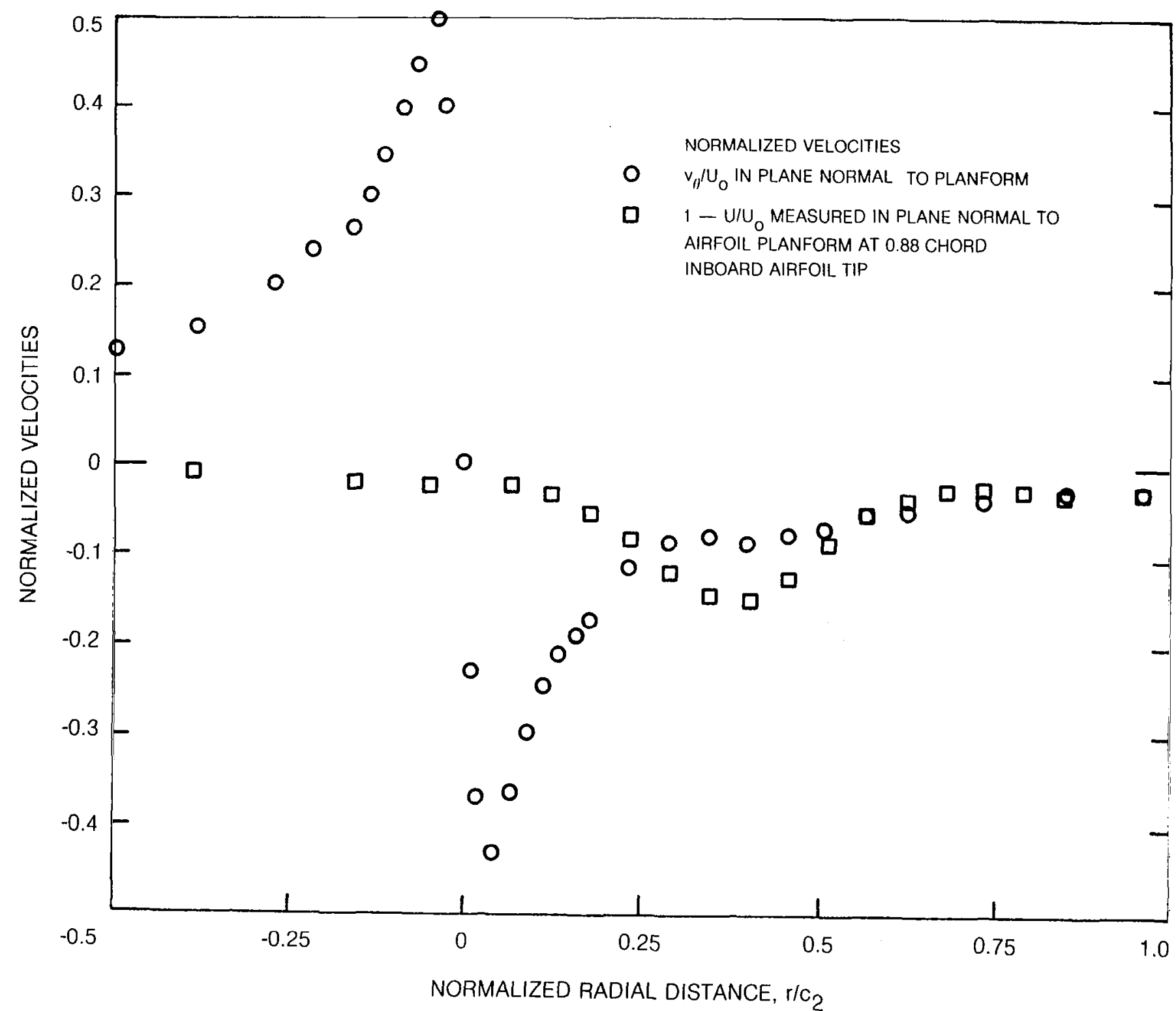

Figure 21 - Comparison of Vortex and Two Dimensional Wake Velocity Field, $\mathrm{H} / \mathrm{c}_{2}=\mathbf{0 . 5 5}$, $A R=2.77, \alpha_{2}=12^{\circ}$ 


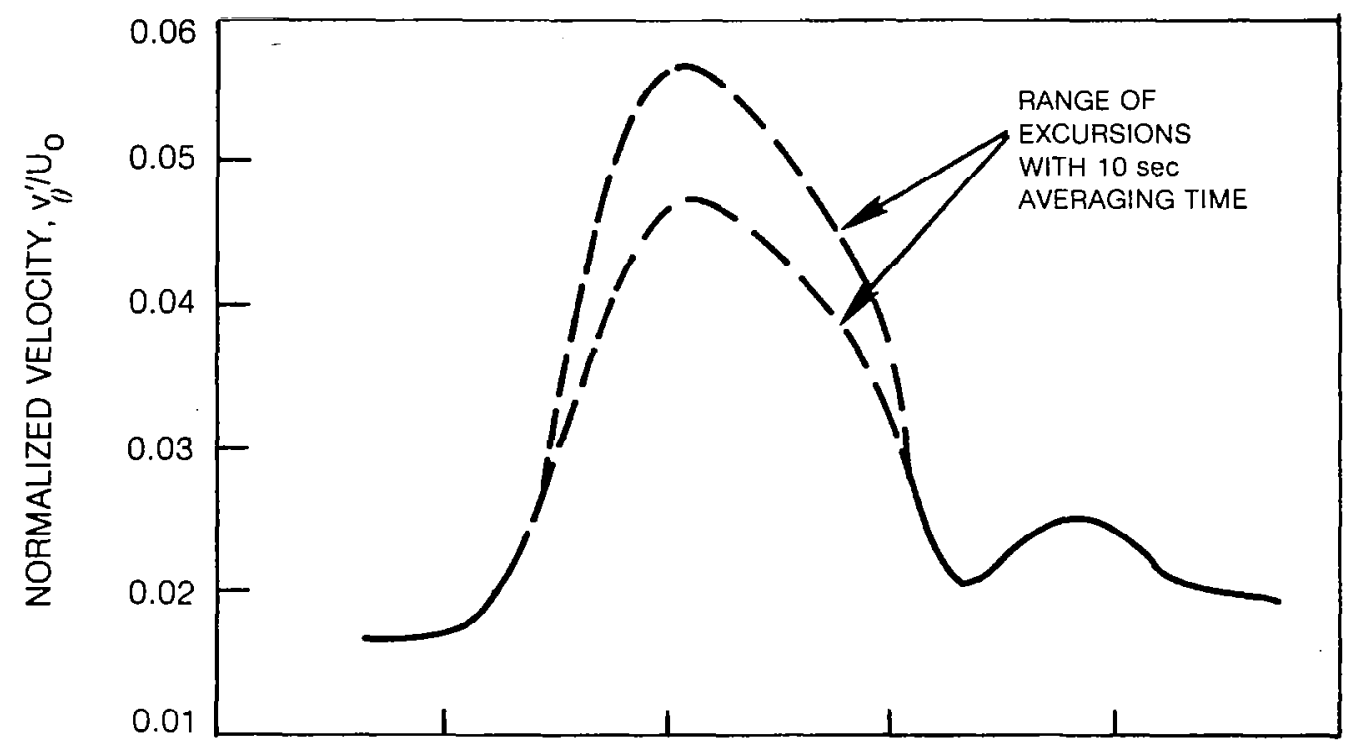

a) $v_{\theta}^{\prime}$ VARIATION WITH $r$

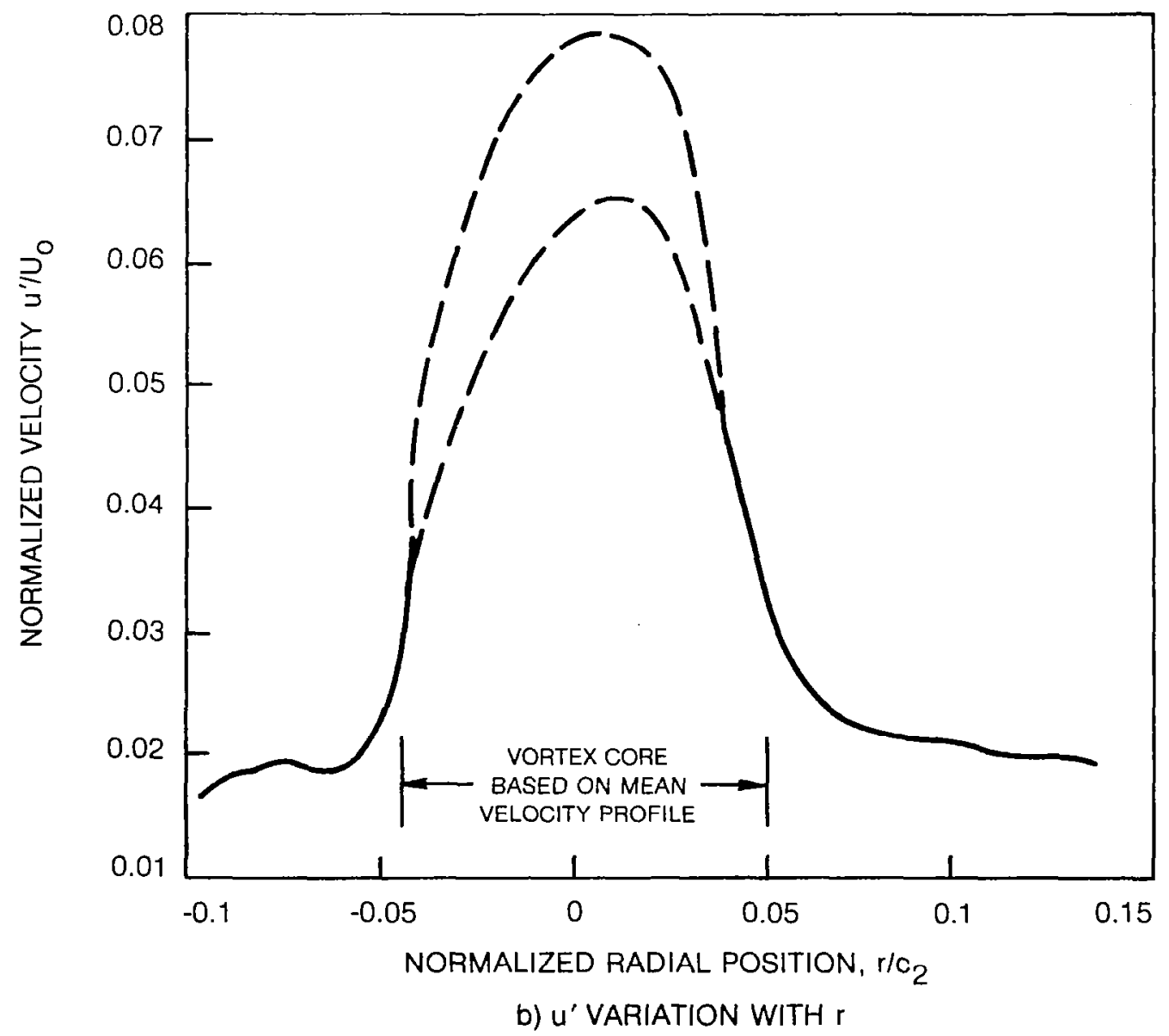

Figure 22 - Turbulence Intensity Components $v^{\prime}{ }_{\theta}$ and $u{ }^{\prime}$ in Vortex Viscous Core 


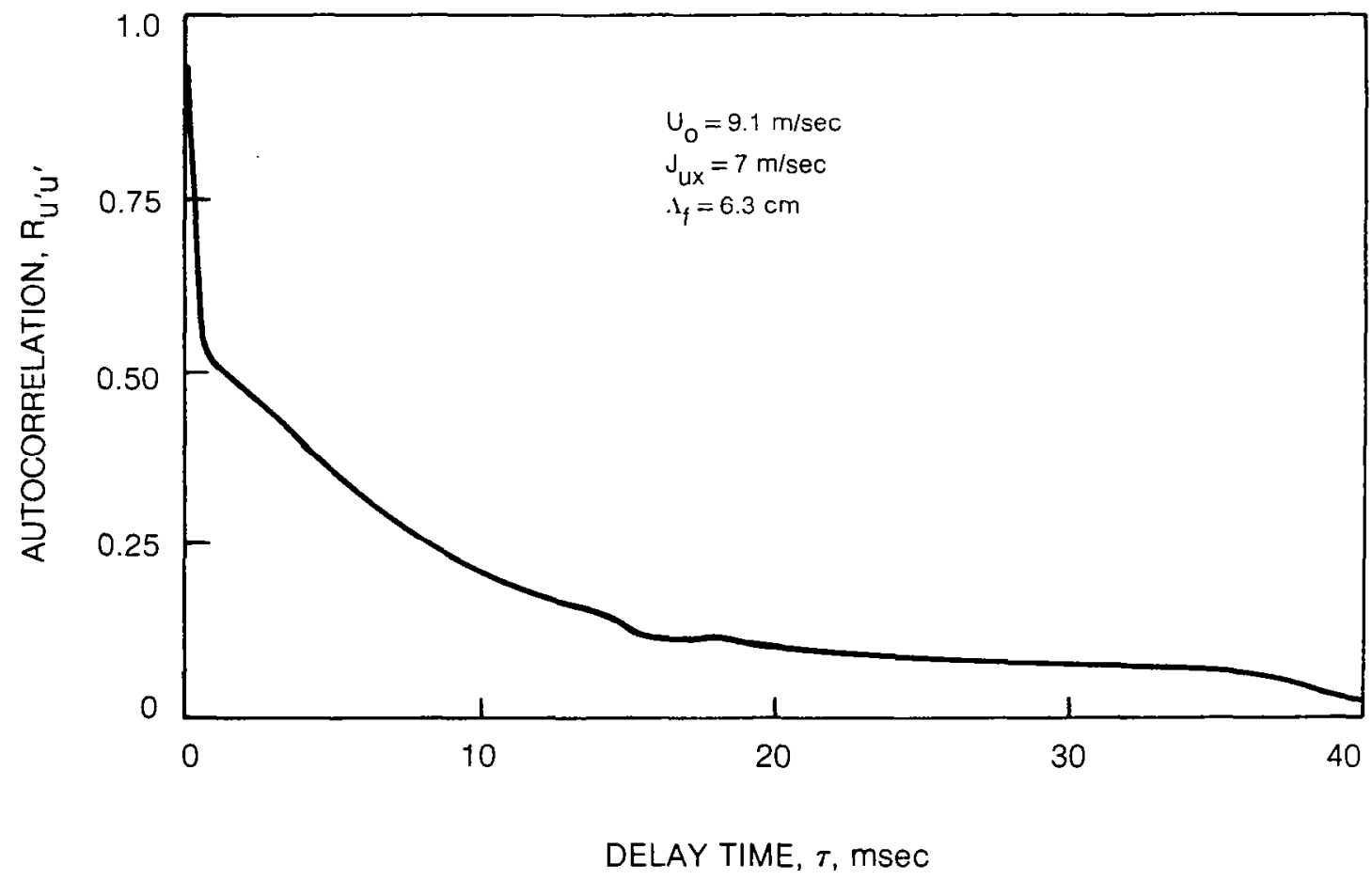

Figure 23 - Autocorrelation of Axial Turbulence Component, $u^{\prime}$ 


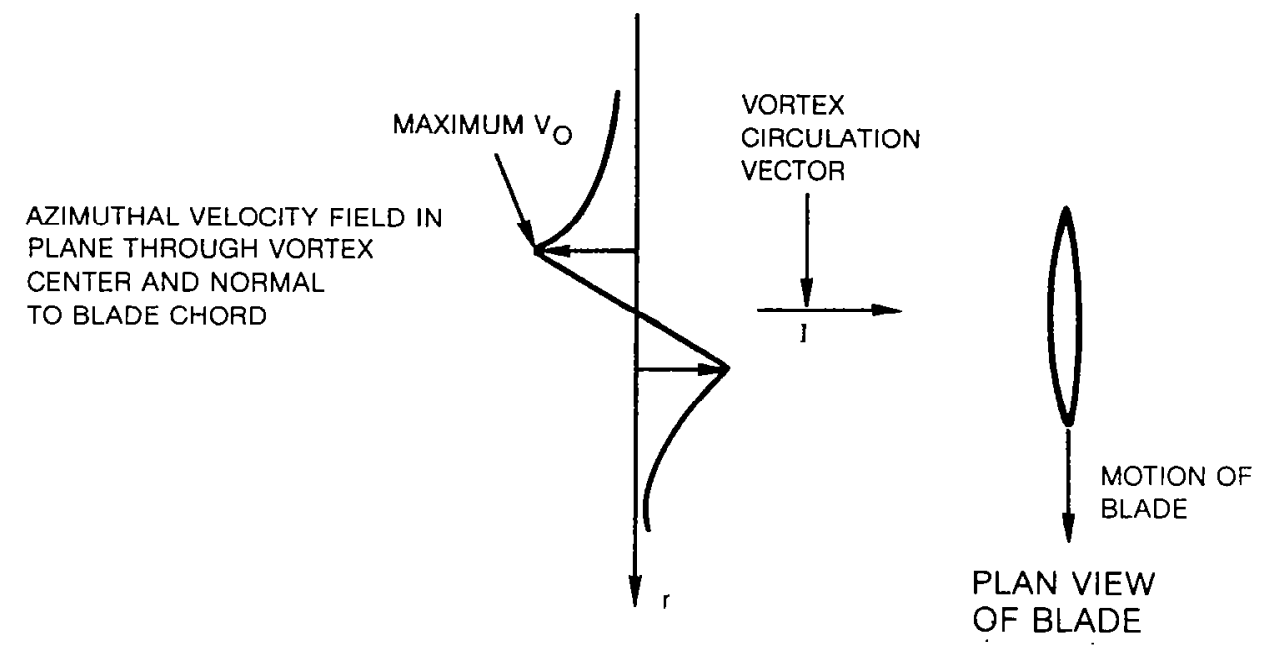

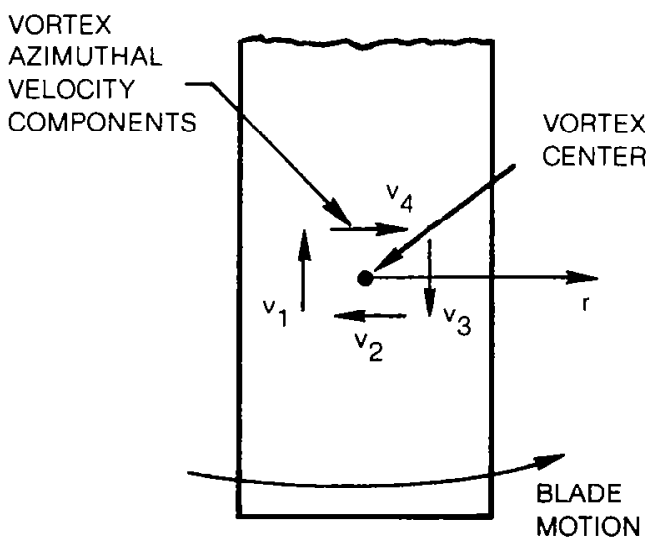

FRONT VIEW

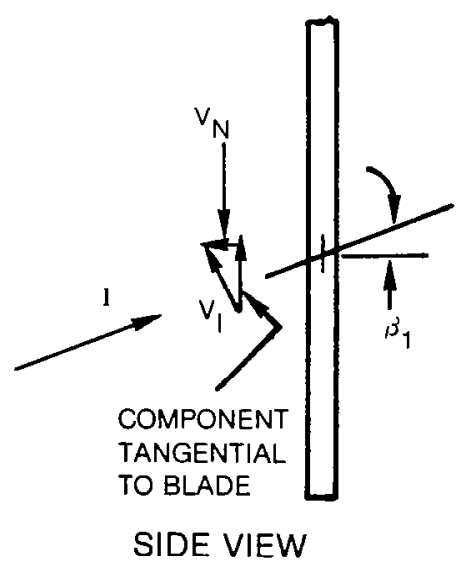

Figure 24 - Schematic of Vortex Velocity Components Responsible for Noise Generation 


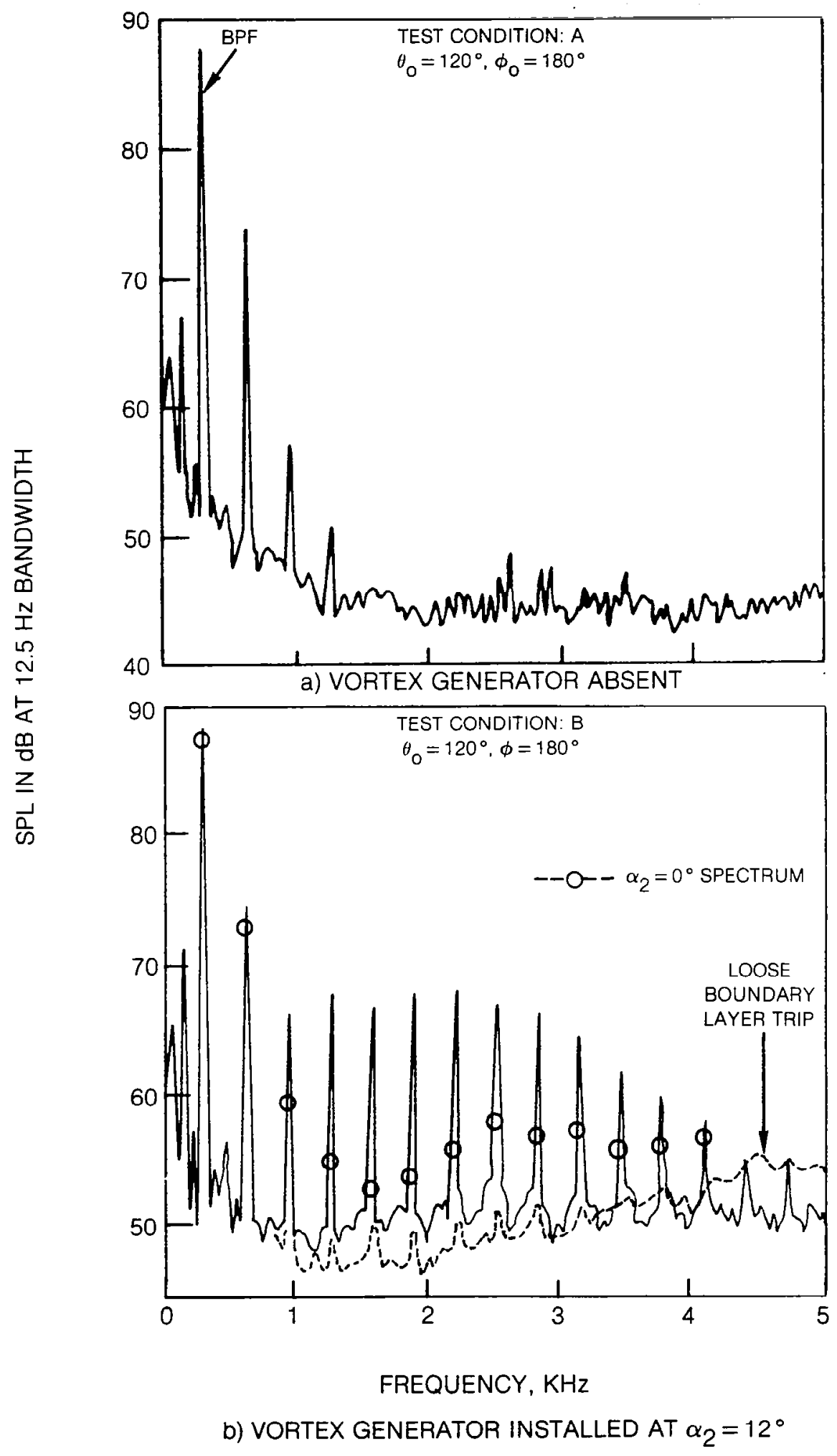

Figure 25 - Effect of Vortex Generator on Acoustic Spectrum 


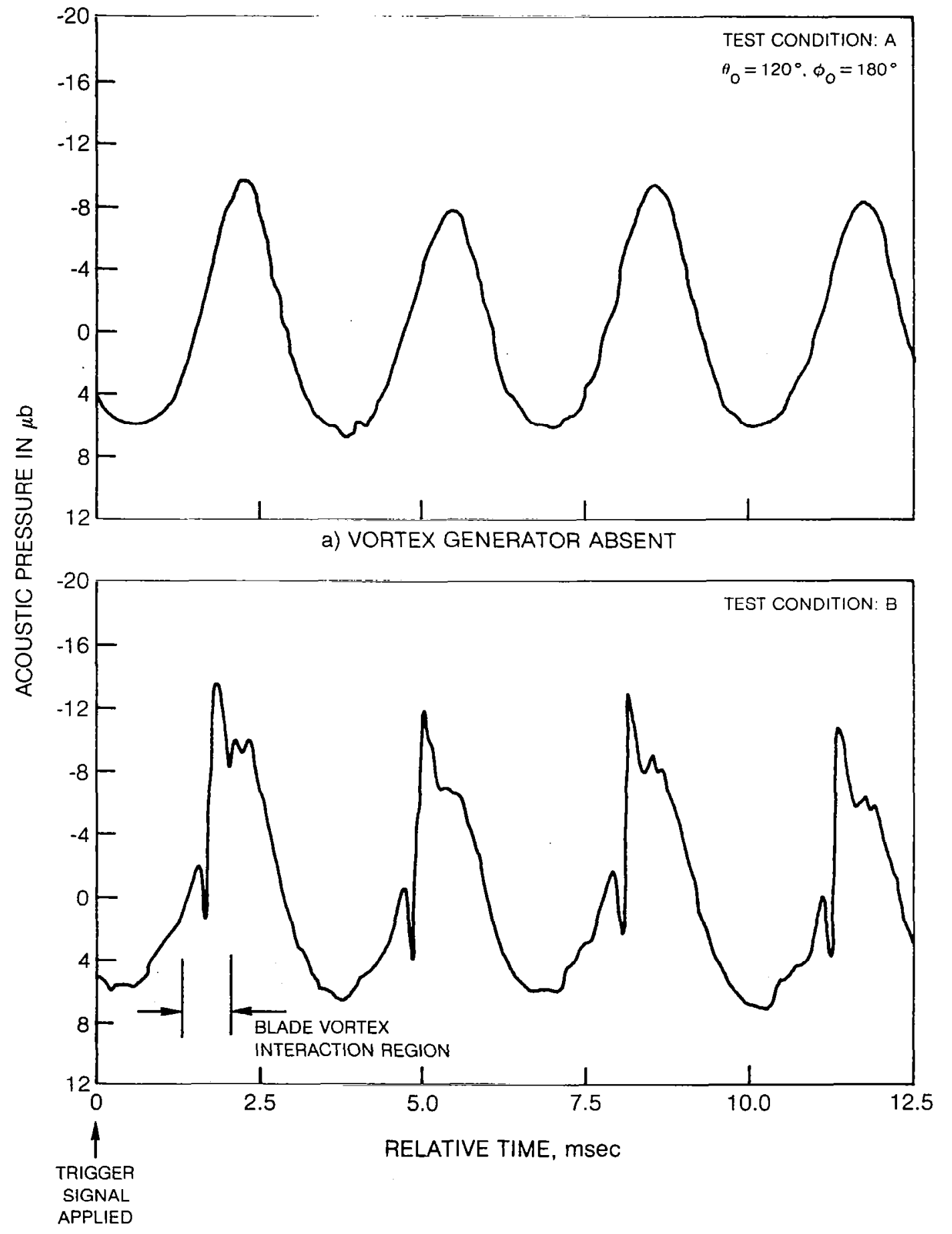

b) VORTEX GENERATOR INSTALLED AT $\alpha_{2}=12^{\circ}$

Figure 26 - Effect of Vortex Generator on Acoustic Signature 


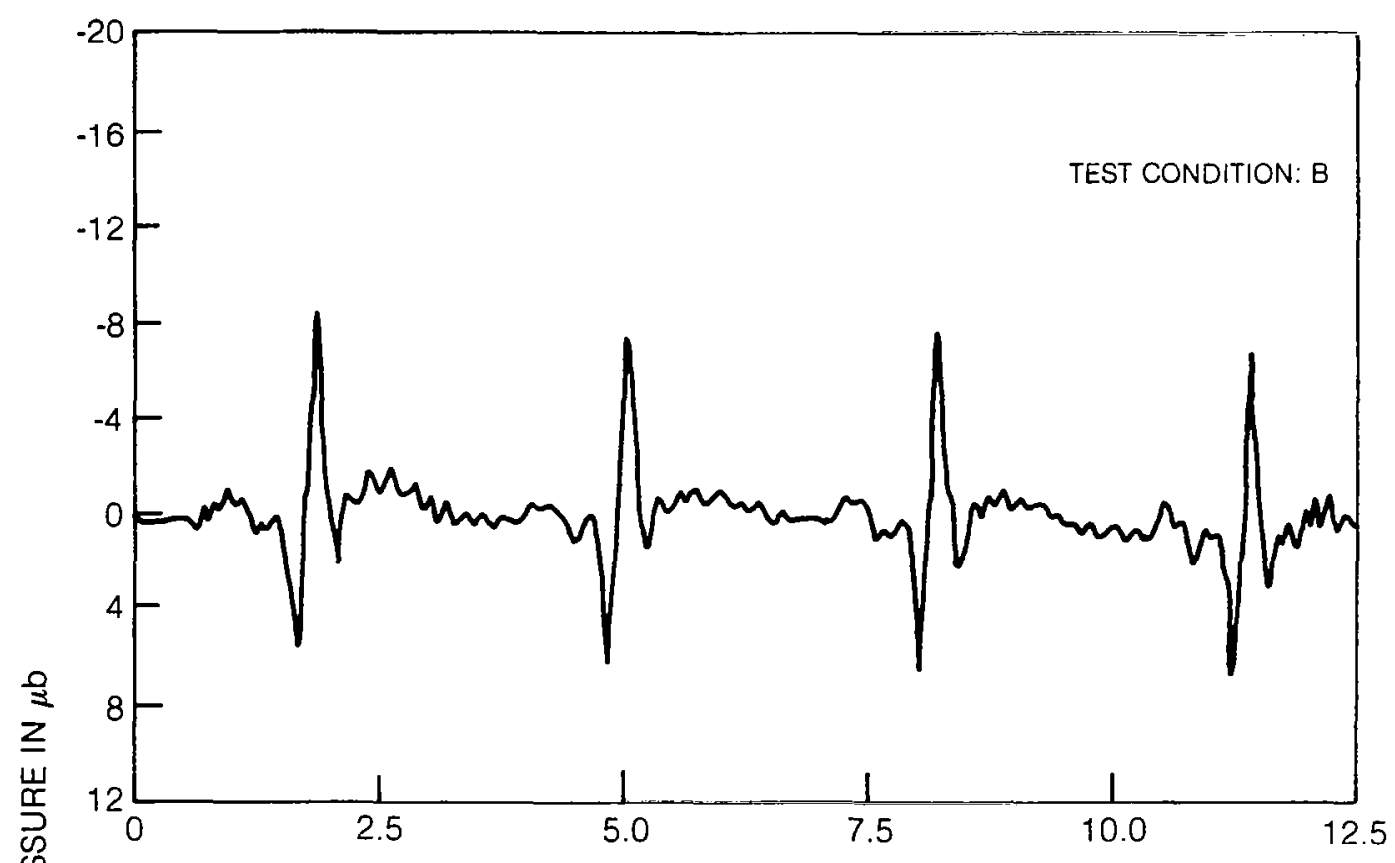

c) ISOLATED BLADE VORTEX INTERACTION TIME HISTORY

$\frac{0}{\frac{0}{6}}$

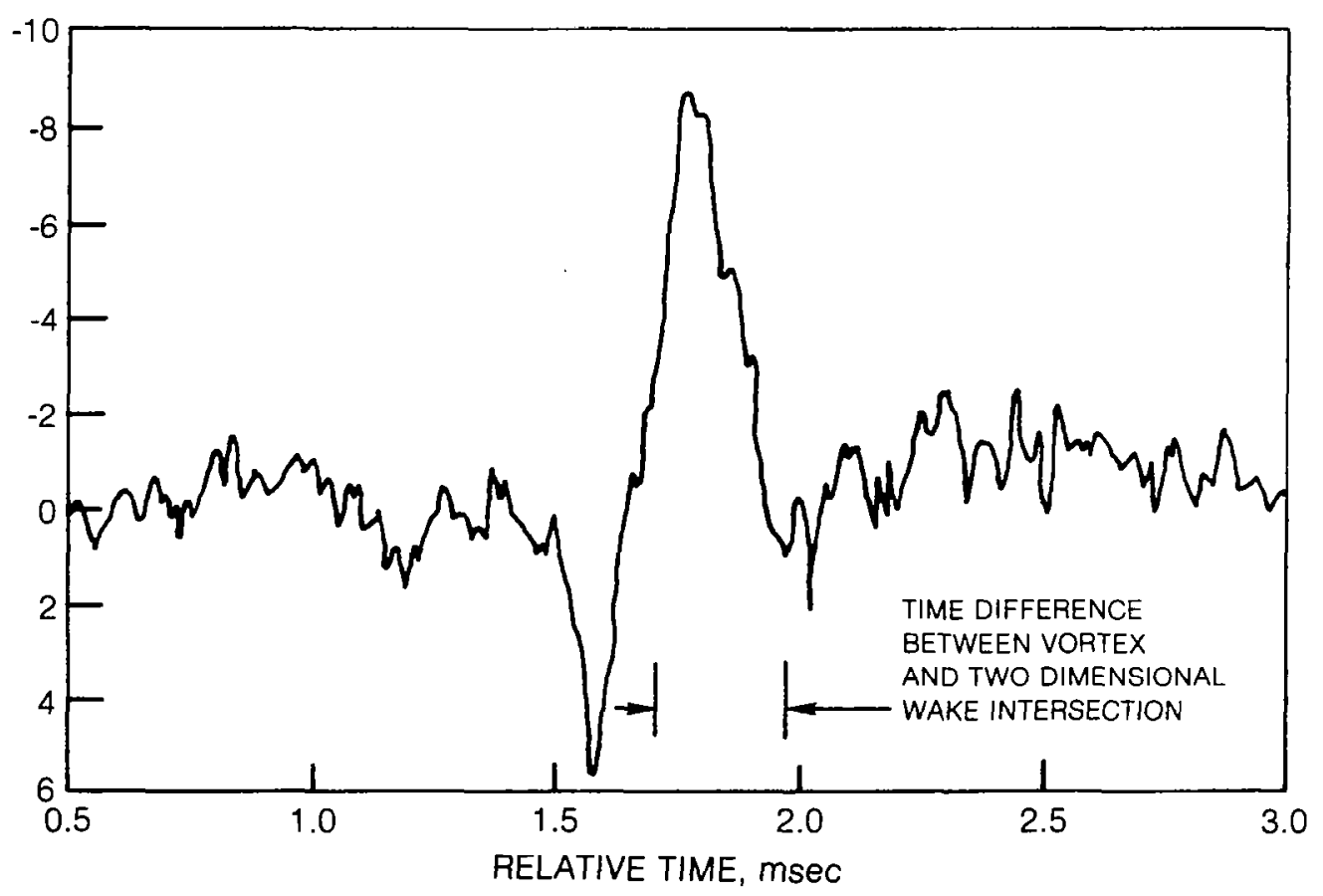

d) HIGH RESOLUTION TIME HISTORY OF BLADE VORTEX INTERACTION

Figure 26 - Concluded 


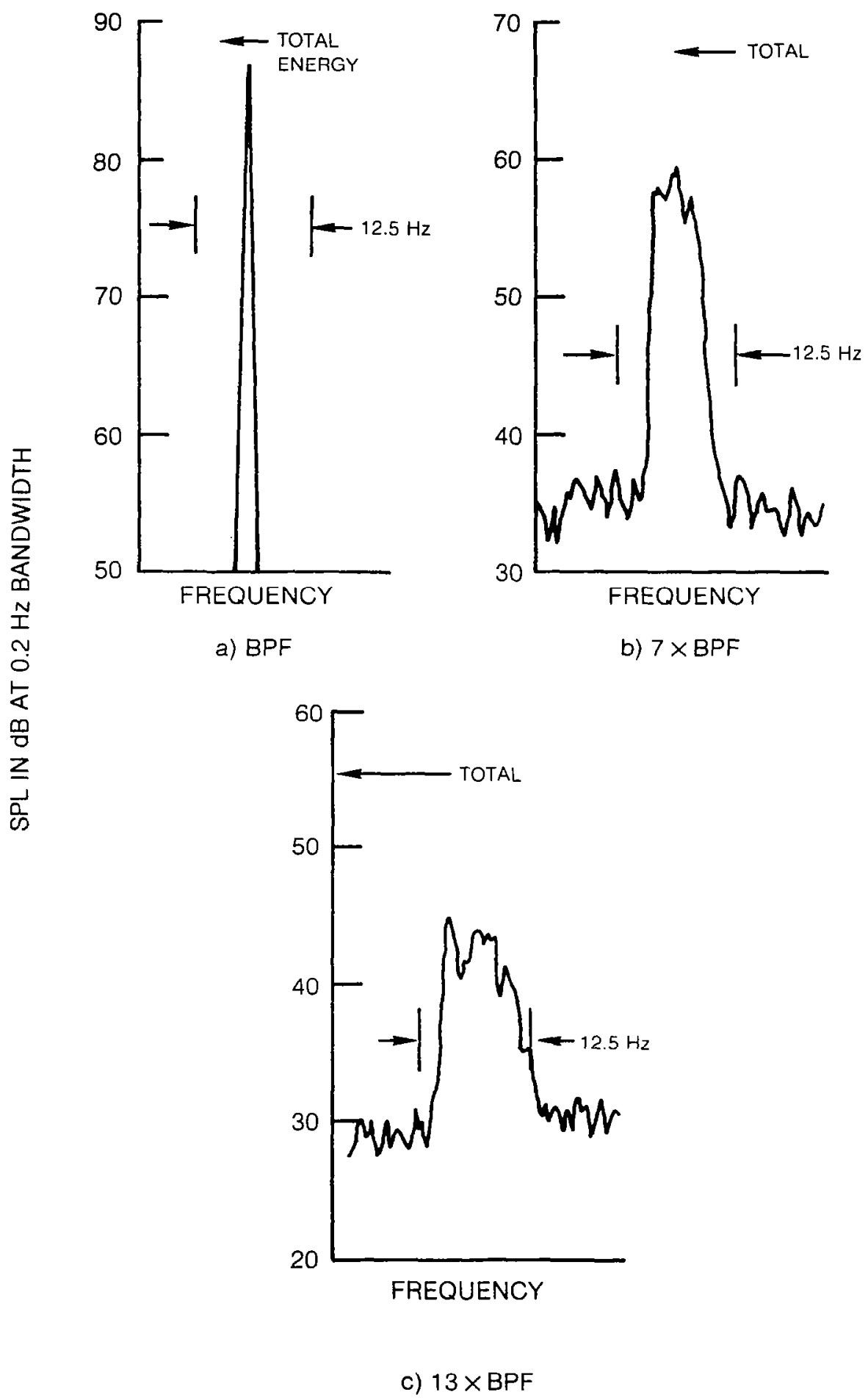

Figure 27 - High Resolution Spectrum of Narrowband Random Tones in Acoustic Spectrum, Test Condition B, $\theta_{O}=120^{\circ}, \phi_{O}=180^{\circ}$, Test Condition B 

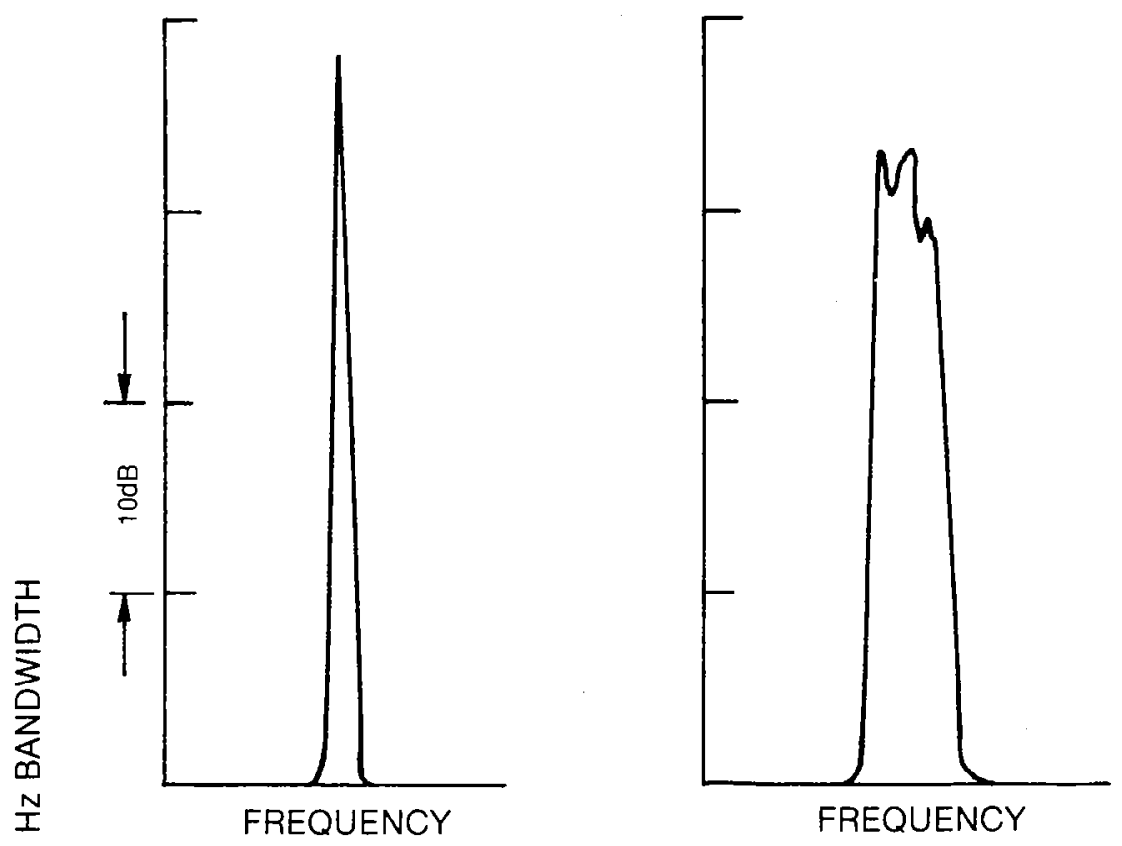

$\cong \quad$ a) SHAFT FUNDAMENTAL

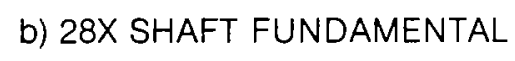

齐

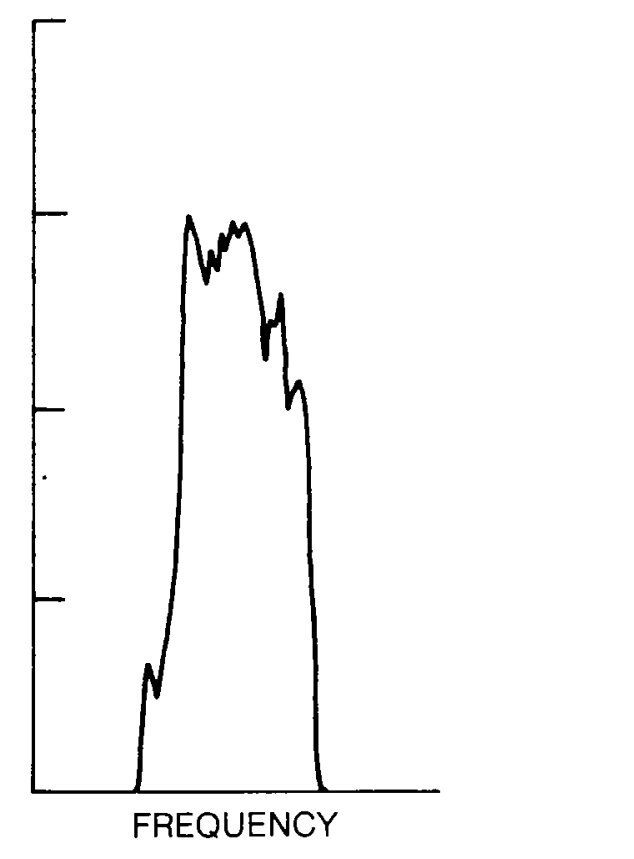

c) 52X SHAFT FUNDAMENTAL

Figure 28 - High Resolution Spectrum of Shaft Rotation Time Marker Signal, Test Condition B 


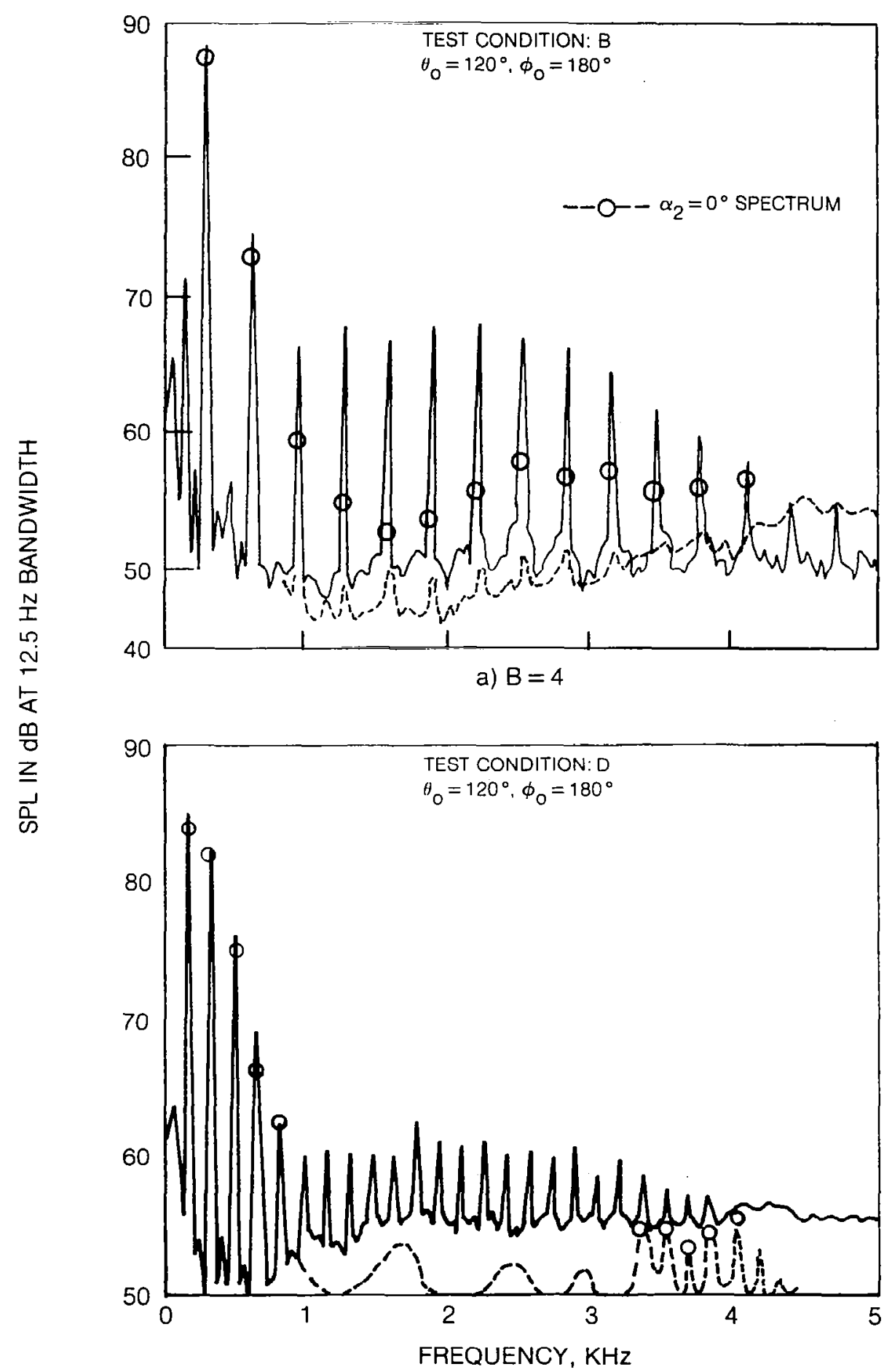

b) $B=2$

Figure 29 - Effect of Blade Number on Discrete Tone Spectrum 


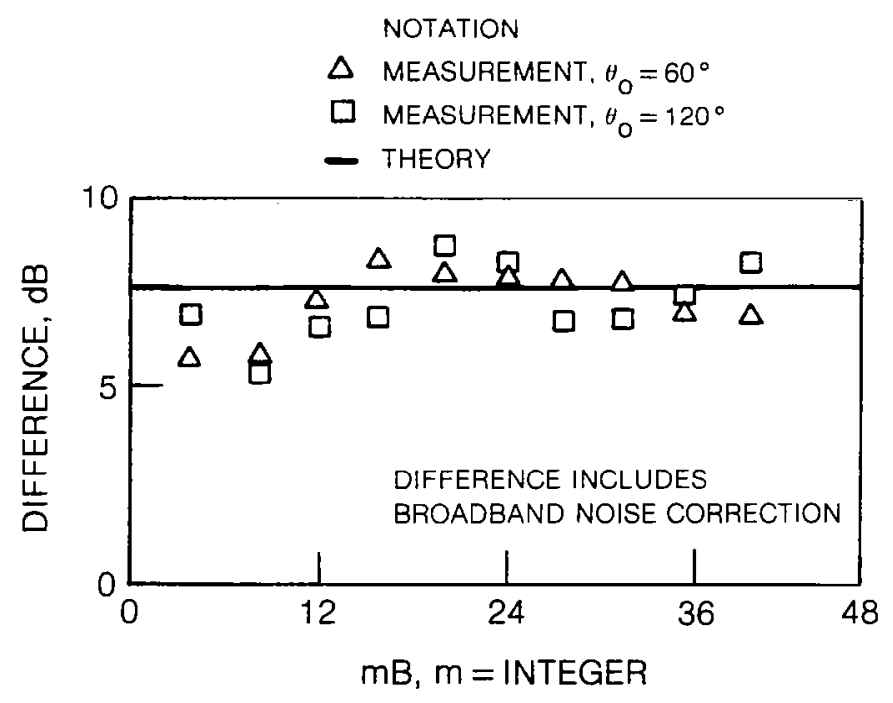

c) DIFFERENCE. BETWEEN SHAFT HARMONICS FOR $B=4$ AND $B=2$ AT TWO RADIATION ANGLES, $\theta_{0}$

Figure 29 - Concluded 


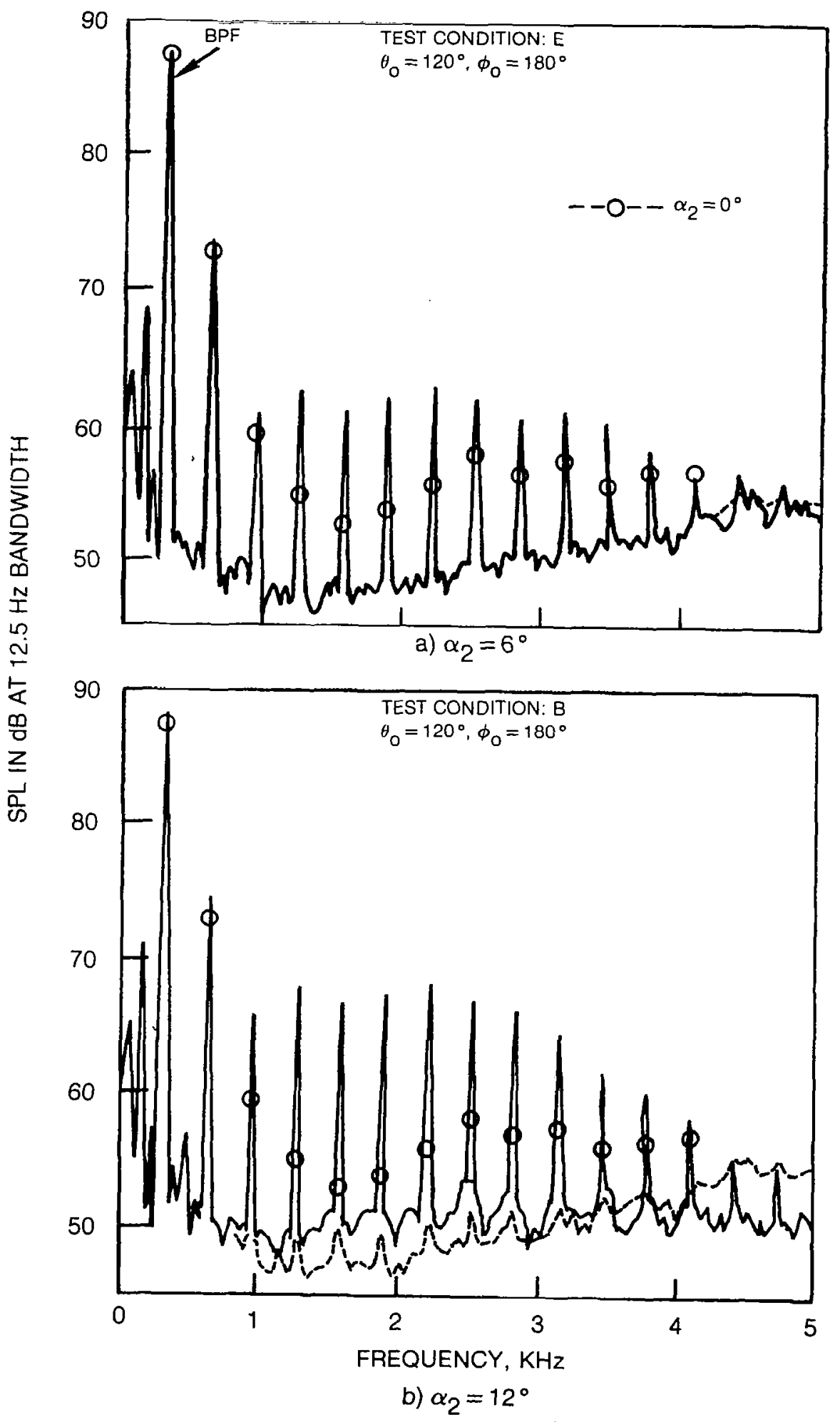

Figure 30 - Effect of Vortex Strength on Discrete Tone Spectrum 


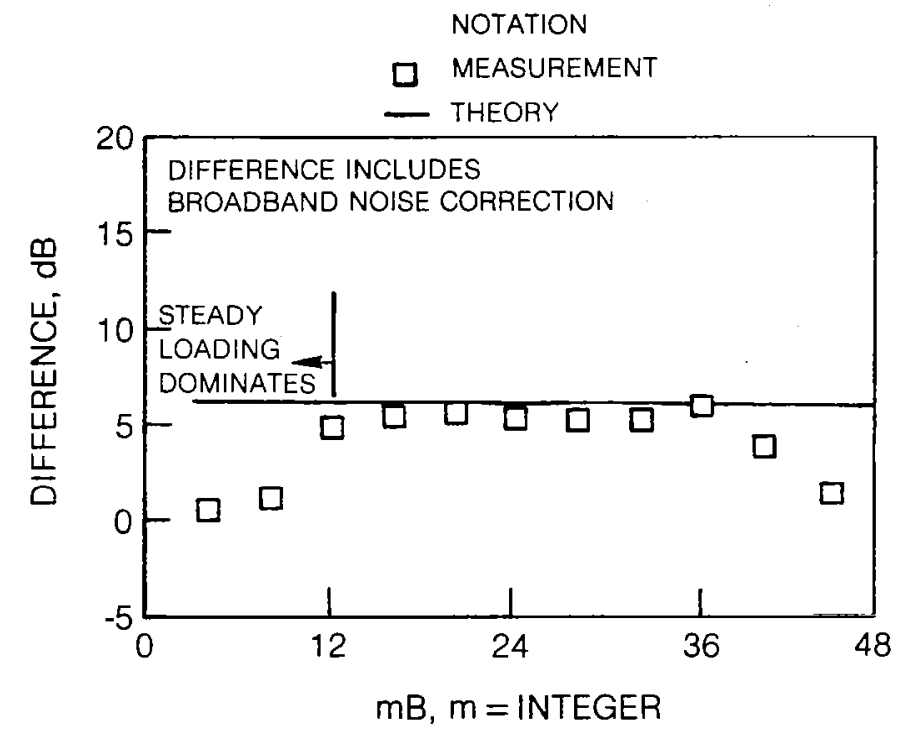

c) DIFFERENCE BETWEEN SHAFT

HARMONICS AT $\alpha_{2}=6^{\circ}$ AND $\alpha_{2}=12^{\circ}$

Figure $30-$ Concluded 


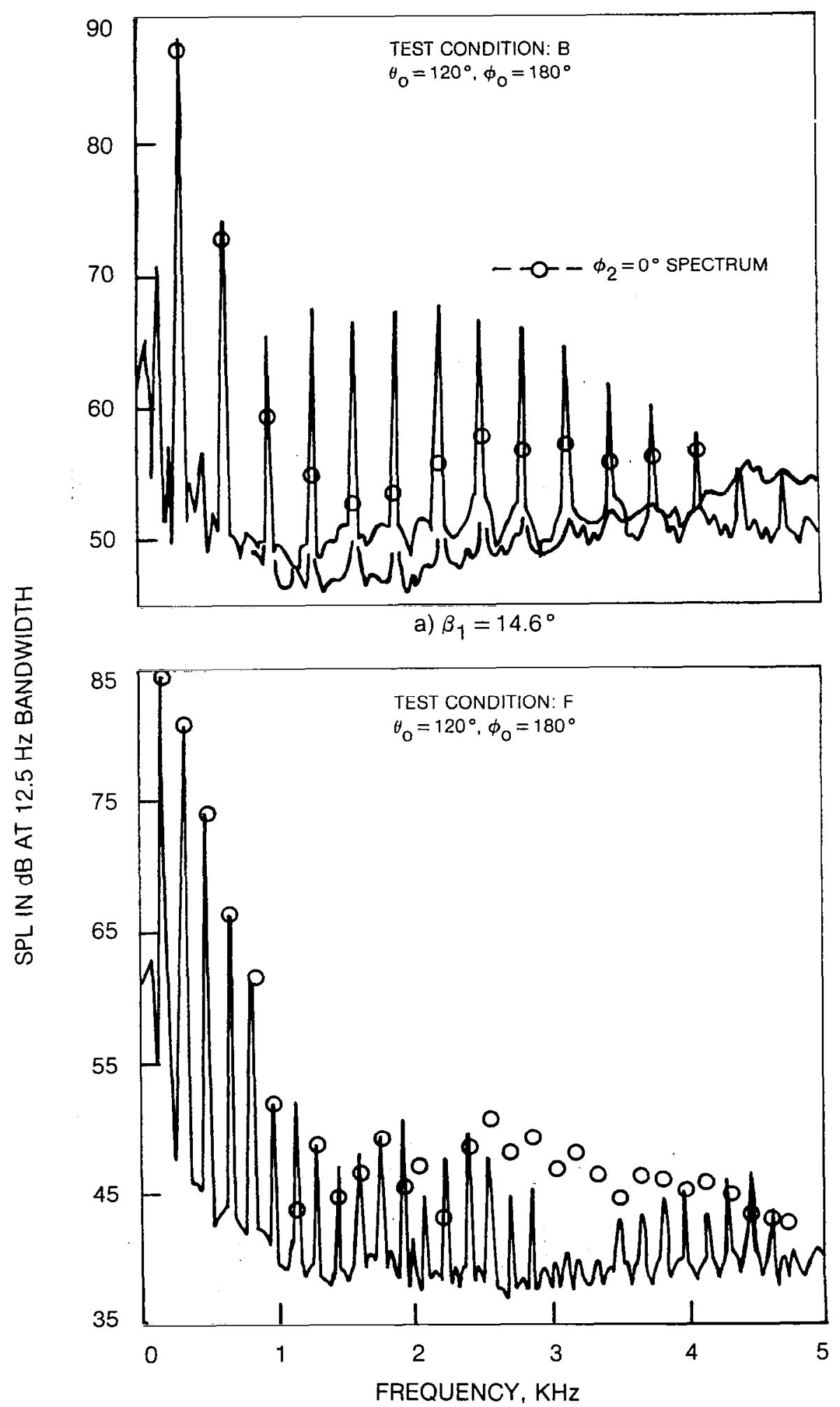

b) $\beta_{1}=0^{\circ}$

Figure 31 - Significance of Vortex Intersection Angle 


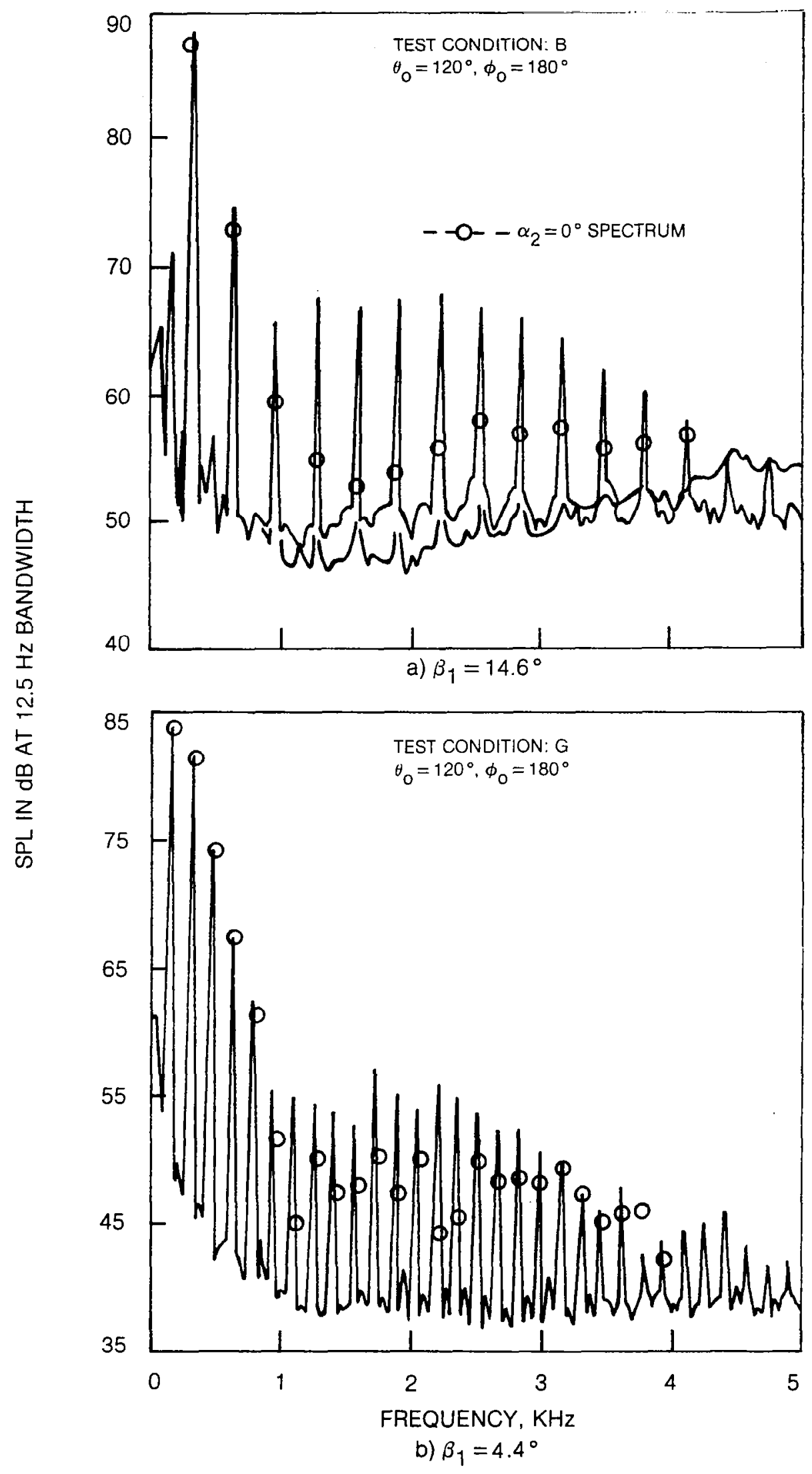

Figure 32 - Vortex Intersection Angle Dependence 


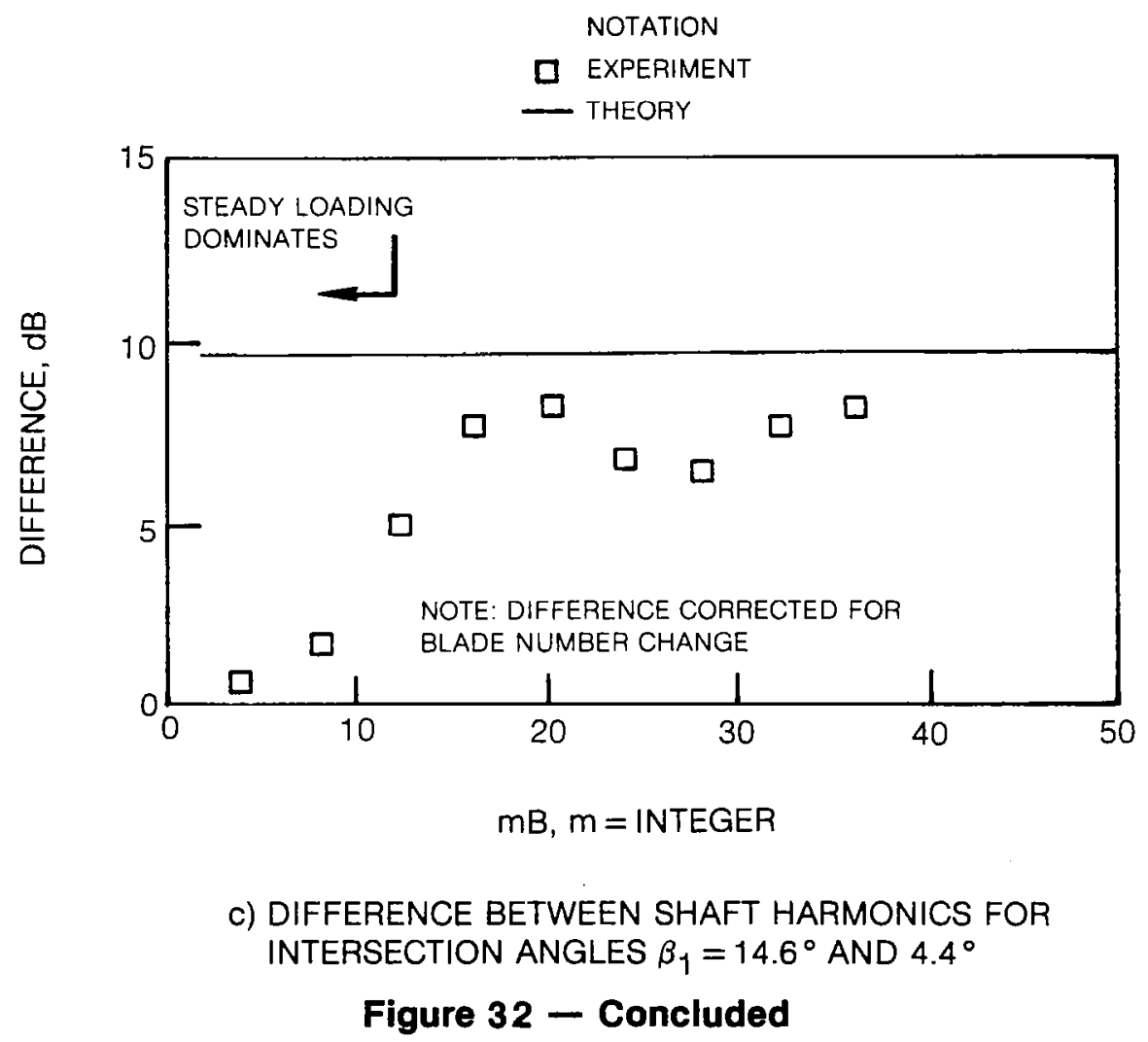




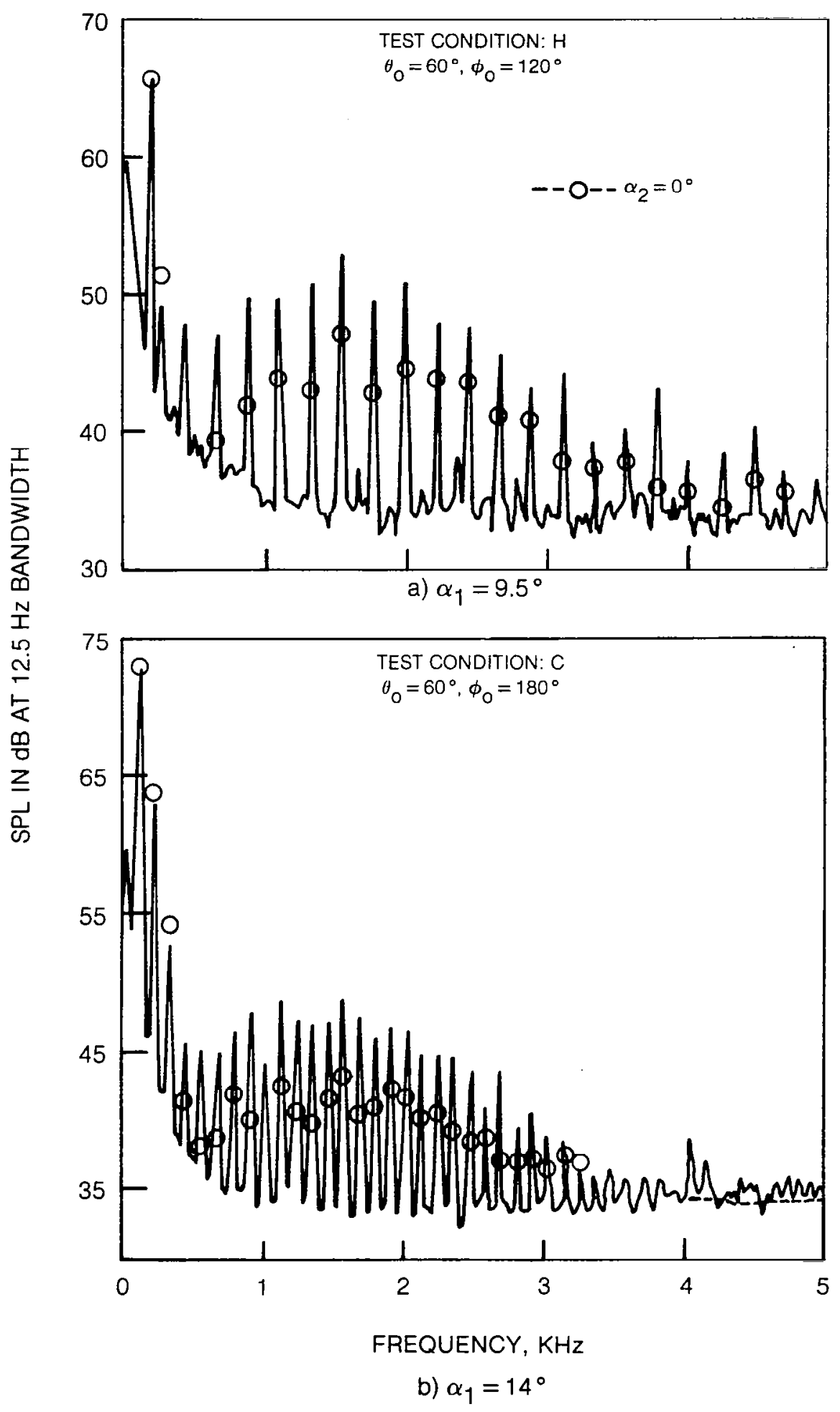

Figure 33 - Blade Pitch Angle Dependence 


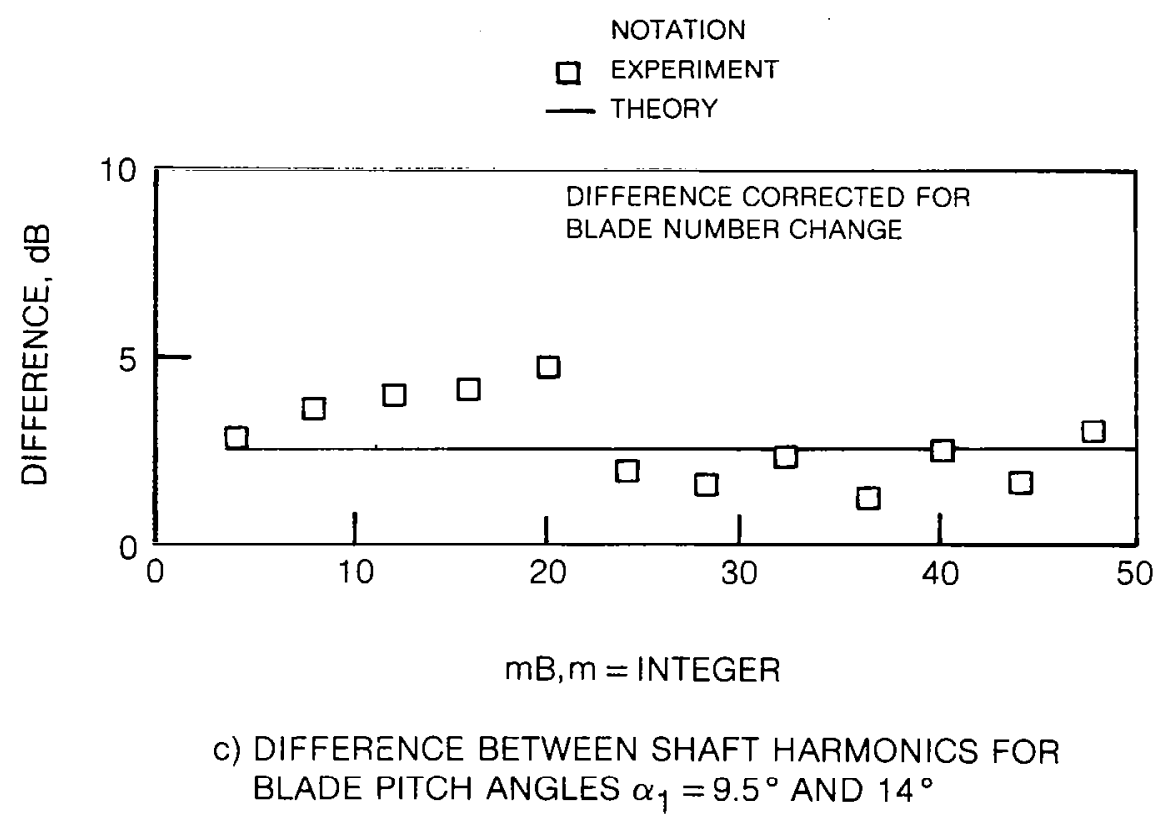

Figure 33 - Concluded 


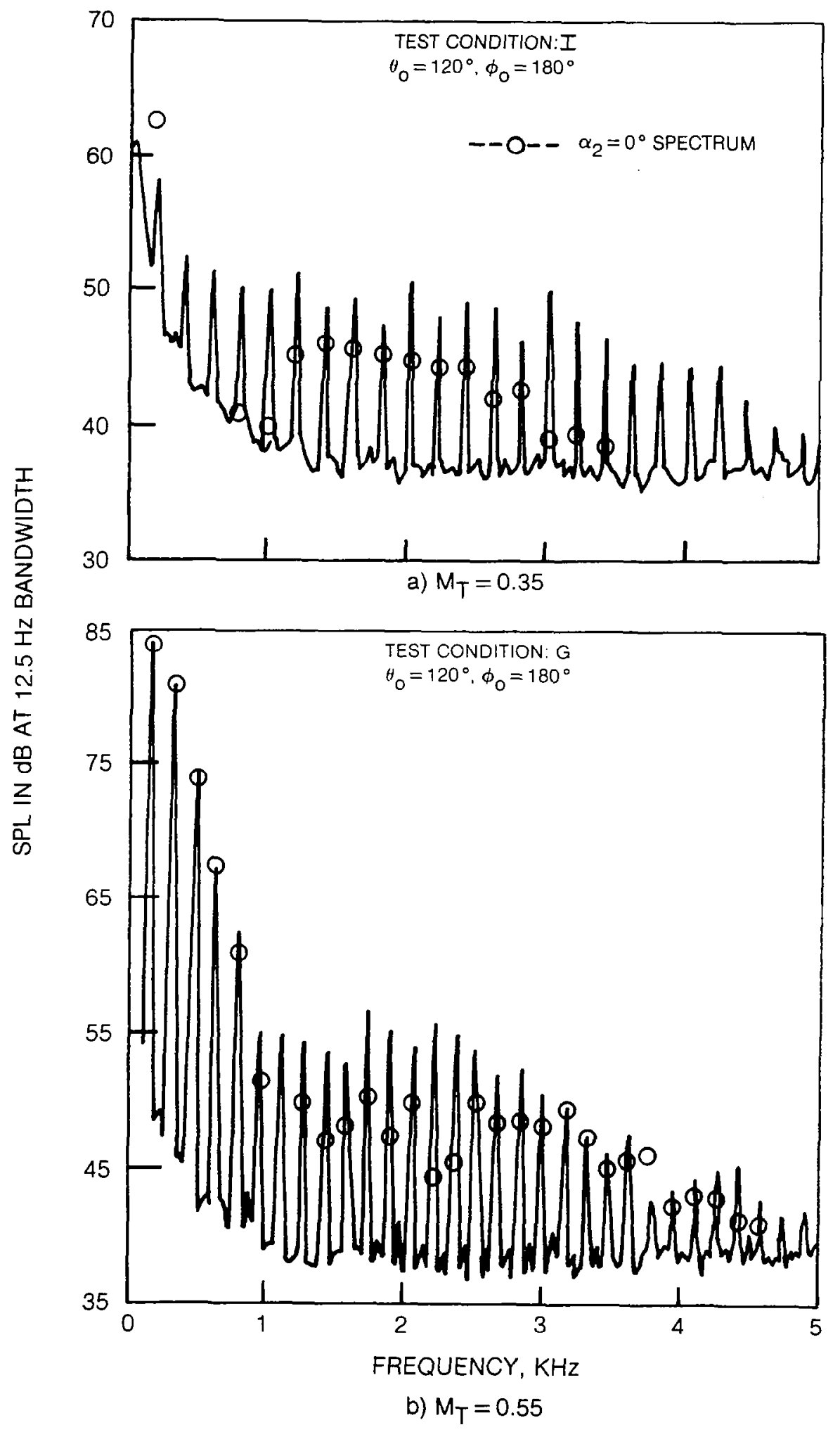

Figure 34 - Mach Number Dependence 


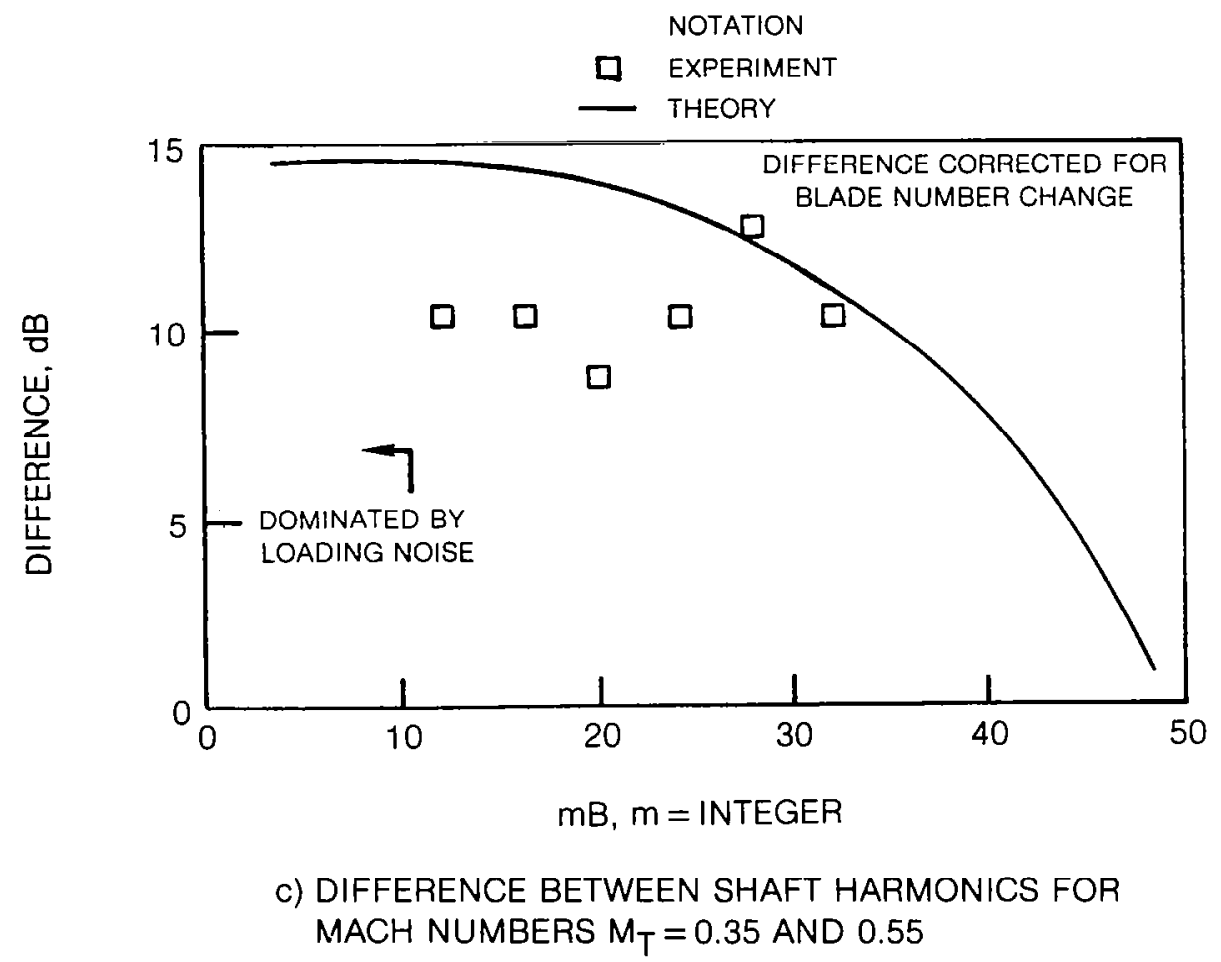

Figure $34-$ Concluded 


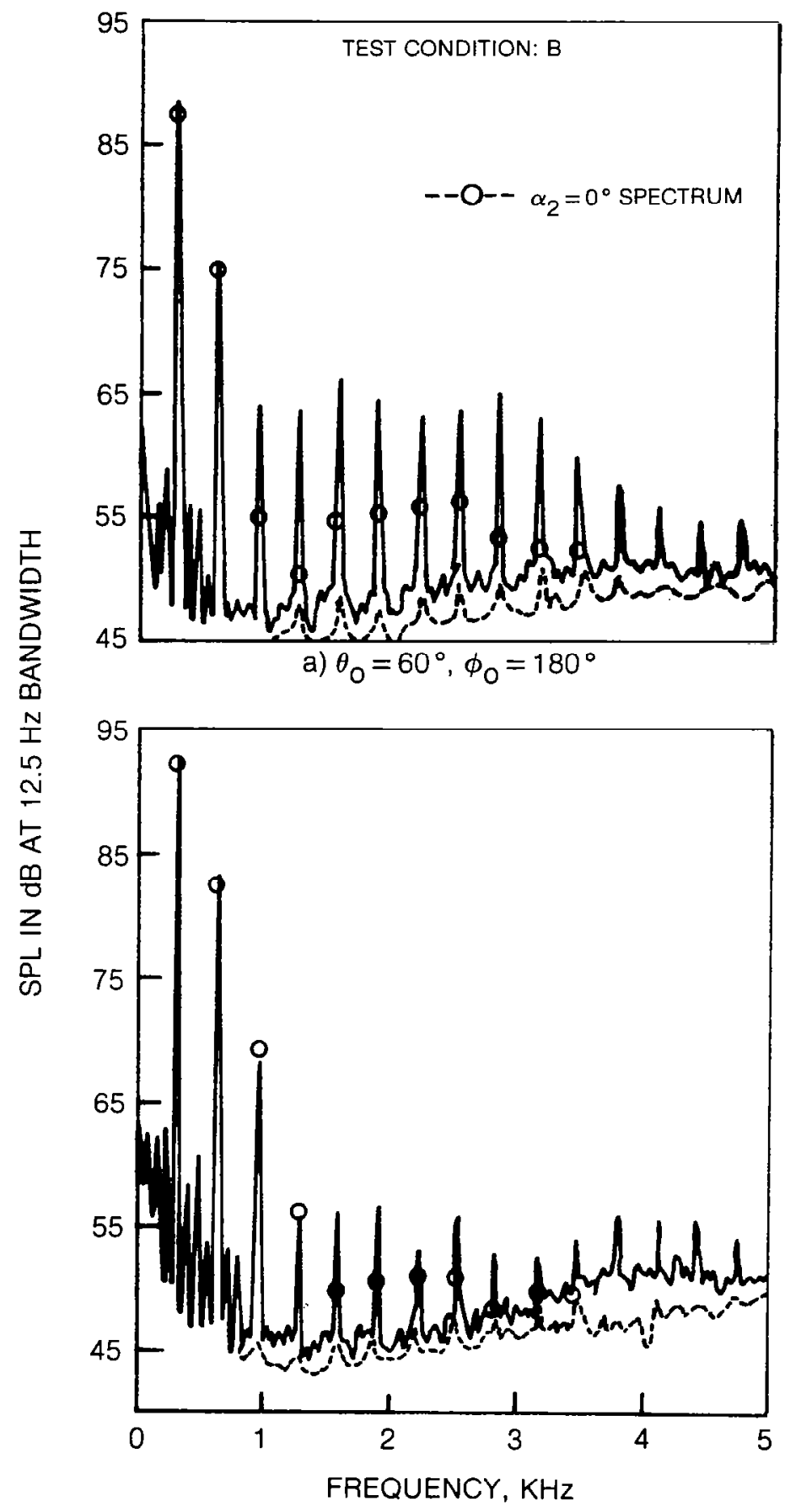

b) $\phi_{O}=80^{\circ}, \phi_{O}=180^{\circ}$

Figure 35 - Comparison of Spectra at Different Measurement Angles 


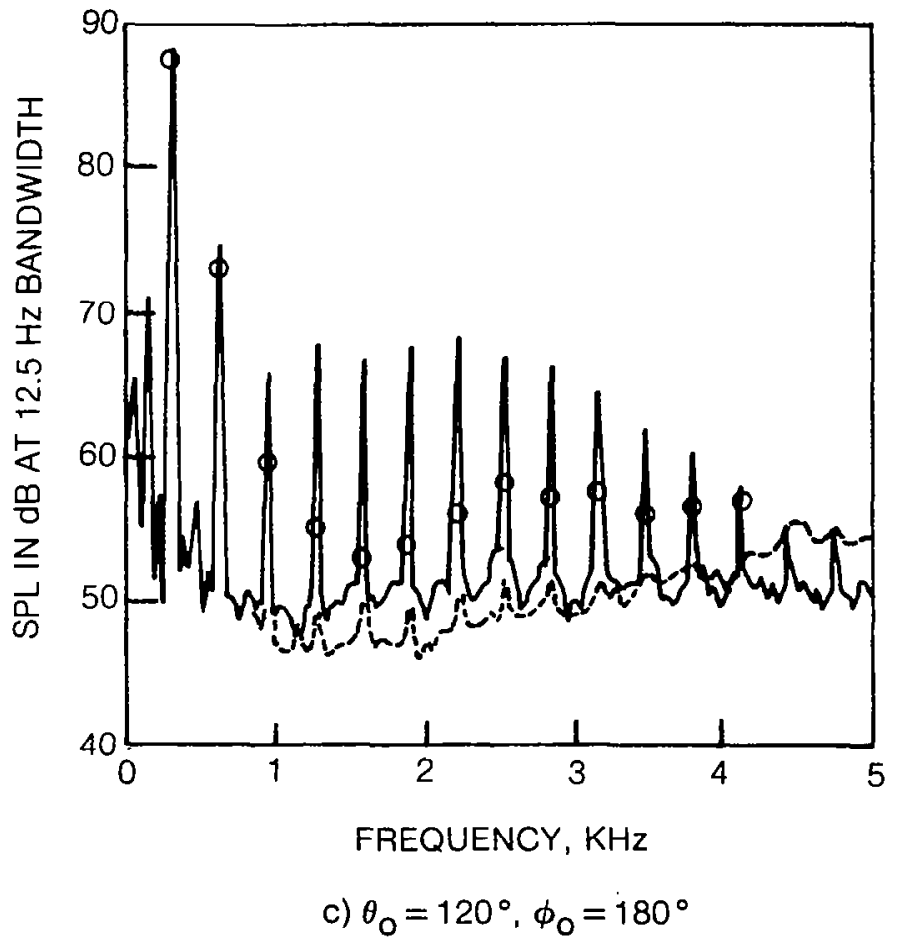

Figure 35 - Concluded 


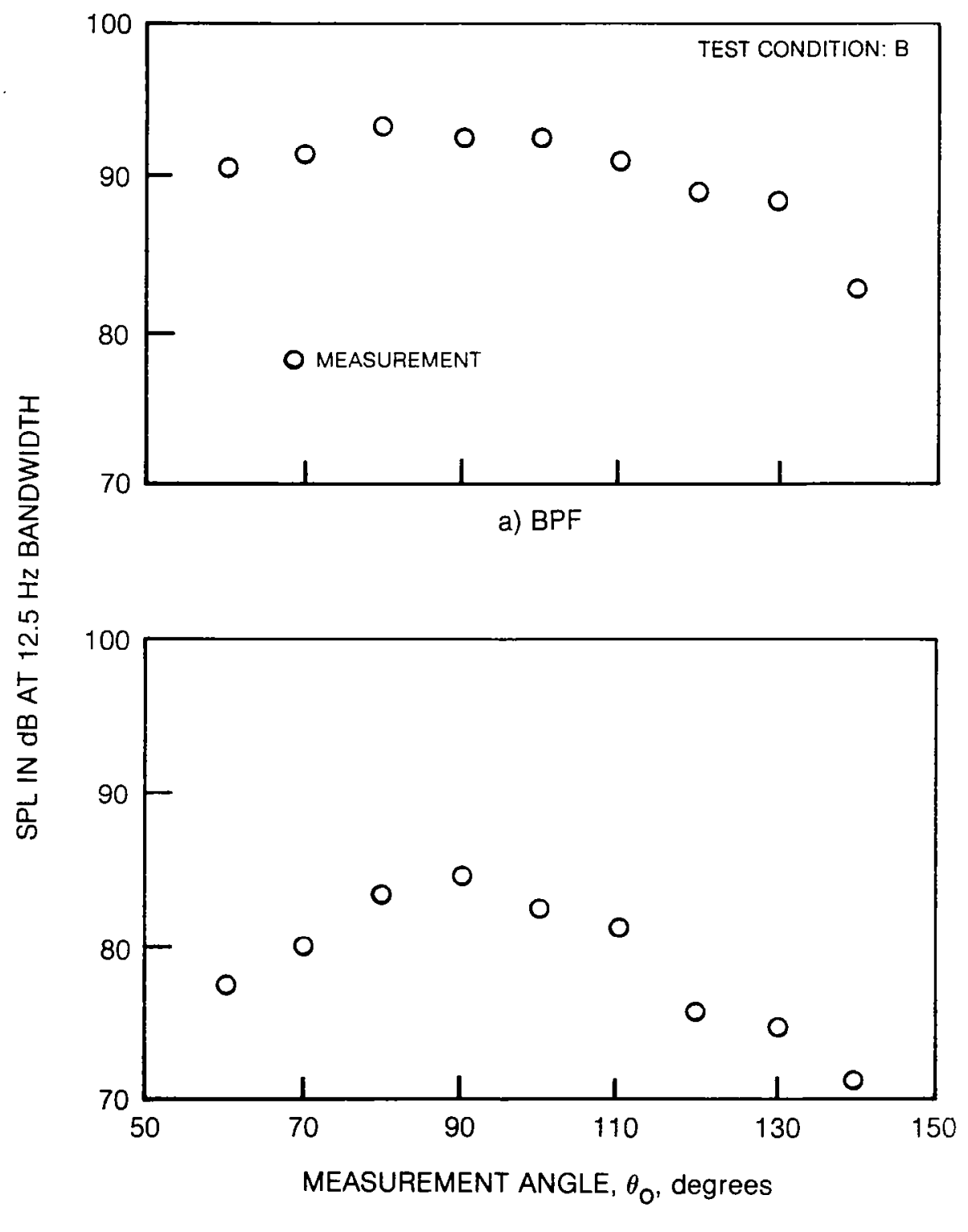

b) $2 \times \mathrm{BPF}$

Figure 36 - Tone Directivity Pattern 

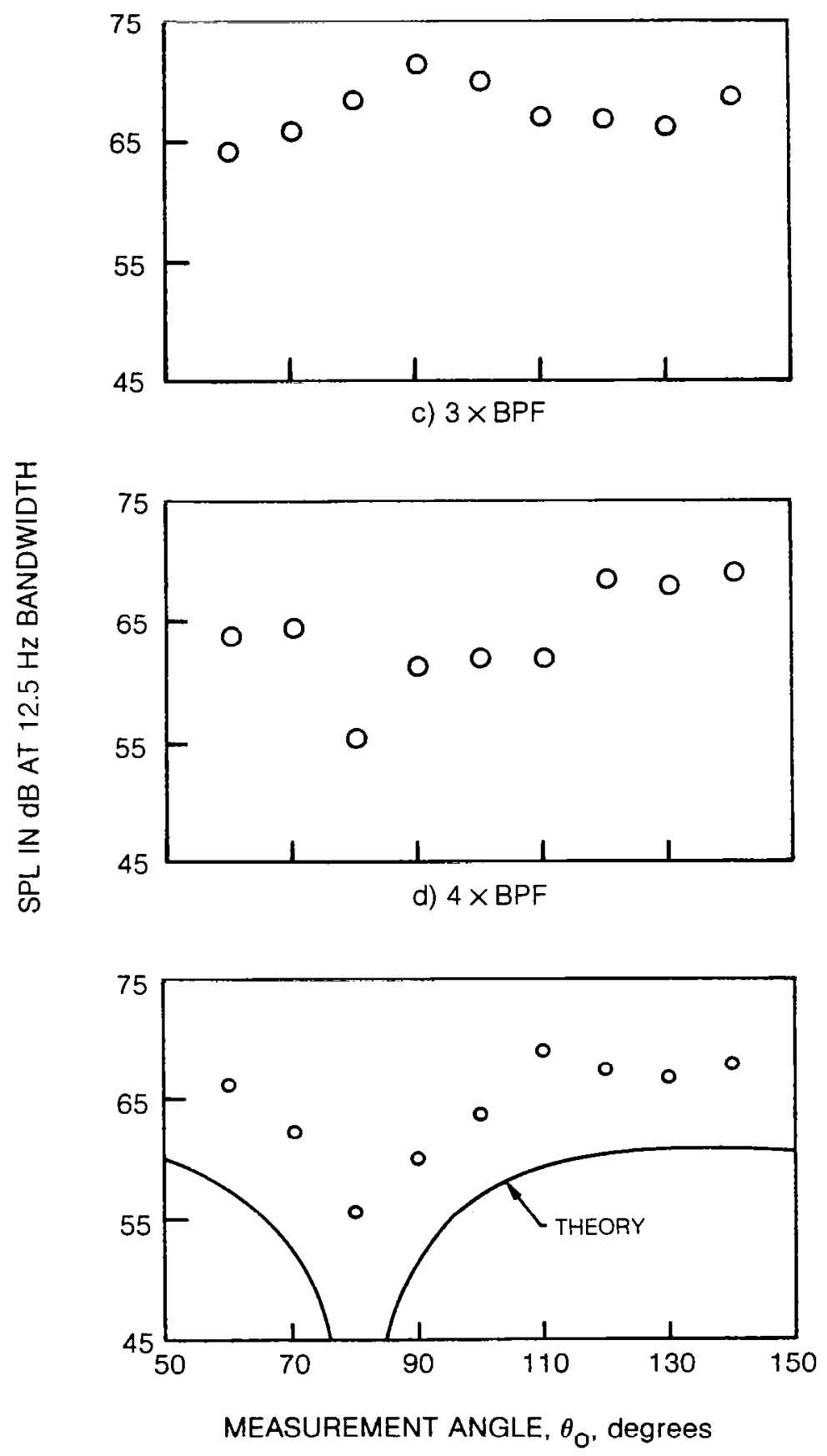

e) $5 \times B P F$

Figure $36-$ Continued 

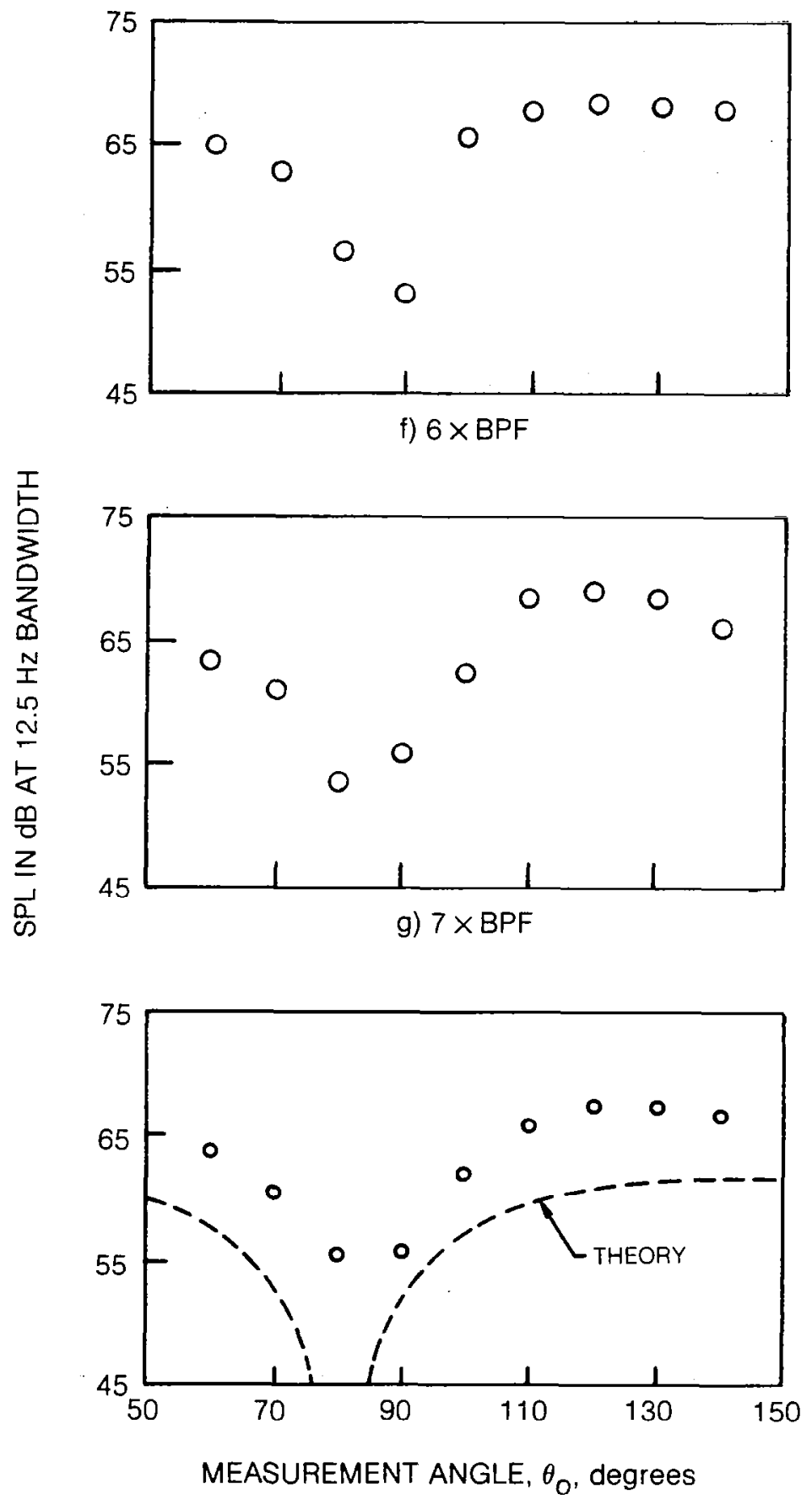

h) $8 \times \mathrm{BPF}$

Figure 36 - Continued 

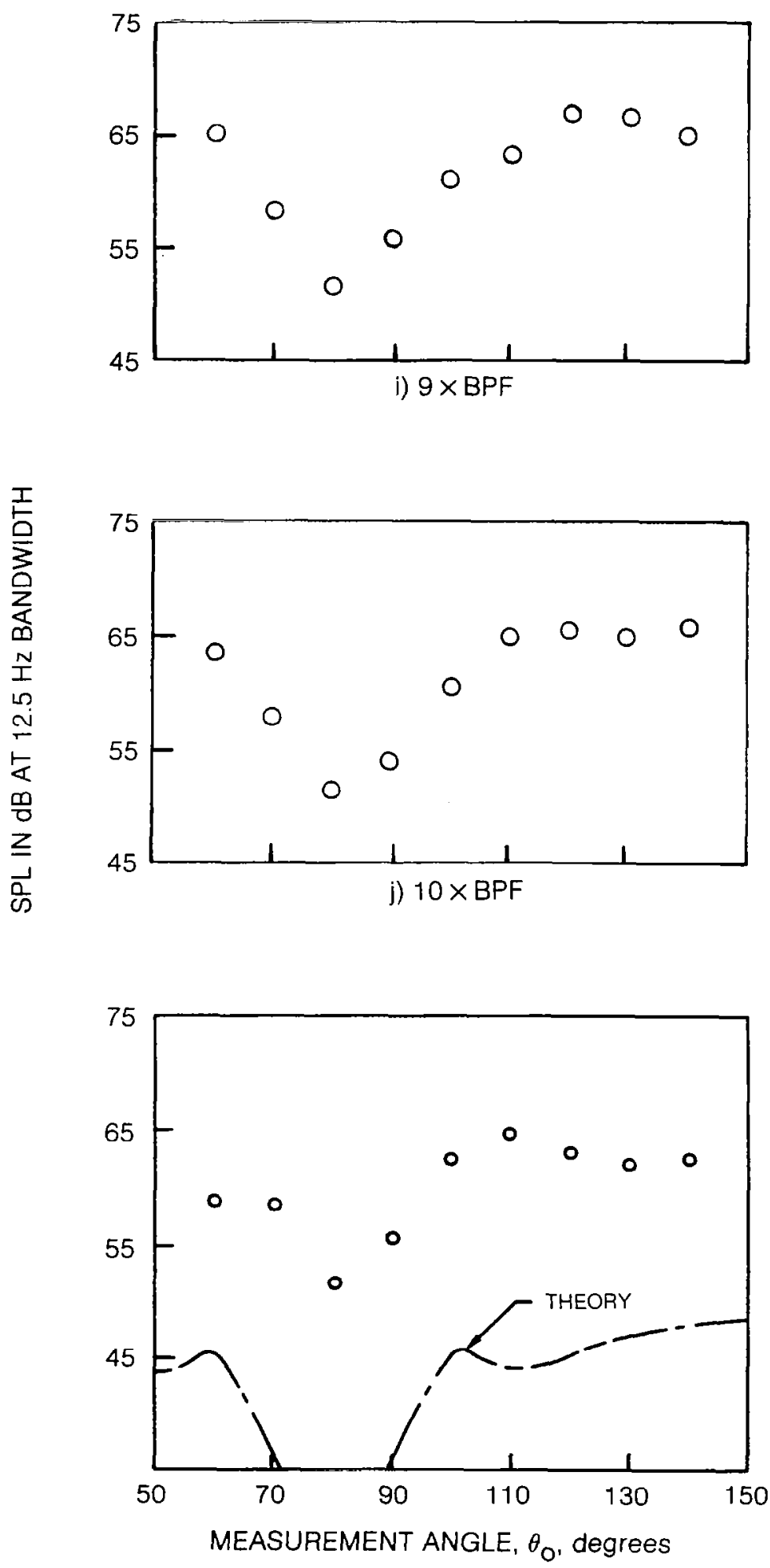

k) $11 \times \mathrm{BPF}$

Figure 36 - Continued 


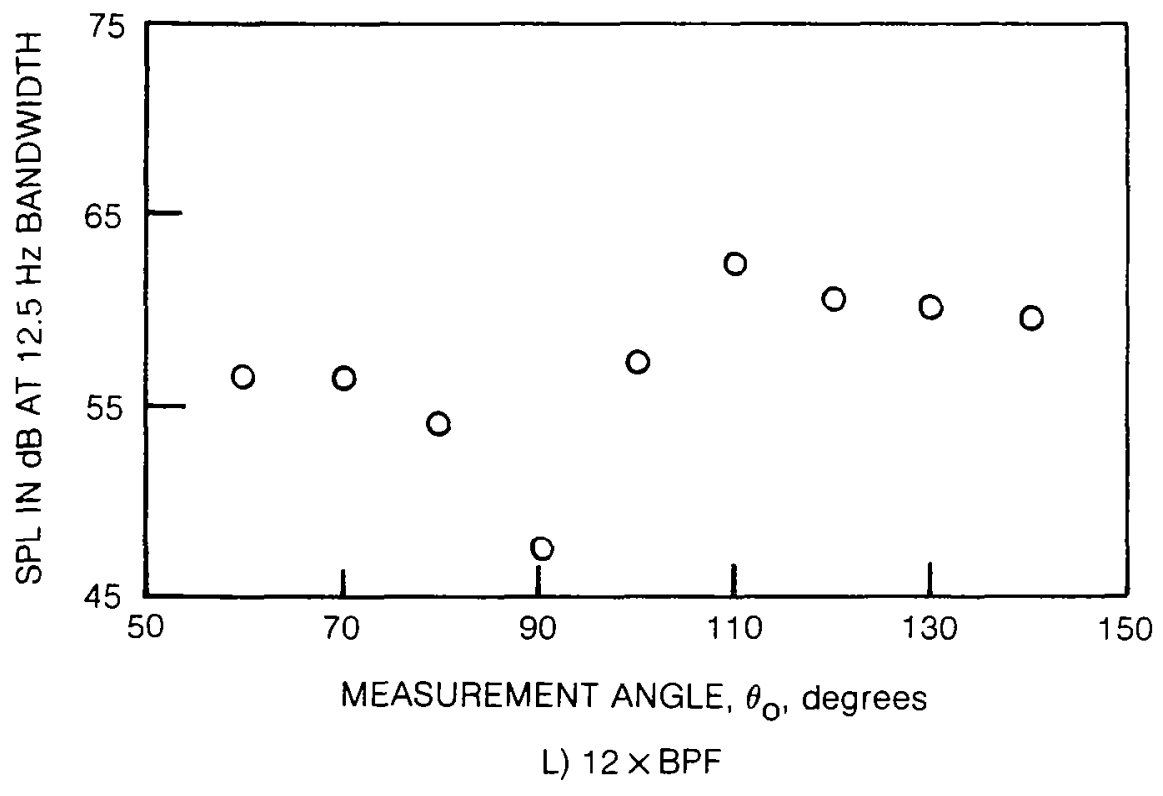

Figure 36 - Concluded 


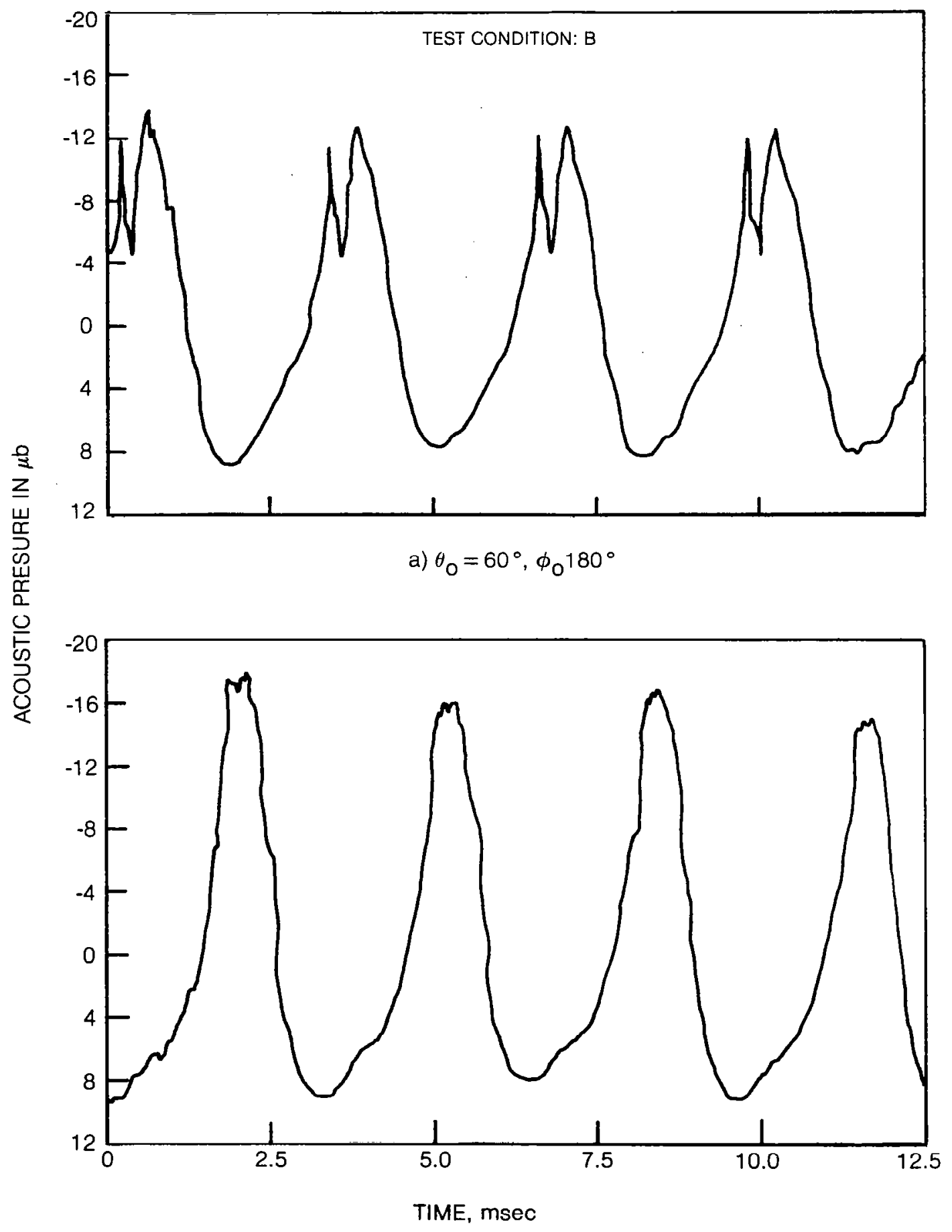

b) $\theta_{O}=90^{\circ}, \phi_{O}=180^{\circ}$

Figure 37 - Radiation Pattern of Combined Steady Loading and Blade Vortex Interaction Acoustic Signature 


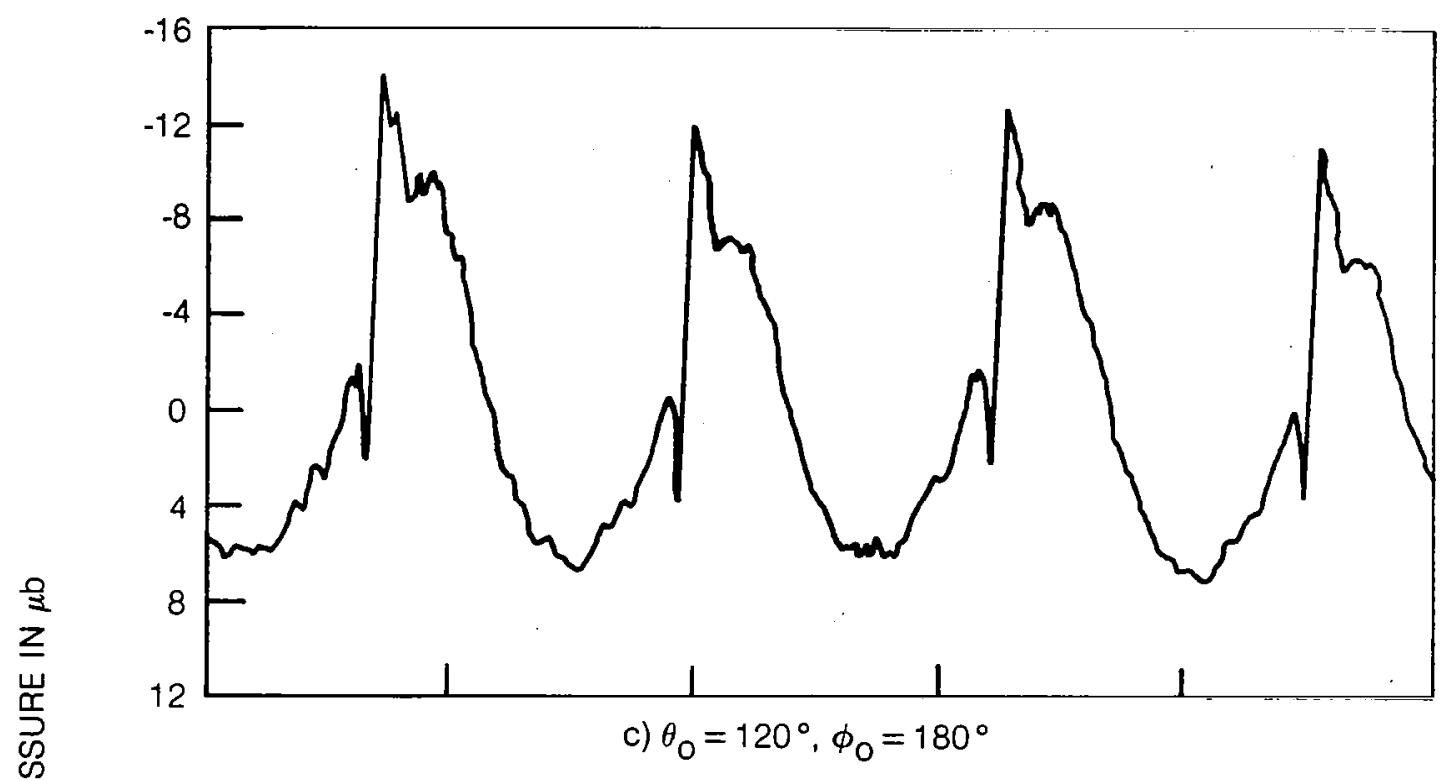

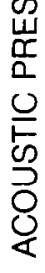

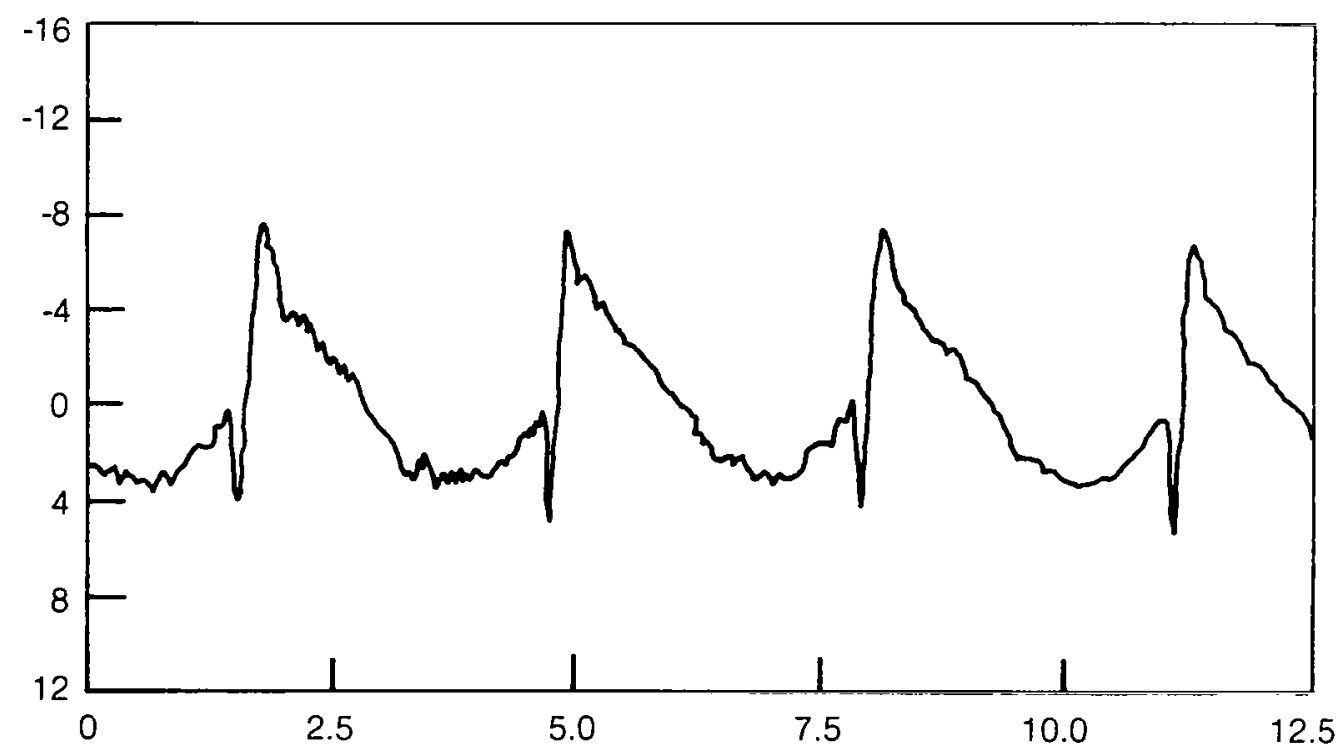

TIME, msec

d) $\theta_{0}=140^{\circ}, \phi_{0}=180^{\circ}$

Figure 37 - Concluded 

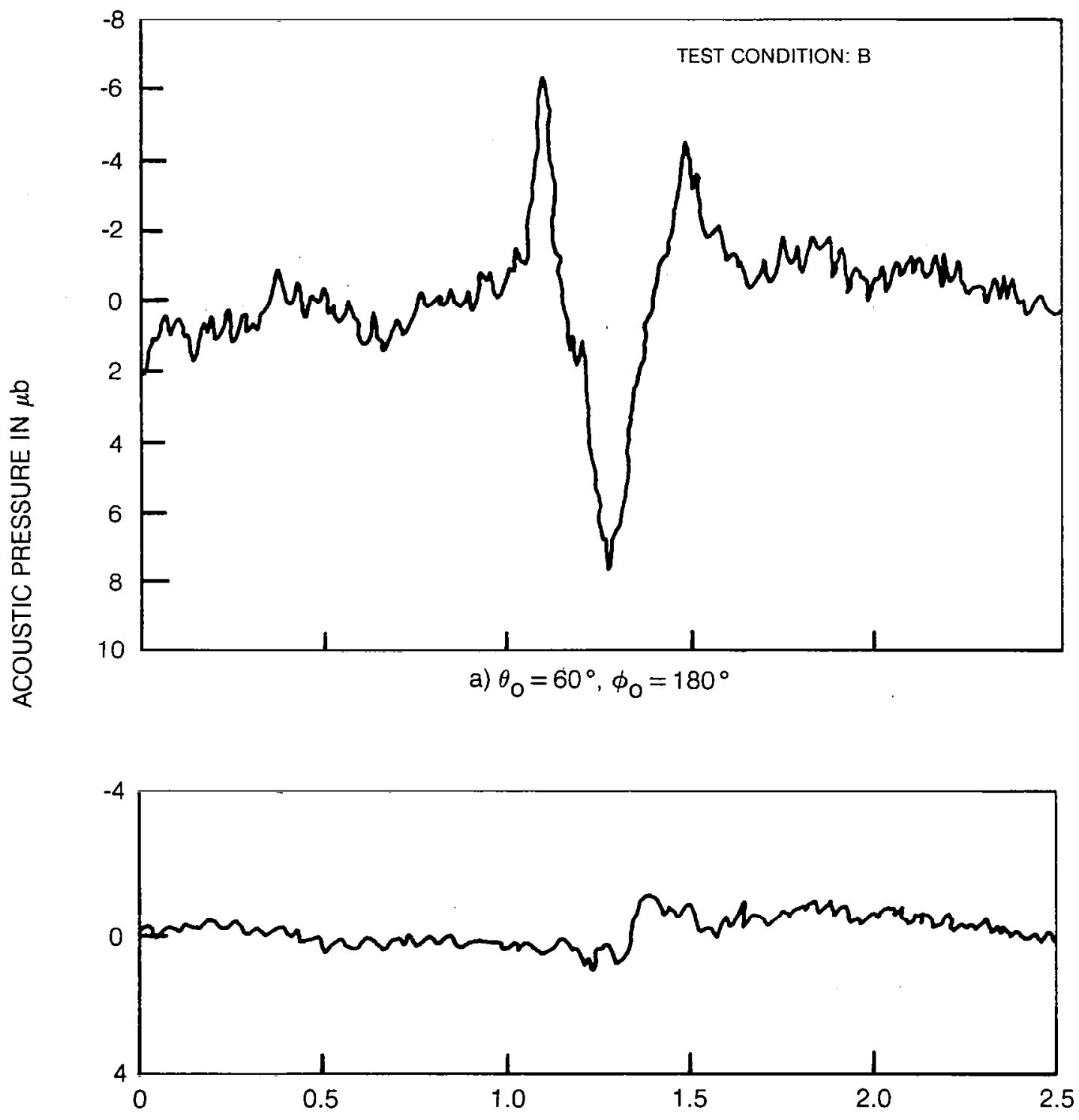

TIME, msec

b) $\theta_{o}=90^{\circ}, \phi_{O}=180^{\circ}$

Figure 38 - Radiation Pattern of Blade Vortex Interaction Acoustic Signature 


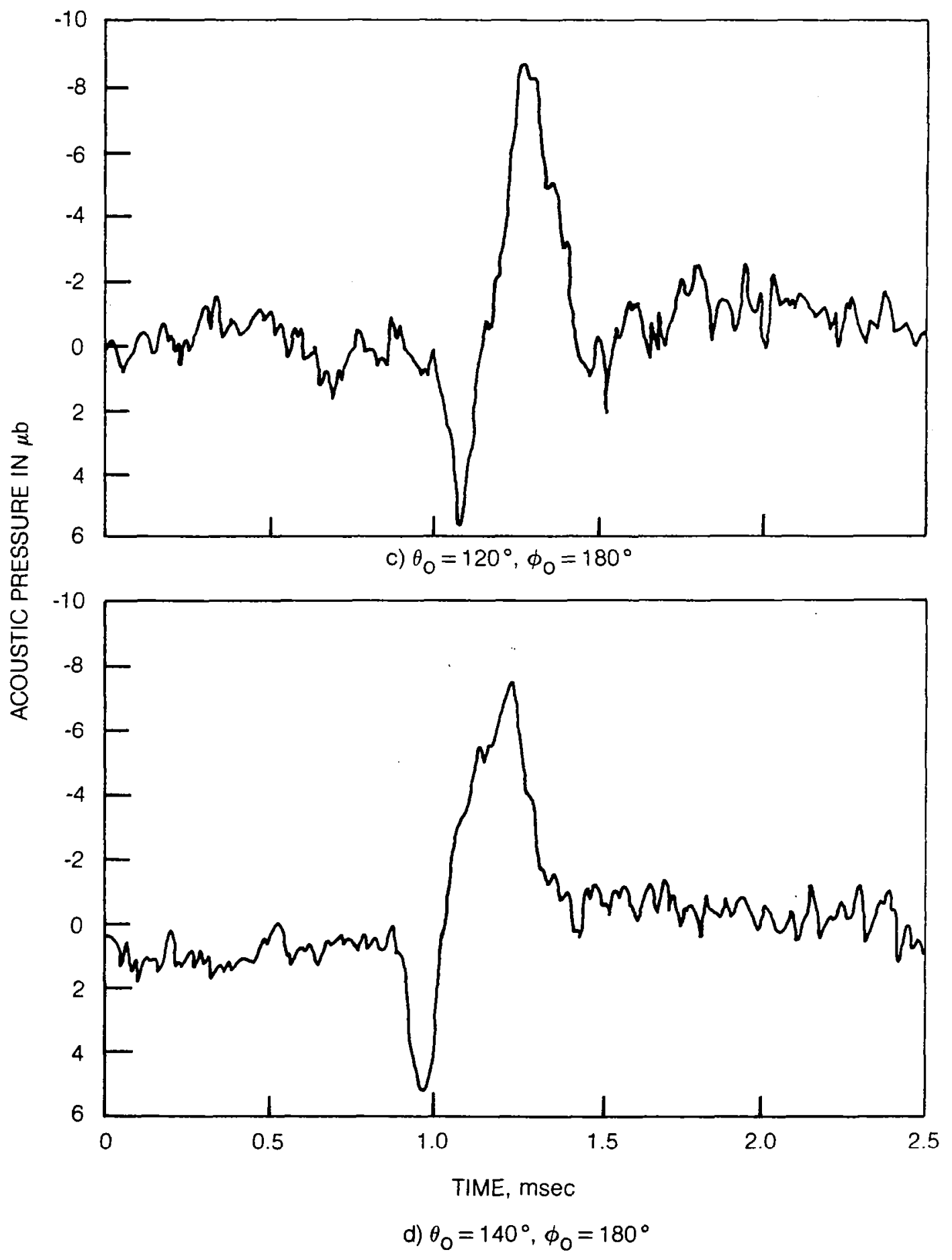

Figure 38 - Concluded 


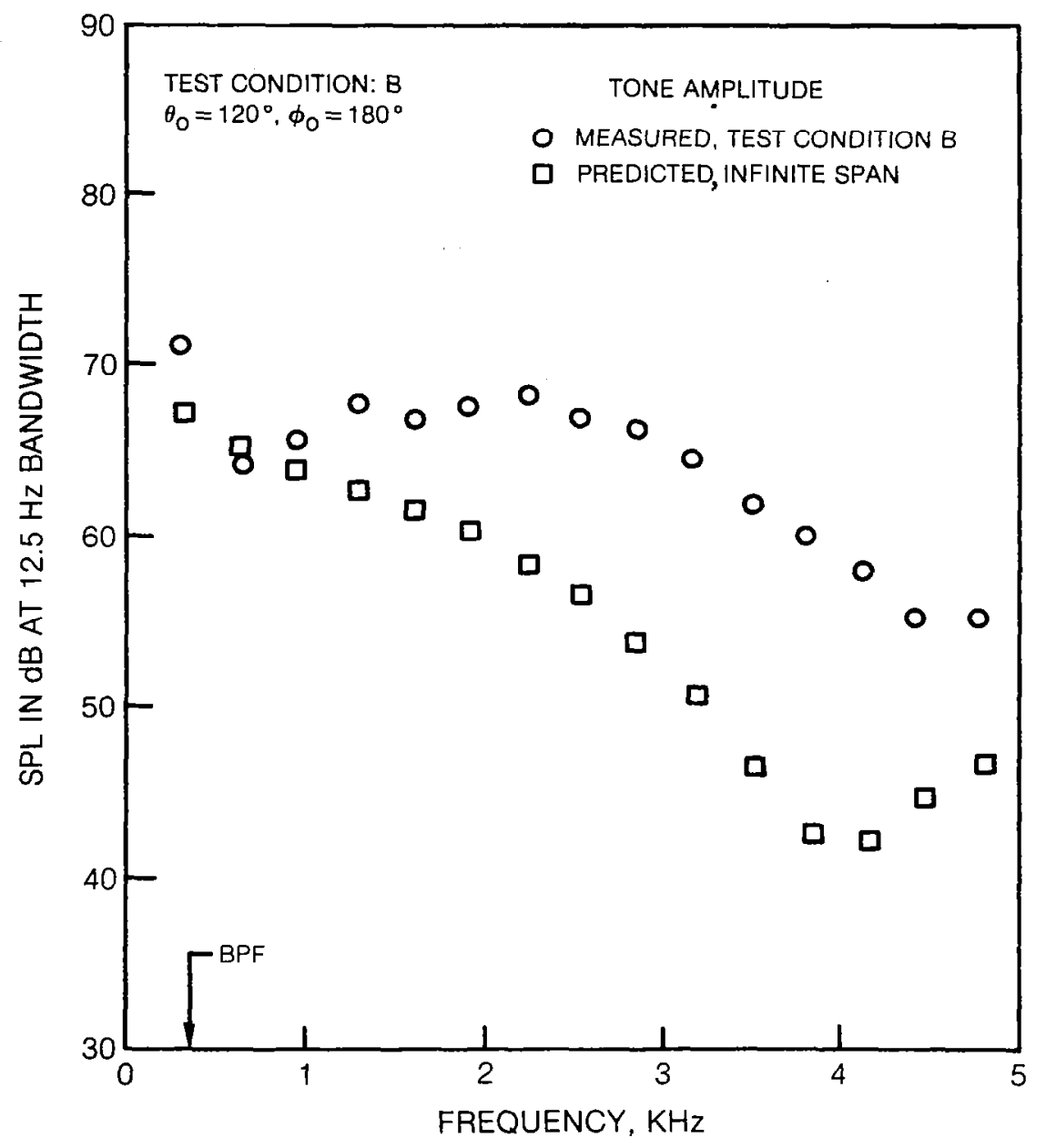

Figure 39 - Comparison of Measured and Predicted Tone Sound Pressure Levels 

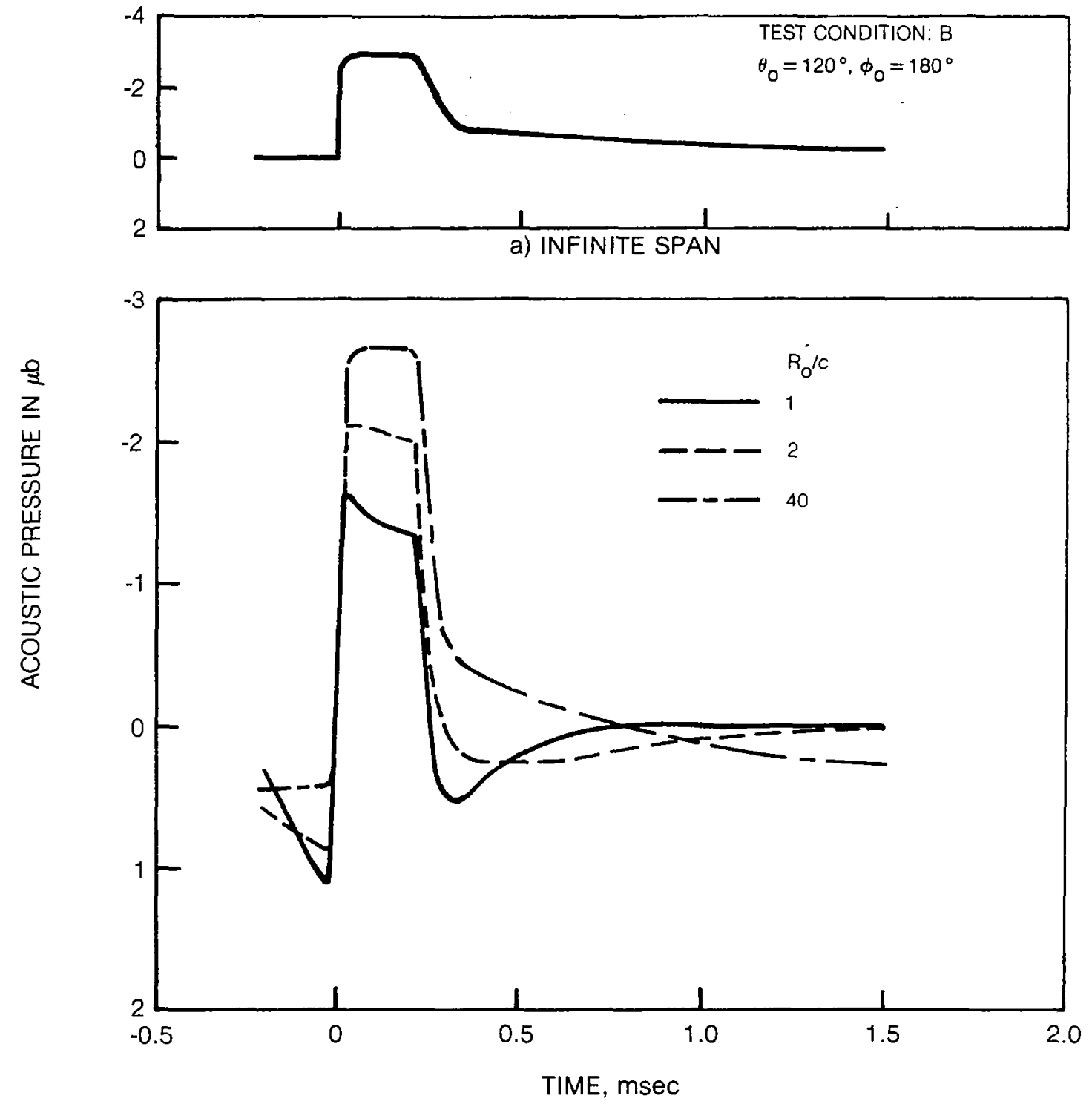

b) SIMULATION OF FINITE SPAN BY TERMINATING VORTEX

Figure 40 - Predicted Blade Vortex Interaction Acoustic Signature 


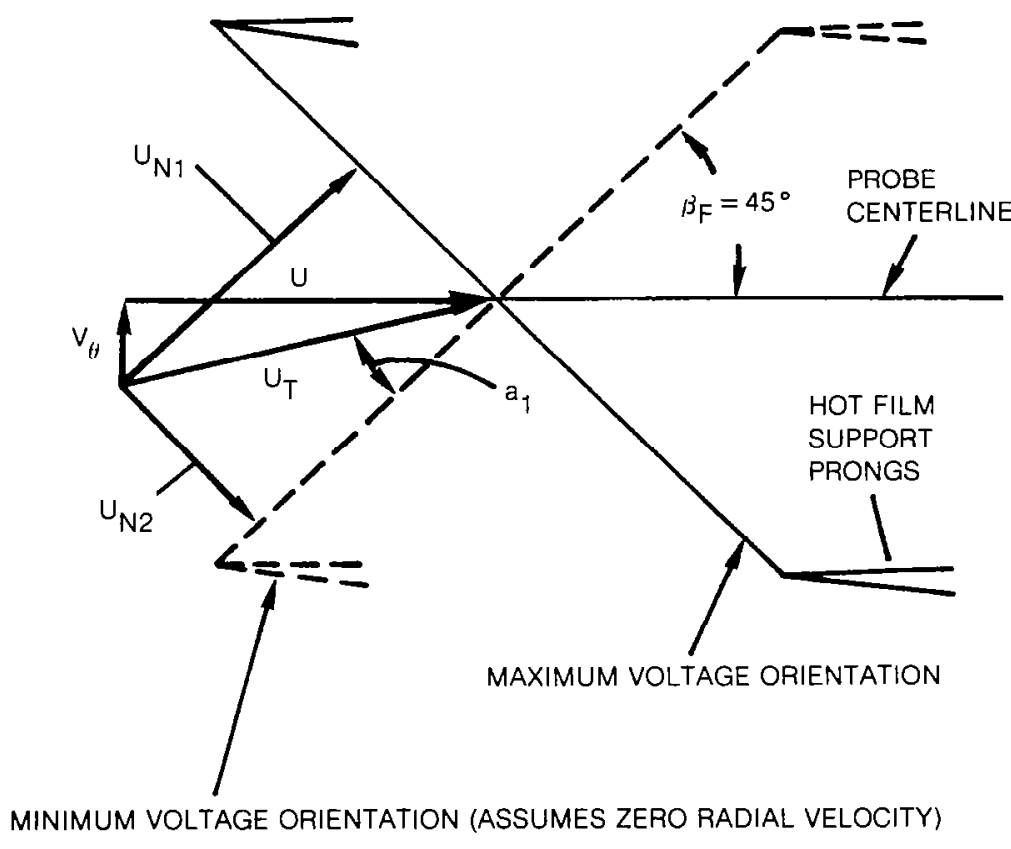

NOTE - HOT FILM SENSING ELEMENT LIES IN A PLANE PARALLEL TO $z$, y PLANE IN FIGURE 9

Figure A-1 - Velocity Components Sensed by Single Slanted Hot Film Probe 


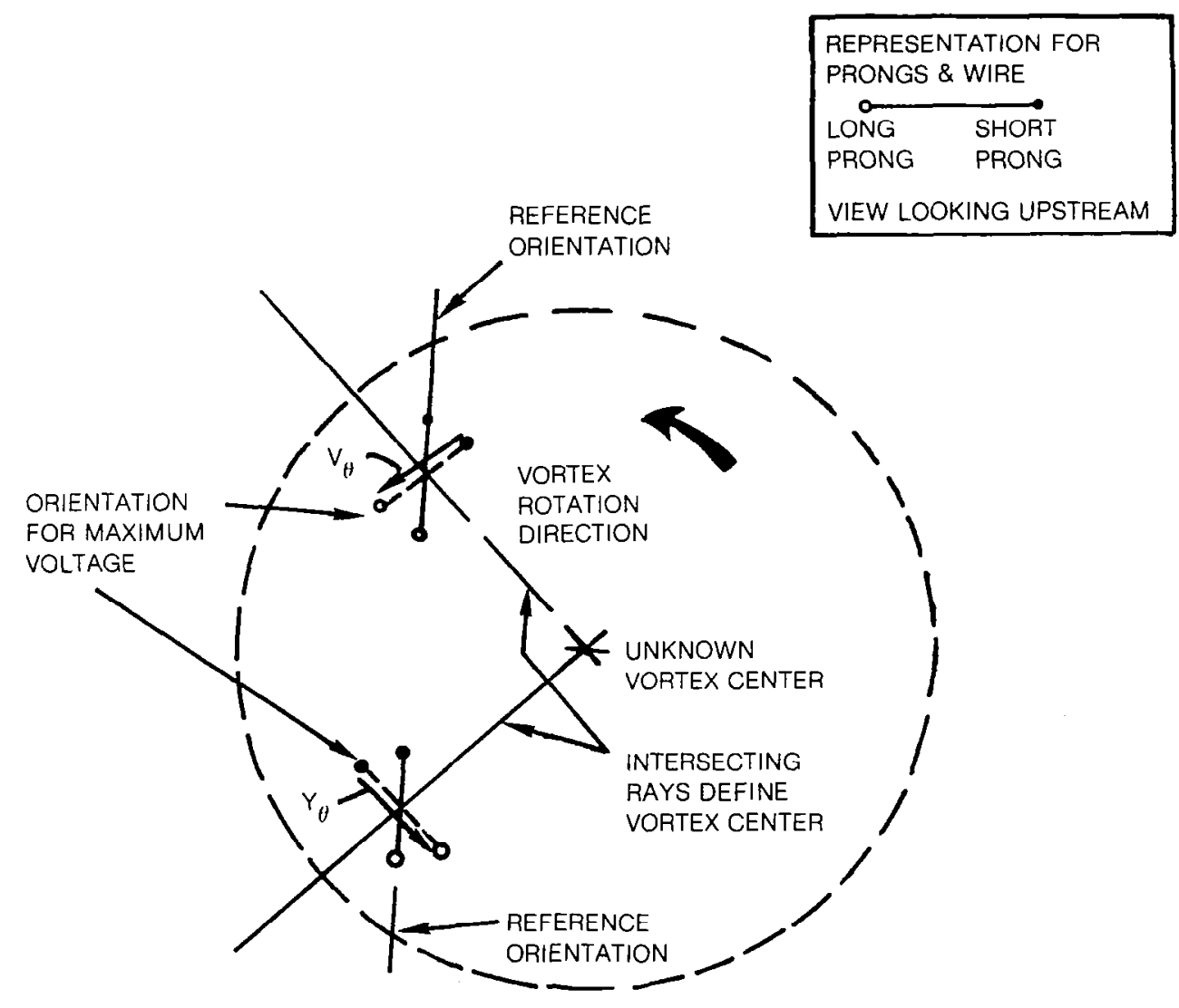

Figure A.2 - Schematic of Procedure Used to Identify Vortex Center 


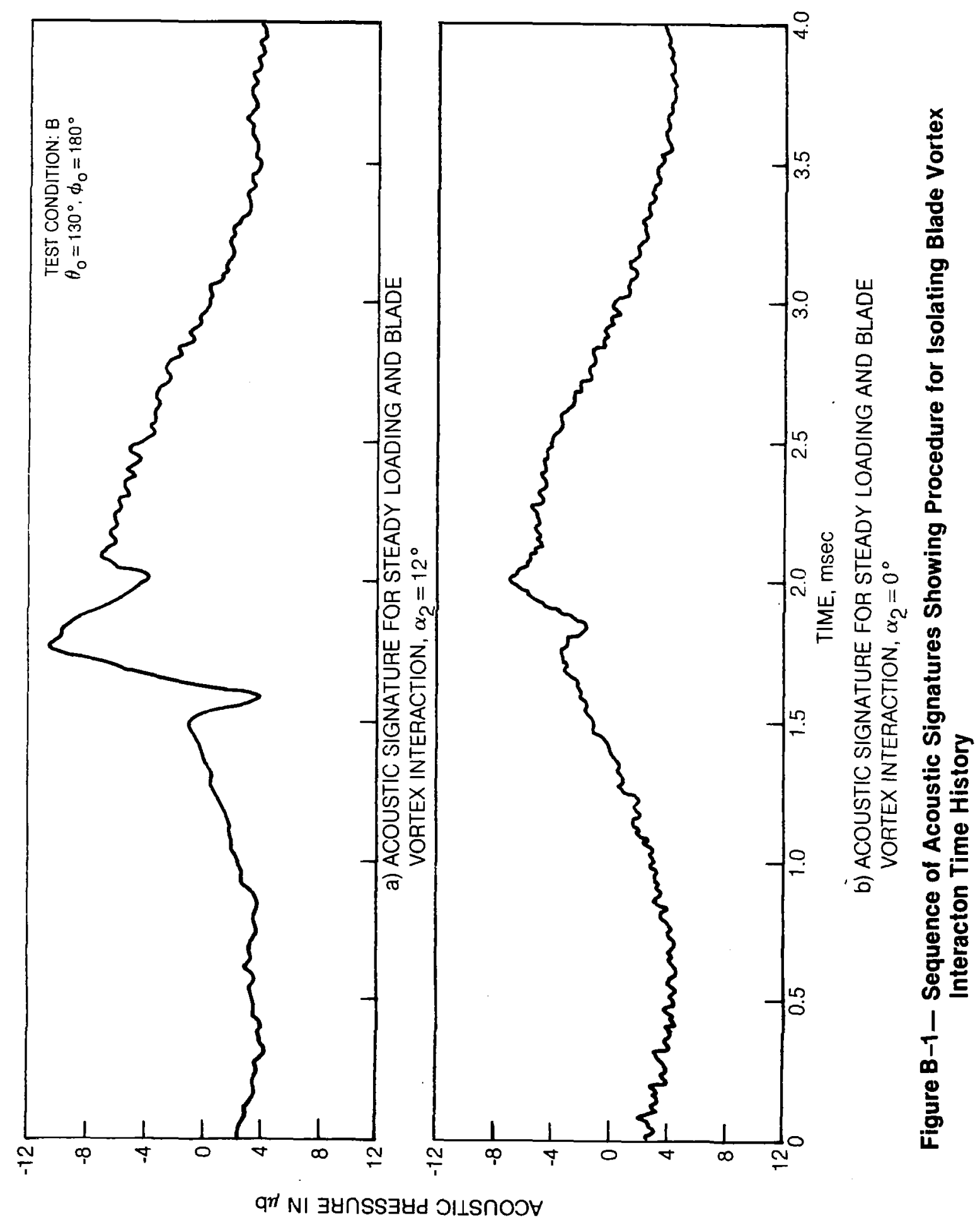




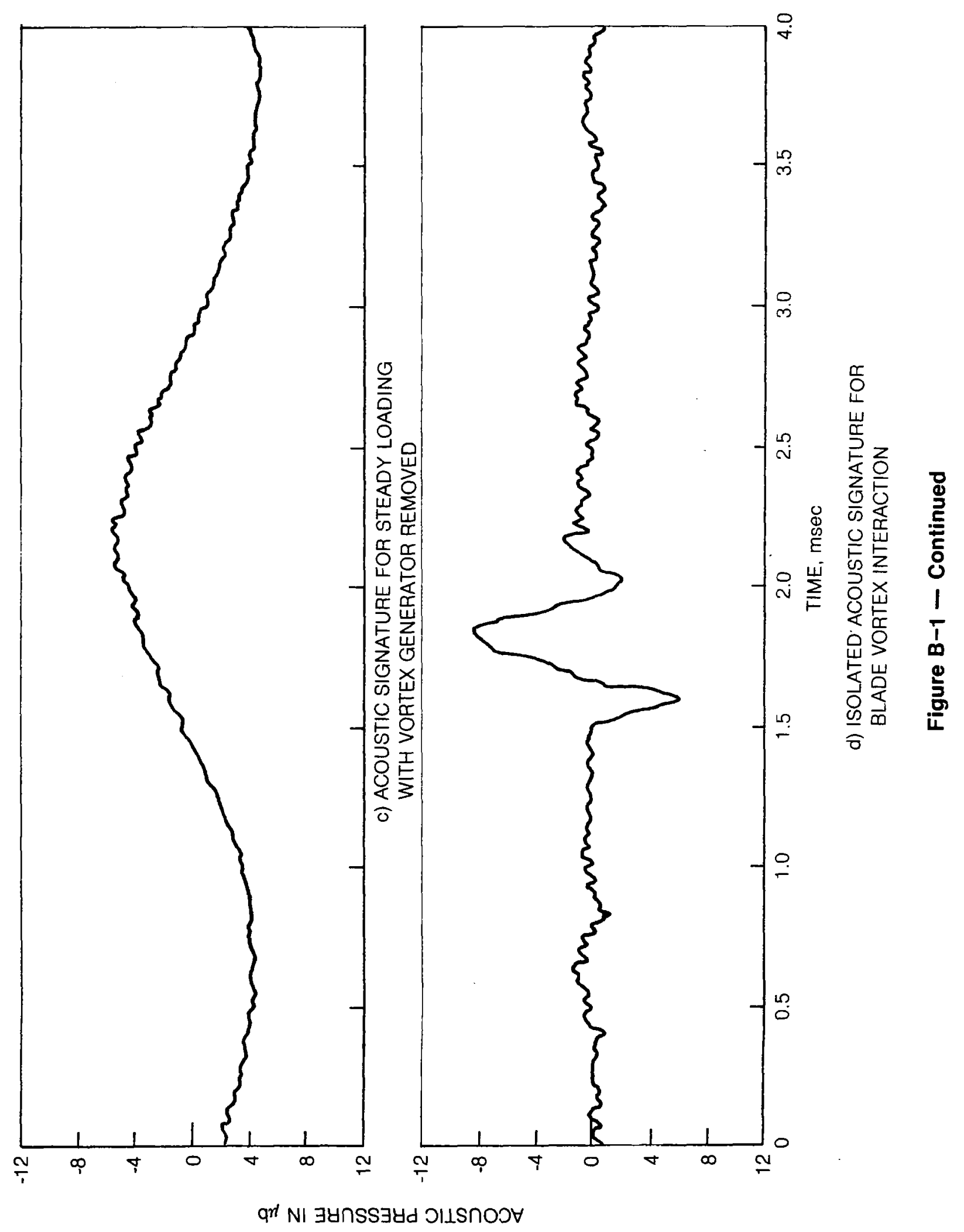




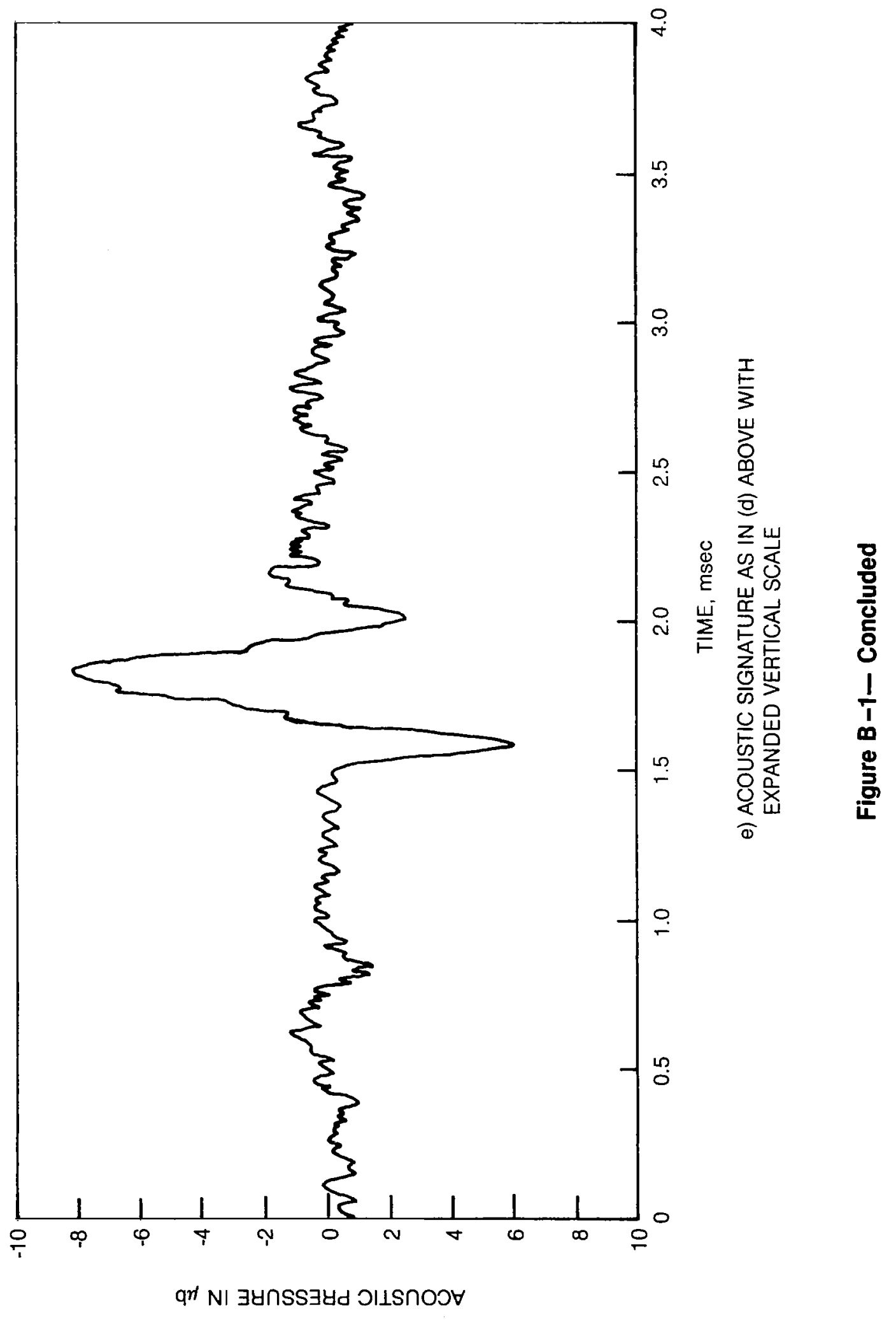




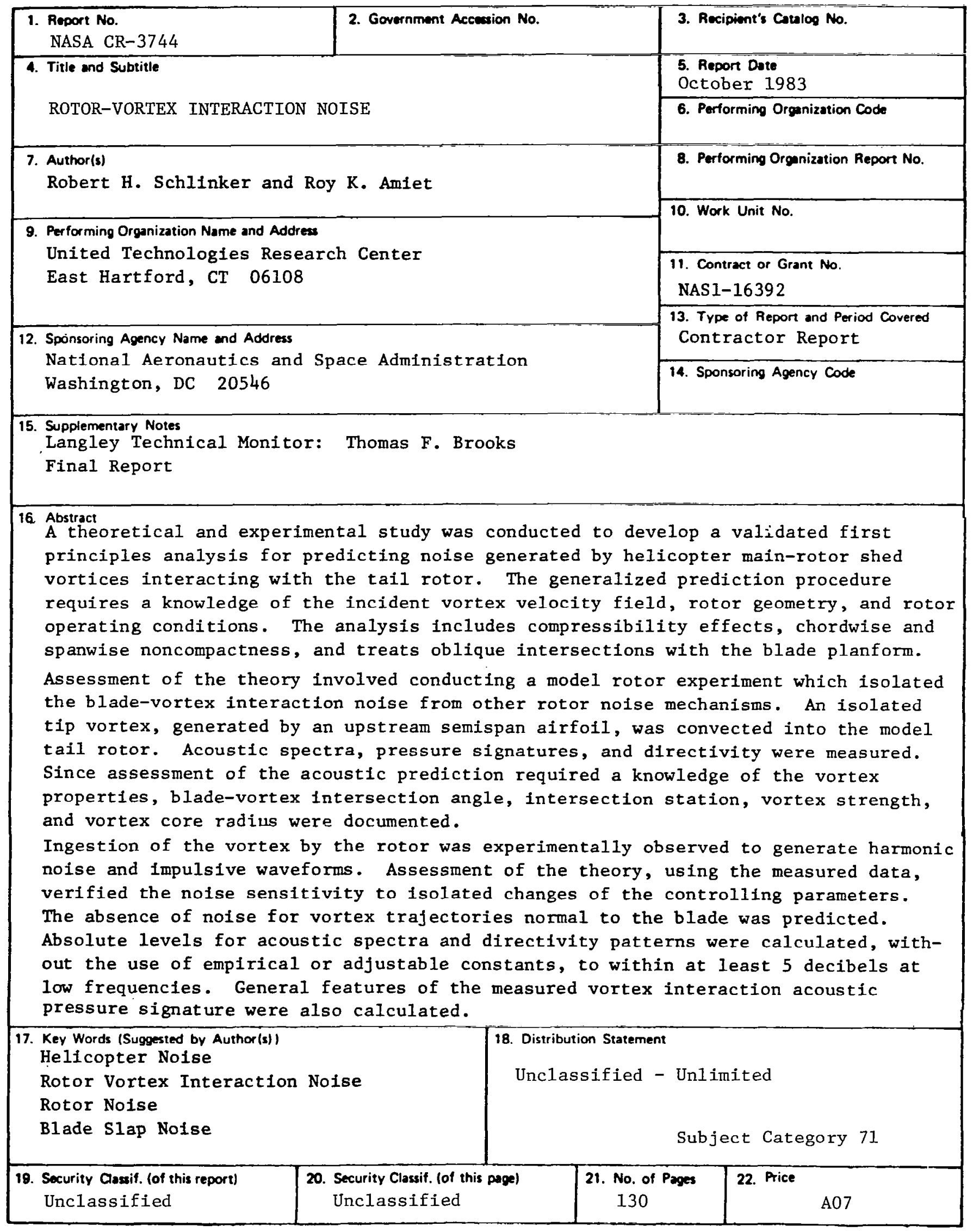

